\title{
Optimal User Authentication Schemes in Mobile Ad hoc Networks
}

\author{
by \\ Jie Liu
}

A thesis submitted to the Faculty of Graduate Studies and Research in partial fulfillment to the requirements for the degree of

\author{
Master of Applied Science
}

Ottawa-Carleton Institute for Electrical and Computer Engineering Department of Systems and Computer Engineering

Carleton University

Ottawa, Ontario, Canada

September 2007

(C) $2007 \mathrm{Jie} \mathrm{Liu}$ 


$\begin{array}{ll}\begin{array}{l}\text { Library and } \\ \text { Archives Canada }\end{array} & \begin{array}{l}\text { Bibliothèque et } \\ \text { Archives Canada }\end{array} \\ \begin{array}{l}\text { Published Heritage } \\ \text { Branch }\end{array} & \begin{array}{l}\text { Direction du } \\ \text { Patrimoine de l'édition }\end{array} \\ \begin{array}{l}\text { 395 Wellington Street } \\ \text { Ottawa ON K1A ON4 }\end{array} & \begin{array}{l}\text { 395, rue Wellington } \\ \text { Ottawa ON K1A ON4 } \\ \text { Canada }\end{array}\end{array}$

Your file Votre référence ISBN: 978-0-494-33658-8 Our file Notre référence ISBN: $978-0-494-33658-8$

NOTICE:

The author has granted a nonexclusive license allowing Library and Archives Canada to reproduce, publish, archive, preserve, conserve, communicate to the public by telecommunication or on the Internet, loan, distribute and sell theses worldwide, for commercial or noncommercial purposes, in microform, paper, electronic and/or any other formats.

The author retains copyright ownership and moral rights in this thesis. Neither the thesis nor substantial extracts from it may be printed or otherwise reproduced without the author's permission.
AVIS:

L'auteur a accordé une licence non exclusive permettant à la Bibliothèque et Archives Canada de reproduire, publier, archiver, sauvegarder, conserver, transmettre au public par télécommunication ou par l'Internet, prêter, distribuer et vendre des thèses partout dans le monde, à des fins commerciales ou autres, sur support microforme, papier, électronique et/ou autres formats.

L'auteur conserve la propriété du droit d'auteur et des droits moraux qui protège cette thèse. $\mathrm{Ni}$ la thèse ni des extraits substantiels de celle-ci ne doivent être imprimés ou autrement reproduits sans son autorisation.
In compliance with the Canadian

Privacy Act some supporting forms may have been removed from this thesis.

While these forms may be included in the document page count, their removal does not represent any loss of content from the thesis.
Conformément à la loi canadienne sur la protection de la vie privée, quelques formulaires secondaires ont été enlevés de cette thèse.

Bien que ces formulaires aient inclus dans la pagination, il n'y aura aucun contenu manquant.

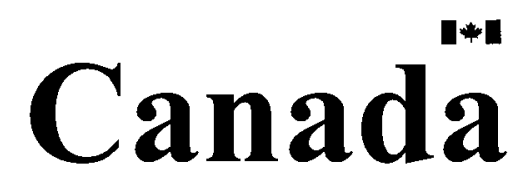




\section{Abstract}

In mobile ad hoc networks (MANETs), continuous user-to-device authentication is desirable so that a system can be monitored for the duration of the session to reduce the vulnerability. This thesis presents an optimal biometric-based continuous authentication scheme in MANETs considering both system security requirements and resource constraints. The thesis formulates the continuous authentication process as a partially observed Markov decision model scheduling algorithms to derive the optimal scheme for continuous authentication. Furthermore, in order to guarantee the network applications and resources securely accessed, the decentralized user-to-network authentication is necessary in MANETs. In this thesis, the decentralized authentication server scheduling problem is modeled as a partially observed Markov decision process with a multi-armed bandit structure. By taking advantage of the information from the intrusion detection system, near optimal server scheduling algorithms are

developed for controlling the information emission to minimize the threat posted to all the servers. 


\section{Acknowledgements}

I would like to thank the invaluable supervision and support of my supervisors Professor Chung-Horng Lung and Professor Fei Richard Yu during the development of this work.

To my family in China, their constant support and love through the course of my studies took me where I am.

My wholehearted thanks go to to my friends and colleagues in the group for their support and help answering my questions.

Specially, to my beloved. 


\section{Contents}

List of Figures

vi

List of Abbreviations

List of Symbols $\quad$ ix

1 Introduction $\quad 1$

1.1 Overview and Motivation . . . . . . . . . . . . . . 1

1.2 Thesis Contributions ....................... 3

1.3 Thesis Structure . . . . . . . . . . . . . . . 5

2 Research Background 6

2.1 Mobile Ad hoc Networks . . . . . . . . . . . . . 6

2.1.1 Self-organization of MANETs . . . . . . . . . . . . 6

2.1.2 Security and Constraints in MANETs . . . . . . . . . 7

2.1.3 Authentication and Continuous Authentication ...... 8

2.2 Biometric-based Authentication . . . . . . . . . . . 9

2.2.1 Characteristics of Biometrics . . . . . . . . . . . 9

2.2 .2 Multimodal Biometrics . . . . . . . . . . . . . 11

2.3 Intrusion Detection Systems . . . . . . . . . . . . . . 14

2.4 Related Work . . . . . . . . . . . . . . . . . . 17 
2.4.1 Some Work in Biometric-based Continuous Authentication . . 17

2.4.2 Some Work in Authentication for Ad hoc Networks . . . . . 18

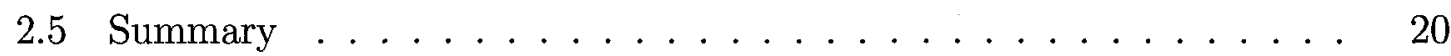

3 MDP and POMDP 21

3.1 Markov Decision Process . . . . . . . . . . . . . . 21

3.1 .1 MDP Model . . . . . . . . . . . . . . . 21

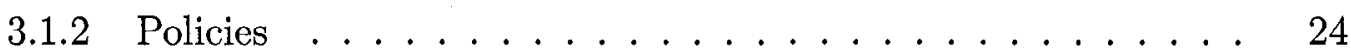

3.1.3 Optimality Criteria . . . . . . . . . . . . 25

3.1 .4 Value Functions . . . . . . . . . . . . 26

3.2 Partially Observable Markov Decision Process . . . . . . . . . . . . 27

3.2 .1 POMDP Model . . . . . . . . . . . . . . 27

3.2 .2 Information State . . . . . . . . . . . . 30

3.2.3 Policies and Value Function of POMDP . . . . . . . . 32

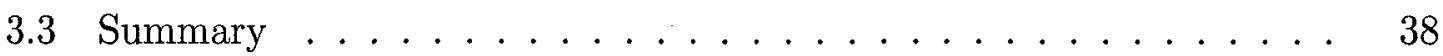

4 Proposed Authentication Schemes $\quad 39$

4.1 Continuous User-to-device Authentication . . . . . . . . . . . 39

4.1.1 Necessity of User-to-device Authentication in MANETs . . . . 39

4.1 .2 System Model . . . . . . . . . . . . . . . 40

4.1 .3 Information State . . . . . . . . . . . . . . . 42

4.1.4 Sensor Scheduling Architecture . . . . . . . . . . . 43

4.1 .5 Cost Definition . . . . . . . . . . . . . . 44

4.1.6 Solving Continuous Authentication Problem . . . . . . . . 46

4.1.7 Scheduling Algorithm with Security Requirement Constraint . 51

4.1.8 Scheduling Algorithm with System Resource Constraint . . . . 53

4.2 User to Network Authentication . . . . . . . . . . . . 55 
4.2.1 Decentralized Authentication Scheme . . . . . . . . . . 55

4.2.2 Authentication Server Scheduling Formulation . . . . . . . 58

4.2.3 Multi-armed Bandit Problem . . . . . . . . . . . . . . 59

4.2.4 Value Iteration Algorithm for Computing Gittins Index . . . . 62

4.2.5 Finite Dimensional Characterization of Gittins Index . . . . 63

4.2.6 Solving the Authentication Server Scheduling Problem . . . 67

4.3 Summary . . . . . . . . . . . . . . . . . . . . . . 69

5 Numerical Examples and Simulation Results $\quad 71$

5.1 Scenario 1 - Optimal Continuous Authentication . . . . . . . . . 72

5.1 .1 Parameters . . . . . . . . . . . . . . . 72

5.1 .2 Results . . . . . . . . . . . . . . . . . . . 74

5.2 Scenario 2 - Multi-modal Biometric Continuous Authentication . . . . 87

5.2 .1 Parameters . . . . . . . . . . . . . . . . 87

5.2 .2 Results . . . . . . . . . . . . . . . . . . . . 89

5.3 Scenario 3 - Multi-server Scheduling . . . . . . . . . . . . . . . . 89

5.3 .1 Parameters . . . . . . . . . . . . . . . . 9 90

5.3 .2 Results . . . . . . . . . . . . . . . . . . . 91

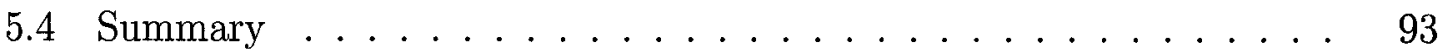

6 Conclusions and Future Work $\quad 94$

$\begin{array}{ll}\text { References } & 97\end{array}$

$\begin{array}{ll}\text { Appendix A: MATLAB Programs } & 101\end{array}$

$\begin{array}{ll}\text { Appendix B: POMDP Files } & 109\end{array}$ 


\section{List of Figures}

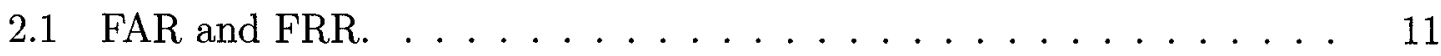

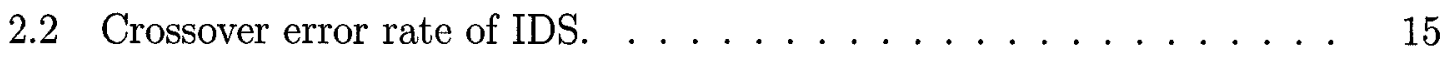

3.1 Markov decision process. . . . . . . . . . . . . . . . 22

3.2 Relationship between time and dynamic programming indices $\ldots .26$

3.3 POMDP model. . . . . . . . . . . . . . . . . . . . . . . . 28

3.4 Information space with 2 states and 3 states. . . . . . . . . . . 29

3.5 POMDP dynamic model. . . . . . . . . . . . . . . . . . . 31

3.6 Value function over information space. . . . . . . . . . . . 34

3.7 PWLC value function. . . . . . . . . . . . . . . . . . 35

4.1 Sensor scheduling. . . . . . . . . . . . . . . . . . . 40

4.2 HMM sensor scheduling and information state update. . . . . . . 43

4.3 Estimation error and its upper bound, lower bound approximations. . 48

4.4 User authentication procedures. . . . . . . . . . . . . 56

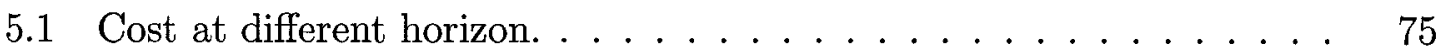

5.2 Cost over infinite horizon with approximation points $\mathrm{R}=36 \ldots \ldots 76$

5.3 Cost over infinite horizon with approximation points $\mathrm{R}=64 \ldots \ldots 76$

5.4 Policy over information state with FAR=FRR=0.1 $\ldots \ldots \ldots \ldots 77$ 
5.5 Sensor using over information space, $\mathrm{p}=1-\mathrm{FAR}=1-\mathrm{FRR} . \quad \ldots \quad . \quad 79$

5.6 Using iris biosensor over 30 horizons with different transition probability. 80

5.7 Sensors usage over information space with and without constraints (top line - without constraints; bottom line - with constraints). . . . . . 81

5.8 Times of using iris biosensor when $\mathrm{p}=1-\mathrm{FRR}=1-\mathrm{FAR}=0.8 . \ldots 86$

5.9 Times of using iris biosensor when $\mathrm{p}=1-\mathrm{FRR}=1-\mathrm{FAR}=0.9 . \quad . \quad 86$

5.10 Sensors usage over information space. . . . . . . . . . . . . 89

5.11 Gittins indices for two types of servers. . . . . . . . . . . . 92

A-1 Matlab file for computing result $1 \ldots \ldots$. . . . . . . . 101

A-2 Matlab file for computing result 2 page $1 \ldots \ldots$. . . . . . . . . 102

A-3 Matlab file for computing result 2 page $2 \ldots \ldots . \ldots 103$

A-4 Matlab file for computing result $3 \ldots$. . . . . . . . . . . . 104

A-5 Matlab file for computing result 4 page $1 \ldots \ldots$. . . . . . . . 105

A-6 Matlab file for computing result 4 page $2 \ldots \ldots$. . . . . . . 106

A-7 Matlab file for computing result 4 page $3 \ldots \ldots$. . . . . . . . 107

A-8 Gittins indices for Server type 1 and Server type $2 \ldots \ldots$

B-1 POMDP configuration file of Scenario 2 . . . . . . . . . . . 109

B-2 POMDP configuration file of Server type $1 \ldots \ldots$. . . . . . . 110

B-3 POMDP configuration file of Server type $2 \ldots \ldots \ldots 111$ 


\section{List of Abbreviations}

$\begin{array}{ll}\text { CER } & \text { Crossover Error Rate } \\ \text { CO-MDP } & \text { Completely Observable Markov Decision Process } \\ \text { DBN } & \text { Dynamic Bayesian Network } \\ \text { DoS } & \text { Deny of Service } \\ \text { EER } & \text { Equal Error Rate } \\ \text { FAR } & \text { False Acceptance Rate } \\ \text { FNR } & \text { False Negative Rate } \\ \text { FPR } & \text { False Positive Rate } \\ \text { FRR } & \text { False Rejection Rate } \\ \text { GSM } & \text { Global System for Mobile communications } \\ \text { HMM } & \text { Hidden Markov Model } \\ \text { IDS } & \text { Intrusion Detection System } \\ \text { MANET } & \text { Mobile Ad hoc Network } \\ \text { MDP } & \text { Markov Decision Process } \\ \text { PDA } & \text { Personal Digital Assistant } \\ \text { PIN } & \text { Personal Identification Number } \\ \text { PWLC } & \text { Piecewise Linear and Convex } \\ \text { POMDP } & \text { Partially Observed Markov Decision Process } \\ \text { SIM } & \text { Subscriber Identity Module } \\ & \end{array}$




\section{List of Symbols}

page where

symbol

Symbol

Explanation

is defined

A

$a_{i}$

$a^{k}$

$B$

$\beta$

$b_{j \theta}^{a}$

$\chi(l)$

$c^{k}\left(X^{k}, l\right)$

$D$

$\delta$

$\delta_{k}$

$e_{i}$

F

$\gamma(l, x(l))$
System action space

Action

The biosensor selected at time $k$ 41

Observation Matrix

Discount factor

The probability that $\theta$ was observed when the system state is $s_{j}$ and last action taken at time $k-1$ was $a \ldots \ldots \ldots \ldots . . \ldots 28$

State space of information states $x^{k}(l)$ see (4.35)

60

Instantaneous cost of using the sensor $l$ when the device state is $X^{k}$

Quantized norm

Stationary policy

Non-stationary policy at time $k$

$S$-dimensional unit vector with 1 in the $i t h$ position and zeros elsewhere

The set of information state 
$\Gamma^{k} \quad$ Finite set of vectors $\gamma_{i}^{k}$ see $(4.12) \quad \ldots \ldots \ldots \ldots \ldots \ldots \ldots \ldots \ldots \ldots$

$\gamma_{i}^{k} \quad S$-dimensional vector see $(4.12) \quad \ldots \ldots \ldots \ldots \ldots \ldots \ldots \ldots \ldots \ldots$

$g\left(\pi^{k}\right) \quad S$-dimensional estimation error vector see $(4.10) \ldots \ldots \ldots \ldots .45$

$H \quad$ Number of linear segments $\ldots \ldots \ldots \ldots \ldots \ldots \ldots \ldots \ldots \ldots \ldots \ldots \ldots$

$J \quad$ Cumulated cost used in value function see $(4.8) \ldots \ldots \ldots \ldots$

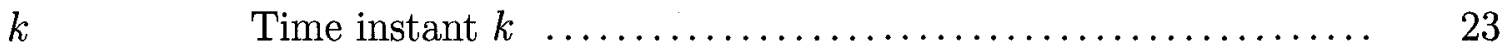

Number of biosensors $\quad \ldots \ldots \ldots \ldots \ldots \ldots \ldots \ldots \ldots \ldots \ldots \ldots \ldots$

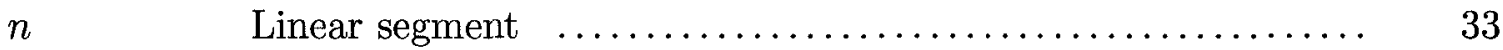

$b_{i}\left(a^{k}, O_{m}\left(a^{k}\right)\right)$ The probability of observation $m$ acquired when biosensor $a^{k}$ is picked at time $k$ given the state $i$ of Markov chain, see (4.1) 41

$O_{M_{l}}(l) \quad$ The $M_{l}$ th observation symbol for biosensor $l \ldots \ldots \ldots \ldots \ldots .41$

$P \quad$ System probability transition matrix $\ldots \ldots \ldots \ldots \ldots \ldots \ldots \ldots$

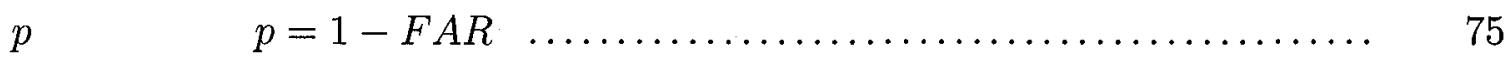

$\pi_{i} \quad$ The probability that system stays in state $s_{i} \ldots \ldots \ldots \ldots \ldots$

$p_{i j} \quad$ Transition probability from state $i$ to state $j \ldots \ldots \ldots \ldots \ldots .23$

$Q(\cdot) \quad$ Transition probability matrix see $(4.30) \quad \ldots \ldots \ldots \ldots \ldots \ldots \ldots$

$q_{i}^{a} \quad$ Expected reward when current system state is $i$ and action $a$ is

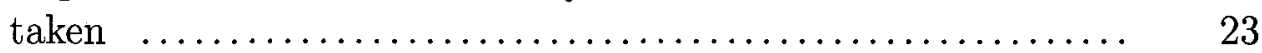

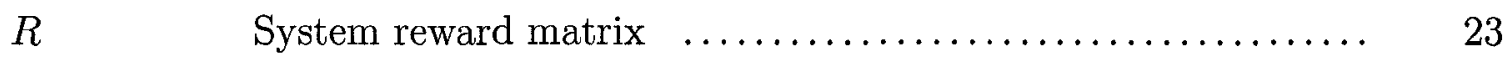

$r_{i j}^{a} \quad$ Immediate reward when system moves from state $i$ to state $j$ while action $a$ is performed $\ldots \ldots \ldots \ldots \ldots \ldots \ldots \ldots \ldots, 23$

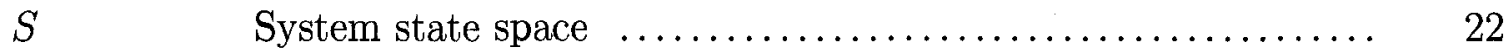

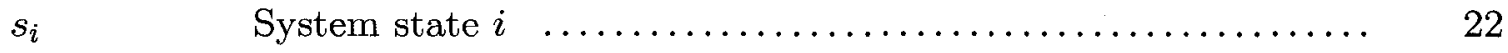

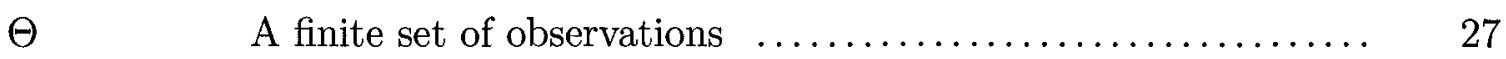

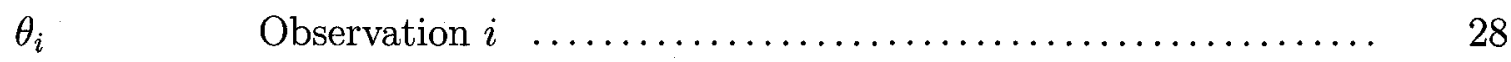

$u^{k}(l) \quad$ Positive scalar weight for biosensor $l$ at time $k \ldots \ldots \ldots \ldots$ 
$V_{k}^{\delta^{k}}(s) \quad$ Value acquired when system start in state state $s$ and execute the policy $\delta^{k}$ for $K-k$ time steps $\ldots \ldots \ldots \ldots \ldots \ldots \ldots \ldots$

$X^{k} \quad S$-state first order Markov chain $\ldots \ldots \ldots \ldots \ldots \ldots \ldots \ldots \ldots$

$x^{k}(l) \quad$ Information state at time $k$ for server $l$ see $(4.34) \ldots \ldots \ldots \ldots$

$Y^{k} \quad$ Information available at time $k \quad \ldots \ldots \ldots \ldots \ldots \ldots \ldots \ldots \ldots \ldots \ldots \ldots \ldots \ldots$

$y^{k}\left(a^{k}\right) \quad$ The observation of biosensor $a^{k} \quad \ldots \ldots \ldots \ldots \ldots \ldots \ldots \ldots \ldots \ldots \ldots$ 


\section{Chapter 1}

\section{Introduction}

\subsection{Overview and Motivation}

Mobile ad hoc networks (MANETs) enable wireless devices to dynamically establish networks without necessarily using a fixed infrastructure. Wireless nodes rely on another to keep a network connected. MANETs introduce different security risks from those for fixed networks due to system constraints in mobile devices and the dynamic nature of ad hoc networks. Although there are many security issues in MANETs, user authentication is the core requirement for integrity, confidentiality and non-repudiation [1]. Authentication is the process of confirming the identity claimed by a user and ensure the resources accessed by an authentic user. Authentication can be performed by using one or more of the validation factors: what you know, such as a password; what you have, such as a token; and what you are, a user's biometrics, such as a fingerprint [2]. For the password, it is simple and easy to use, but difficult to distinguish an authentic user from impostors since there is no direct connection between a user and a password. For the token, in addition to no connection between a user and a token, it is easy to be lost or be counterfeited. Biometrics is the only 
way to have direct connection with the identity of the user. It has been implemented in many security related systems, including MANETs [2].

Most systems work by authenticating a user during login and then operate under the assumption that the system is secure from that time forward. However, in a hostile environment where chances of node being captured are relatively high, continuous authentication, or re-authentication is desirable so that a system can be monitored for the duration of the session to reduce the vulnerability [3].

There are two classes of user authentications in MANETs: user-to-device authentication and user-to-network authentication. When biometric-based authentication is applied to MANETs, the main difference between the above two authentications is where the user's biometric templates are stored: in the device or in the network servers. Since there is no centralized servers available in MANETs, distributed authentication schemes are desirable in MANETs.

The experience in security of wireline networks indicates the importance of multilevel protections because there are always some weak points in the system, no matter what is used for authentication. This is especially true for MANETs, given the low physical security of mobile devices. To solve this problem, intrusion detection systems (IDS), serving as the second wall of protection, can effectively help identify malicious activities. An IDS continuously or periodically monitors the current subject activities, compares them with stored normal profiles and/or attack signatures, and initiates proper responses [4].

Although much work has been conducted to address continuous authentication, there is few report in the literature about how to optimally schedule different biometrics taking into account of the system security requirements and resource constraints in MANETs. Some examples of constraints include limited battery power, low-power microprocessor, low bandwidth and small memory. To the best of our knowledge, 
design of optimal biometric-based continuous authentication schemes that consider system security requirements and resource constraints in MANETs has not been addressed in previous work.

There are different device-to-network authentication strategies discussed for MANETs [1][2][5]. However, there are few authentication schemes that work jointly with the IDS and share information with each other as to obtain more efficient and threatless mechanisms. An ideal user-to-network authentication scheme used in MANETs should be fully decentralized, easily to be scalable, computationally intractable, and with minimum communication overhead.

Therefore, the motivation of the thesis is to develop optimal schemes covering both user-to-device and user-to-network authentications in MANETs: An optimal biometric-based approach that is used for continuous user-to-device authentication considering the resource constraints and security constraints; an efficient distributed approach that combines IDS with the user-to-network authentication.

\subsection{Thesis Contributions}

This thesis deals with user authentication in MANETs. The main contributions from this work to the field are that two optimal authentication schemes are proposed:

1. An optimal biometric-based continuous user-to-device authentication scheme considering both the system security requirements and resource constraints.

2. A decentralized user-to-network authentication scheme considering the threat level to the servers.

In scheme 1, with the linear approximation of estimation error incurred by biosensors, the continuous authentication problem is modeled as a partially observed Markov 
decision process (POMDP). Optimal dynamic programming-based hidden Markov model (HMM) scheduling algorithms are presented. Some distinct features of the proposed scheme are as follows:

- It can optimally control whether or not to perform authentication as well as which biometrics to use to minimize the usage of system resources.

- The scheme can dynamically adapt to the system security status, and select the optimal biometrics.

- The scheme performs authentication continuously by integrating the information across modalities in serial mode.

In scheme 2 , the decentralized authentication server scheduling problem is modeled as a partially observed Markov decision process with a multi-armed bandit structure, a near optimal server scheduling algorithm has been developed for controlling the information emission to minimize the threat posted to all the servers. The main features of this scheme are as follows:

- It can dynamically decide which server will act as the authentication server according to the information emission and hence it decreases the threat level in MANETs.

- Being modeled into multi-armed bandit structure, the optimal server scheduling problem is indexable with Gittins index rule. That is, the server with the highest Gittins index will act as the authentication server and the algorithm is fully decentralized.

- By using the Gittins index rule, our scheme simplifies multi-server POMDP problem into a finite number of single-server optimization problem. Therefore, 
the server scheduling problem is scalable and the algorithm is suitable for largescale networks.

A paper based on part of this work has been accepted in the proceeding of the IEEE Wireless and Mobile Computing, Networking and Communications (WiMob) conference:

- J. Liu, F. R. Yu, C. H. Lung and H. Tang, "Optimal Biometric-Based Continuous Authentication in Mobile Ad hoc Networks", to appear in Proc. IEEE Wireless and Mobile Computing, Networking and Communications, White Plains, New York, Oct. 2007.

Another paper based on part of this work was submitted to Infocom 2008:

- J. Liu, F. R. Yu, C.-H. Lung and H. Tang, "Optimal Combined Intrusion Detection and Biometrics-Based Continuous Authentication in High Security Mobile Ad hoc Networks", submitted to INFOCOMM'08, Phoenix, Arizona.

\subsection{Thesis Structure}

The structure of this thesis is as follows. Chapter 2 presents the background for this thesis, and summarizes the state-of-the-art in the user authentication in MANETs. Chapter 3 describes the POMDP problem and solving algorithms. The policy and value function of POMDP are also described in this chapter. The novel user-to-device and user-to-network authentication schemes are illustrated in Chapter 4. Chapter 5 shows the numerical examples of above two schemes. Finally, Chapter 6 summarizes this thesis's contribution and provides further work directions. 


\section{Chapter 2}

\section{Research Background}

\subsection{Mobile Ad hoc Networks}

In recent years, MANETs have become a popular subject because of their selfconfiguration and self-organization capabilities. Wireless nodes can establish a dynamic network without the need of a fixed infrastructure. A node can function both as a network router for routing packets from the other nodes and as a network host for transmitting and receiving data. MANETs are particular useful when a reliable fixed or mobile infrastructure is not available. Instant conferences between notebook PC users, military applications, emergency operations, and other secure-sensitive operations are important applications of MANETs due to their quick and easy deployment.

\subsubsection{Self-organization of MANETs}

Due to the complete lack of centralized control, MANTETs nodes cooperate with each other to achieve a common goal. The major activities involved in self-organization are neighbor discovery, topology organization, and topology reorganization. Through periodically transmitting beacon packets, or promiscuous snooping on the channels, the 
activities of neighbors can be acquired. Each node in MANETs maintains the topology of the network by gathering the local or entire network information. MANETs need to update the topology information whenever the networks change such as participation of new node, failure of node and links, etc. Therefore, self-organization is a continuous process that has to adapt to a variety of changes or failures.

\subsubsection{Security and Constraints in MANETs}

The security in MANETs is very important especially in military environments. Unlike the wireline networks, MANETs are inherently insecure because of the lack of any central authority and shared wireless medium. The major security threats that exist in ad hoc wireless networks are as follows: denial of service, resource consumption, host impersonation, information disclosure, and interference. The unique characteristics of MANETs present some new challenges to security design [6].

- Shared wireless broadcast radio: A node can receive and transmit data from and to all the nodes within its direct transmission range.

- Insecure operation environment: MANETs may operate in hostile environments, especially for the tactical MANETs. Nodes frequently move in and out of hostile enemy territory. The chances of node capture are high in such environments, which requires re-authentication.

- Lack of central coordination: There is no centralized network management functionality in MANETs. The existing security solutions for wired networks cannot be applied directly to the MANETs domain.

- Lack of association: Because of the dynamic characteristic of MANETs, it is difficult to find a proper authentication mechanism to use for associating nodes with a network. 
- Limited resource availability: Bandwidth, battery power, and computational power are scarce in MANETs.

In tactical MANETs, there are some extra requirements for security design:

- High security requirement: Since MANETs need to transmit some critical information, security is paramount important.

- User multitasking: In battle fields, user intervention should be minimized.

\subsubsection{Authentication and Continuous Authentication}

A complete security scheme should encompass all three security components of prevention, detection, and reaction [7]. As the front line of defense, user authentication is crucial for integrity, confidentiality and non-repudiation [1]. Authentication is the process of confirming the identity claimed by a user and ensure the resources accessed by an authentic user. Authentication can be performed by using one or more of the validation factors [8]: (i) Something a user knows, which is very useful for small devices, such as a password and personal identification number (PIN); (ii) Something a user has, such as a electrical token and subscriber identity module (SIM) cards in global system for mobile communications (GSM); (iii) Something a user is, it is known as biometric authentication, such as a fingerprint [2]. For the password, it is simple and easy to use, but difficult to distinguish an authentic user from impostors since there is no direct connection between a user and a password. For the token, in addition to no connection between a user and a token, it is subject to be lost or be counterfeited. Biometrics is the only way to have direct connection with the identity of the user.

We distinguish two classes of user authentications in MANETs: user-to-device and user-to-network. A user has to authenticate himself/herself to the device before 
he/she can use it. User-to-network authentication denotes that user needs to be authenticated to the network before it communicates with the other users and use the wireless applications. In tactical MANETs, user and device are generally tightly coupled. If the user-to-device authentication fails, it means that the device is not in the right hand and appropriate measures should be taken accordingly. Therefore, attacks such as reading or modifying data stored in the device or further accessing to network resource can be prevented.

By convention, most authentication systems do not usually require the user to do re-authentication for continued access to the protected resources [3]. However, for MANETs that work in hostile environment, the rate of node capture is high and it is critical to track the user continuously during the lifetime of a node. In other words, authentication is not merely used to authenticate a user at the initial login, but that it is used continuously to verify the presence of the authentic user. The frequency of re-authentication depends on the severity of the situation in which the MANET has been established and the resource constraints of the network.

\subsection{Biometric-based Authentication}

Biometrics is a technique commonly known as the automatic identification or verification of an individual by his or her physiological or behavioral characteristics [2]. Biometrics provides some possible solutions to authentication used in MANETs, since it has direct connection with user identity and needs little user interruption [2] [9].

\subsubsection{Characteristics of Biometrics}

Biometric systems make use of fingerprints, hand geometry, iris, face, retina, facial thermograms, signature or voiceprint to verify a person's identity. They have an 
edge over traditional security methods in that they cannot be easily shared or stolen. Some differences of biometrics from traditional authentication need to be considered as follows [10]:

- Biometrics is not completely accurate: For a password, it can be encrypted and compared on a byte-by-byte basis with the encrypted password stored in the system password's database, and the results of the comparison is a boolean: true or false. The results of token-based authentication are presented in the same way: with token, the result is true, or else is false. But for the biometrics, because of normal variations in measured features and measurement environments, the result of comparison is a closeness of match. So the access control portion of a biometric authentication system must be set to accept the identity of users at some threshold.

False rejection rate (FRR) denotes the fraction of the number of rejected client patterns divided by the total number of client patterns; false acceptance rate (FAR) denotes the fraction of the falsely accepted patterns divided by the number of all impostors. Both of them are used to describe the accuracy of biometric system [11]. The relationship between FAR and FRR is illustrated in the Figure 2.1. In the left part of Figure 2.1, the score is used to express the similarity between a patten and a biometric template. The higher the score, the higher the similarity between them. Theoretically speaking, the client scores should always be higher than the scores of impostors. But this is not always true since the biometrics is not always $100 \%$ correct. Therefore, if the clients scores and impostors scores are overlap, the selection of threshold will become a problem. In the right part of Figure 2.1 [11], the FAR and FRR intersect at a point, here $F A R=F R R$, and we call this point as Equal error rate (EER). 

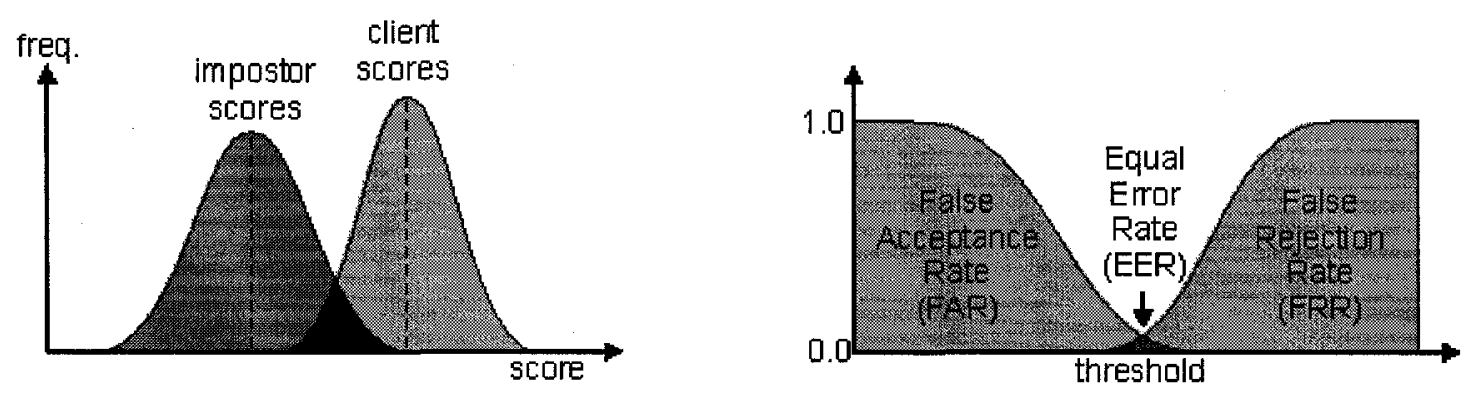

Fig. 2.1 FAR and FRR.

- Biometrics can be continuously monitored: When a user is conducting his or her activities, some biometrics can be continuously monitored without causing disruption of normal activities. For example, the user's face image can be collected while the user is working before a monitor. The continuous result of authentication is helpful to handle failed authentication attempts. Since the continuous biometric system will occasionally produce failed user authentication checks, the decision-maker of biometric system should make their decision based on a history of results rather than just one reading.

- Biometrics is expensive to compute: Normally, the computation and comparison of biometrics require much more computation than password or token verification. This concern is more substantial in continuous authentication. Hence, the computational costs must be addressed for re-authentication with biometrics in MANETs.

\subsubsection{Multimodal Biometrics}

Each biometric technology has its own strengths and weaknesses. For example, some technologies can provide high accuracy, but may be expensive or difficult to use; signature is a widely accepted authentication method, but it still remains a question if 
it could acquire the same level accuracy as the other biometric technologies. Currently, there is no best biometric modality since it depends on the environment applied.

Unimodal biometrics has to face several challenges such as noise in sensed data, intra-class variations, inter-class similarities, non-universality, spoof attacks, etc [12]. Some of these problems could be resolved by adopting multimodal biometric systems. Multimodal biometric system presents a more reliable authentication method due to the combination of statistically independent biometric traits [13]. This system can exploit the benefits of one biometric and mitigate the shortcomings of another biometric. Furthermore, randomly selecting a subset of biometric traits further ensures that the authentic user is presented.

Multimodal biometric systems help achieve an increase in performance over that only using a single biometric. However, finding an effective fusion system is necessary to combine the information presented by multiple biosensors.

A general biometric system has four important components:

- Sensor module which acquires the biometric data of a user.

- Feature extraction module which processes the data to extract the feature values.

- Matching module which compares the feature values with the template to generate a matching score.

- Decision-making module in which the result of verification or authentication is acquired: accept or reject.

In a multimodal biometric system, the information fusion can occur in any of the aforementioned modules. (i) Fusion at the data or feature level: The data acquired from different sensor can be used to compute a new vector. (ii) Fusion at the match score level: Match scores provided by each system can be combined to minimize 
the FRR for a given FAR. (iii) Fusion at the decision level: The final output of multiple classfiers can be consolidated by some techniques such as via majority voting. Normally, the earlier stage the data is merged, the more effective the system will be. However, fusion at data level or feature level is difficult in practise. Fusion at the decision level is considered to be rigid. Therefore, the score level fusion is usually preferred.

The increasing use of multimodal biometrics has led to the investigation of different modes of system operation: serial mode, parallel mode, and hierarchical mode [12]. In serial mode of operation, one output of a biosensor will be used at one time. Therefore, multimodal biometric traits do not need to be acquired simultaneously, and the decision could be made before all biometric traits are received. Accordingly, the recognition overall time can be reduced, which is important for MANETs. In the parallel mode of operation, multimodal biometric traits have to be used simultaneously. The hierarchical mode of operation is suitable for the system using the large number of biometric traits. This thesis will consider the serial mode of operation since the continuous authentication is necessary for MANETs.

The strategies of biometric-based user authentication are normally classified into as user-to-device or user-to-network authentication, which differs in the storage locations of the biometric templates. For user-to-device authentication, the biometric templates are stored in the device to build a trust between user and devices. In order to provide a user's identity to the existing network, the users' biometric templates are kept in one or some servers in the network [2]. 


\subsection{Intrusion Detection Systems}

By definition, intrusion is any set of actions that attempts to compromise the integrity, confidentiality or availability of resources. MANETs are vulnerable due to its features of open medium, mobility of the nodes, lack of centralized node for monitoring and management, and no prior security association available. Authentication and encryption are common intrusion prevention measures used in MANETs to reduce the intrusions but cannot eliminate them because each intrusion measure has its own weakness. Normally, in MANETs, a malicious node can launch deny of service (DoS) or disrupt the routing mechanism by generating error routing messages. For all these types of attacks, intrusion detection presents a second wall of defense and is of paramount importance in any high-survivability network.

For any intrusion detection system, the basic assumption is that the programs activities should be under observation. Considering the type of data the IDSs use, the IDSs can be categorized into three classes [14]. (i) Network-based intrusion detection: It runs at the gateway of a network and examines all incoming packets. (ii) Host-based intrusion detection: It receives the necessary audit data from the operating system and analyzes the generated events. (iii) Router-based intrusion detection: It is installed on routers to prevent intruder from entering into the network. In MANETs, only the second class IDS can be used since there is no gateway or routers available, and all the possible audit data are any data available during the communication within the radio range. A host-based IDS, which relies on the data generated by the users or programs located on the host, is the choice for MANETs [4].

From the functional characteristics of detection schemes, intrusion detection systems can be classified into three categories [15]:

- Anomaly detection: In a anomaly detection, if an activity differs from the es- 
tablished normal profile, it is treated as a possible intrusion. The advantages of this approach is that it does not require prior knowledge but its disadvantage is with high false positive rate.

- Misuse detection: In a misuse detection system, the patterns of well-known intrusive process and traces are known in advance. The advantages of this approach is that the detection is accurate and its disadvantages is that the system cannot detect some newly invented attacks.

- Specification-based detection: In this approach, a set of constraints are defined for correct operation of a program or protocol. An alarm flag will be raised if there is any deviation from the constraints. This approach could detect some new invented attacks with low false positive rate.

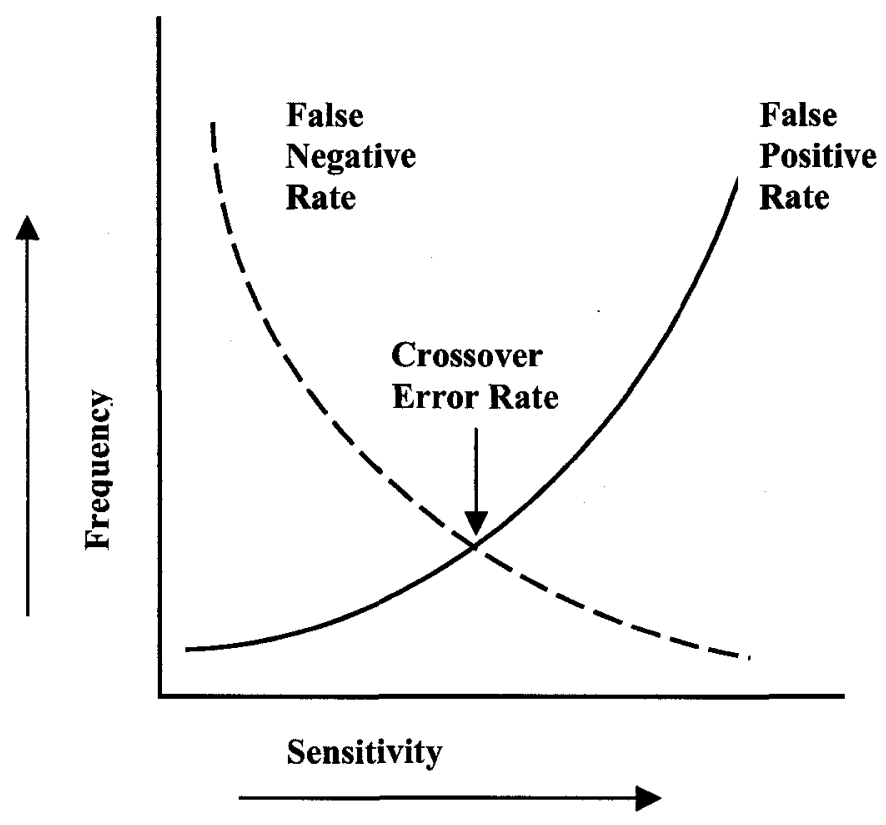

Fig. 2.2 Crossover error rate of IDS.

An IDS can automatically monitor and analyze the events, once the attacks are 
detected, alarms will be generated or the measures will be activated to protect systems. The parameters false positive rate (FPR), false negative rate (FNR), crossover error rate (CER) (Figure $2.2[16]$ ) are often used to provide a baseline measure for comparison of intrusion-detection systems [16].

- False positive rate is the frequency with which the IDS reports malicious activity in error. High FPR will cause the administrator ignore the true alarms. In general, increasing the sensitivity of an IDS results in a higher FPR, while decreasing the sensitivity results in a lower FPR.

- False negative rate is the frequency with which the IDS fails to report a malicious activity. FNR changes inversely to FPR, in other words, as FNR increases, the FPR decreases and vise-versa. FNR is a very dangerous type of errors.

- Crossover error rate is determined by adjusting the system's sensitivity until the PNR equals to PPR.

The selected values of FPR and FNR depend on the system security requirement. Simply selecting the IDS with lowest ECR can bring a balance between false negatives and false positives. Therefore it is reasonable for us to treat an IDS as a sensor that can correctly detect the system state with some percentage that is decided by a threshold. Since we can treat an IDS as a sensor, it is possible to incorporate IDS into our authentication scheme.

Intrusion detection and response systems should work collaboratively to meet the needs of MANETs. A few main responses are [15]:

- Re-initialize communication channels between nodes.

- Reorganize the network to exclude the compromised nodes. 
- IDS informs the end node which may take take some actions.

- Initialize a re-authentication request.

In our proposed decentralized authentication scheme, the nodes will take appropriate actions according to the output of IDS.

\subsection{Related Work}

\subsubsection{Some Work in Biometric-based Continuous Authentication}

Dynamic Bayesian network (DBN) was used in [17] for continuous multimodal authentication to account for classification uncertainty and to encode the system's dynamic model. By modeling more hidden variables, DBNs were capable of modeling important contextual information.

Authors in [3] proposed a continuous multimodal biometrics system using a HMM. In this work, the user's identity was the sole hidden variable that would "emit" biometric measurements. The authors also proposed several new metrics which took the time factor into consideration for multimodal biometrics used in continuous verification. However, there are few reports in the literature about how to optimally schedule different biometrics based on the system security.

Klosterman and Ganger [10] examined the differences between biometrics and traditional passwords/tokens and argued that biometrics is suitable for continuous authentication. They enabled a Linux system to continuously authenticate the user and the action is taken in response to a timer that is triggered periodically. A separate machine is used to make actual authentication decision because of high-computational cost.

In [18], Altinok and Turk applied the face, voice and fingerprint biometrics for 
continuous authentication. They argued that continuous authentication need to integrate the time and modality. The multimodal system weighted each modality at the score level, and the weighting factor was decreased monotonically with the time since the last measurement.

The authors in [9] developed a multimodal authentication system for the secure phone project by using the combination of voice, face, and signature biometrics. The storage and processing of the client's biometric profile all were done on the SIM-card in the PDA and only the service provider can access to the SIM-card. The benchmark databases BANCA (audio-visual) and BIOMET (signature) are used. This paper proved that simple multimodal biometrics authentication scheme can be implemented on a small personal digital assistant (PDA) device.

\subsubsection{Some Work in Authentication for Ad hoc Networks}

Xiao [2] first addressed the needs of biometric authentication for information security in ad hoc networks. Then the advantages and disadvantages of common biometric methods were introduced and compared. A new decentralized authentication model designed for high security, small group coalition operation was proposed in this paper: Soldiers are divided into small groups, and the group leader keeps the group members' biometrics templates and acts as the distributed authentication server. There are different cryptographic keys used within the group and between group leaders.

Weimerskirch presented a survey of various existing models and analyzed them in terms of scope and applications in [1]. The model he proposed deals with trust and security in ad hoc networks and does not require devices with strong processors as public-key systems. In his model, each entity maintains a repository of trustworthy entities. By asking for the references from the distributed trust models, the trust relationships can be established. As long as the number of compromised nodes is 
below a threshold, the authentication result can not be harmed.

In [5], Gokhale proposed a cryptographical secure way of representing trust relationships between the nodes in the network. Secure groups structure, troups, is created in a peer-to-peer manner using RSA accumulators. Each node in a group has an identity within a group, but it does not need to reveal its identity during verification.

Even though each scheme discussed above implemented the authentication or verification in a decentralized way, all the schemes were not combined with the IDS which is an important part in MNAETs. 


\subsection{Summary}

This chapter introduces the MANETs, biometrics, and IDS. Because of no-fixed infrastructure available and the dynamic nature of ad hoc networks, user authentication and verification are core requirements for integrity, confidentiality and non-repudiation. Biometrics is the only factor that can directly check the identity of the user, which makes it an potential way to be used in user authentication in MANETs. There are two classes of user authentication in MANETs: user-to-device and user-to-network. As the second line of defense, intrusion detection system continuously or periodically monitors the network for unusual activity and triggers the appropriate response. A complete security scheme should combine the authentication, IDS and relevant responses.

A significant amount of work has been done on biometric-based continuous authentication such as using Dynamic Bayesian Network model [17] and using HMM model [3]. Although some researchers examined the security requirements of MANETs and proposed some promising decentralized user authentication schemes [1][2][5], there is still a lot of work to do for user authentication in MANETs. 


\section{Chapter 3}

\section{MDP and POMDP}

In our daily life, many problems, such as optimal control problem and speech recognition, can be formulated into Markov decision process (MDP) or partially observable decision process (POMDP). The authentication schemes we will propose in Chapter 4 are also based on POMDP mathematics models. Therefore, we will give a brief introduction of MDP and POMDP in this chapter.

\subsection{Markov Decision Process}

\subsubsection{MDP Model}

Decision making is an important part in everyday life such as changing the components or not in machine-maintenance problem [19]. Making the correct decision can help to utilize the system resources, acquire the maximum revenue, etc. Normally decision making is based on the system environment, the interrelationship between the system components, the previous experience. However, with the system becomes more complex and the degree of uncertainty increases, it is more difficult to make an appropriate decision by human. Hence, the automated decision making is desirable in 
many sophisticated applications in which the human decision making is not feasible or impossible to formalize. Each decision is associated with a revenue here we call it reward. Best decision is not necessarily the action that brings the best immediate reward. The immediate reward is easier to check than long term reward. But sometimes the poor immediate reward can bring better long term reward. So people are interested in making decision to acquire the right tradeoffs between immediate rewards and long term gains. Markov decision process provides a way to automatically process the decision problems.

MDP is a simple quadruple $<S, A, P, R>$ model, which consists of a finite set of states, a finite set of actions, the effects of each action-immediate reward pair.

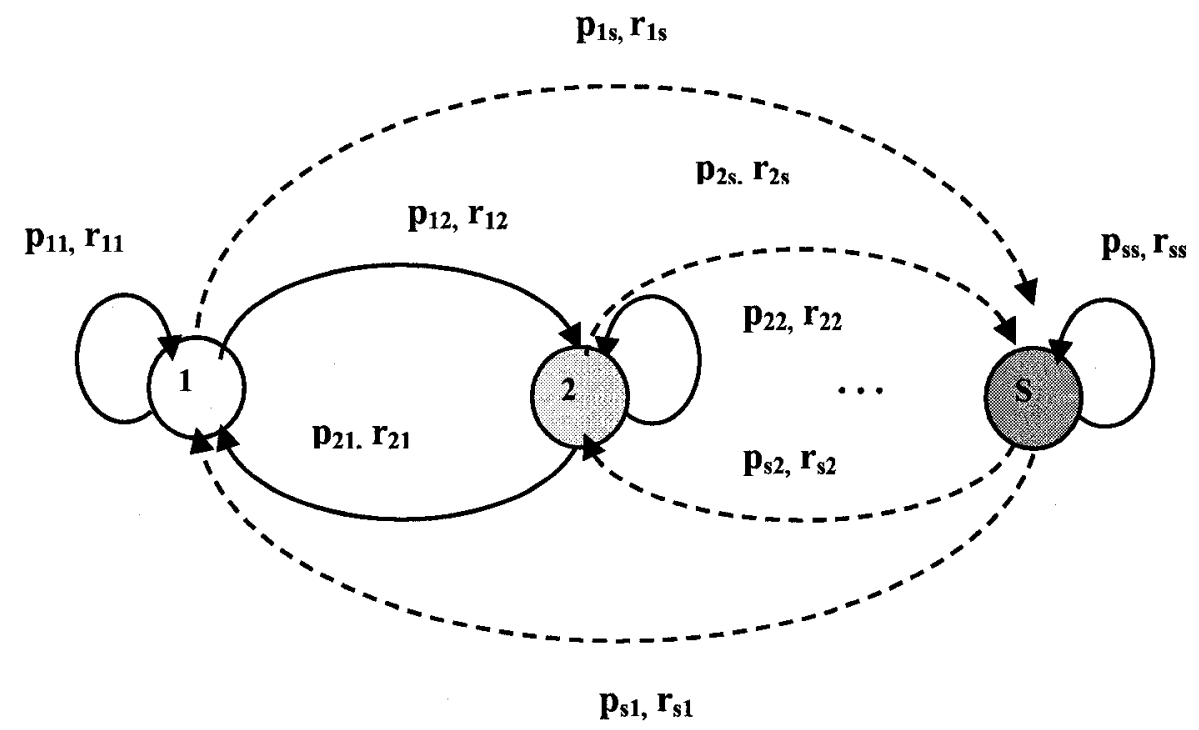

Fig. 3.1 Markov decision process.

- States $S$ : The states denotes the way the system currently stay in. Though in most cases, the system stays in a continuous states, it is convenient to discretize these continuous spaces. $S$ stands for the system state space, and $s_{i}$ denotes the state of $i$. 
- Actions $A$ : The actions are a set of decisions that can be taken at each time point. $A$ denotes the action space with action $a_{i}$.

- Transitions P: Different actions have different effects on the system states. The transition defines how each action changes the system states. Probability $p_{i j}^{a}=\operatorname{Pr}\left(S^{k+1}=j \mid S^{k}=i, A^{k}=a\right)$ is used in MDP to specify the transition probability from the current system state $i$ to another system state $j$, here $k$ denotes the time instant.

- Immediate rewards $R$ : Since different actions changes the system state in different way, we use immediate reward to measure an action's value which will be used to compare the effects of actions. $r_{i j}^{a}$ represents the immediate reward when action a is performed while system stays in state $s_{i}$ and moves to state $s_{j}$.

Figure 3.1 is a valid representation of MDP. One important characteristic of MDP model is that the next state of the system is solely determined by the current state and the current action. In practise, there are some forms of uncertainty for MDP: the results of actions may not always have the same effects; our perceptions of the system being controlled are not always very accurate. Therefore, we let $q_{i}^{a}$ represent the expected rewards, what we would expect to receive on average in the long term:

$$
q_{i}^{a}=\sum_{j} p_{i j}^{a} r_{i j}^{a}
$$

where $r_{i j}^{a}$ are the actual rewards that would be received. The value of $q_{i}^{a}$ only depends on the current system state and action chosen.

Based on the above definition, a system will evolve as follows: at each time instant, the system is in a particular state $s_{i}$, an action $a$ is taken and the system will move to another system state $s_{j}$ with the probability $p_{i j}$, an immediate reward $r_{i j}^{a}$ is associated 
with this specific process.

\subsubsection{Policies}

A policy is the solution to a MDP and it specifies the best actions to take for each of system states. A policy completely specifies the actions which are appropriate for all system states that possibly occur during the system process.

- Deterministic or stochastic: A policy could be deterministic or stochastic. A deterministic policy specifies only one action to execute for a system state. A stochastic policy specifies a number of actions to execute for a system state, and each action is associated with a probability distribution over the set of actions. A deterministic policy is a special case of stochastic policy since its action to execute has the probability 1 for a specific system state.

- Stationary or non-stationary: A policy can be classified into stationary and non-stationary. A stationary policy is irrelevant with time and same policy will be applied to the system when it is used. We use $\delta=\{\delta, \delta, \ldots, \delta\}$ to denote the stationary policy. In other words, the policy only depends on the system state. A non-stationary policy depends on both time and system state. $\delta=\left\{\delta_{1}, \delta_{2}, \ldots, \delta_{k}\right\}_{s}$ used to denote the non-stationary policy, here $k$ is the time instant.

- Finite or infinite: The time axis is divided into slots of equal duration which correspond to the time interval between two continuous decisions, and this time interval is also known as horizon in this paper. The finite-horizon problem is the one in which decisions need to be made only for finite time steps. The policy for finite horizons consists of a sequence of deterministic policies, one for each time instant, denoted as $\delta=\left\{\delta_{1}, \delta_{2}, \ldots, \delta_{k}\right\}$. Another problem is infinite- 
horizon problem in which we do not know the number of time horizons over which the decision need to be made. Under this situation, a stationary policy $\delta=\{\delta, \delta, \ldots, \delta\}$ is needed. At any time instant, the policy $\delta$ is same because there are unbounded amount of time left for both time instant $k$ and $k+1$.

\subsubsection{Optimality Criteria}

The immediate reward function $q_{i}^{a}$ helps to guide the decision, but maximum immediate reward does not mean the maximum long term reward. There are many ways to balance the trade-off between the immediate rewards and long term accumulated rewards. Expected future discounted reward [20] is considered in this paper:

$$
E\left[\sum_{k=0}^{K-1} \beta^{k} q_{S^{k}}^{A^{k}}\right], 0 \leq \beta \leq 1,
$$

where $S^{k}$ and $A^{k}$ are the random variables for the state and action chosen at time instant $k \beta$ is a discount factor that means rewards receives later in time will have less value than an equivalent reward received closer to the present.

For the infinite horizon problem, equation (3.2) can be re-written as:

$$
E\left[\sum_{k=0}^{\infty} \beta^{k} q_{S^{k}}^{A^{k}}\right], 0 \leq \beta \leq 1,
$$

here $0 \leq \beta \leq 1$ is used to ensure that the expectation is bounded with the same meaning as in equation (3.2).

With the definitions above, the aim in solving the MDP is to find a control policy which maximizes expected future discounted reward, and this policy is called optimal policy. 


\subsubsection{Value Functions}

The optimal policy for MDP can acquire a maximum expected future discounted reward. We use value function to compute a mapping from states to actions, which represents the best actions to take for each state.

\section{Finite Horizon}

For a finite MDP with non-stationary process, we can calculate the value function with the recursive equation. We define $V_{k}^{\delta^{k}}(s)$ as the value of starting in state $s$ and executing the policy $\delta^{k}$ for $K-k$ time steps. The reward is the immediate reward received for the current state, current action and next state $r_{i j}^{a}$, plus the value of state $s_{j}$ with one less step remaining $V_{k+1}^{\delta k+1} s(j)$ :

$$
V_{k}^{\delta^{k}}\left(s_{i}\right)=q_{i}^{\delta^{k}\left(s_{i}\right)}+\beta \sum_{j} p_{i j}^{\delta^{k}\left(s_{i}\right)} V_{k+1}^{\delta^{k+1}}\left(s_{j}\right), q_{i}^{\delta^{k}\left(s_{i}\right)} \text { is defined in Equation(3.1). }
$$

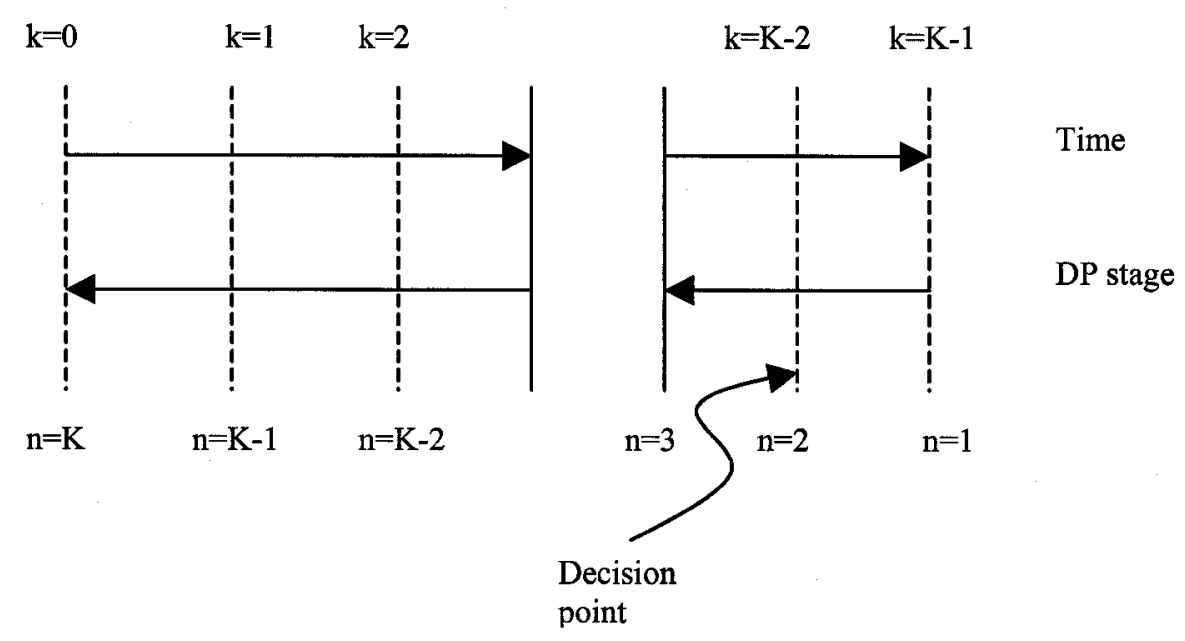

Fig. 3.2 Relationship between time and dynamic programming indices

In order to calculate the equation effectively, we will use dynamic programming to compute the optimal policy. In other words, compute the equation (3.4) backward from time $K$ down to time 0 . The relationship between time and dynamic program- 
ming indices is shown in Figure 3.2 [20]. The value function can be rewritten as:

$$
V_{n}^{\delta_{n}}\left(s_{i}\right)=q_{i}^{\delta_{n}\left(s_{i}\right)}+\beta \sum_{j} p_{i j}^{\delta_{n}\left(s_{i}\right)} V_{n-1}^{\delta_{n-1}}\left(s_{j}\right)
$$

\section{Infinite Horizon}

For the problem with stationary policy over infinite horizon, the policy $\delta$ is always same at any time instant. The value function can be written as:

$$
V^{\delta}\left(s_{i}\right)=q_{i}^{\delta\left(s_{i}\right)}+\beta \sum_{j} p_{i j}^{\delta\left(s_{i}\right)} V^{\delta}\left(s_{j}\right)
$$

These value functions help us to compute a metric for the policies and compare the policies to get an optimal policy, the optimal mapping from a state to an action.

\subsection{Partially Observable Markov Decision Process}

\subsubsection{POMDP Model}

In MDP, the states of the system are completely known at all times and the decisions are made based on those states. However, at most times, the system states are not directly observed and only some observations that give a hint of the current system state. This general model is called POMDP, and MDP we discussed previously are called completely observable Markov decision process (CO-MDP).

POMDP is really just a MDP which is defined by a hex-tuple $<S, A, P, \Theta, B, R>$. The definitions of $S, A, P$ are the same as those in MDP. $S$ stands for a finite set of states with state $i$ denoted by $s_{i}$. A stands for a finite set of actions with action $i$ denoted by $a_{i} . P$ stands for transition probabilities for each action in each state and $p_{i j}^{a}$ denotes the probability that system moves from state $s_{i}$ to state $s_{j}$ when action $a$ was performed. $\Theta, B$ are two definitions that are added to POMDP. $\Theta$ stands for 
a finite set of observations where $\theta_{i}$ denotes the observation $i . B$ is the observation model in which $b_{j \theta}^{a}$ denotes the probability that $\theta$ was observed when the system state is $s_{j}$ and last action taken at time $k-1$ was $a$. Individual observation probability $b_{j \theta}^{a}$ is defined as $b_{j \theta}^{a}=\operatorname{Pr}\left(\Theta^{k}=\theta \mid S^{k}=j, A^{k-1}=a\right)$, and the observation is dependent on the resulting state in the state transition. In POMDP, $R$ is redefined and it stands for the immediate reward. $r_{i j \theta}^{a}$ denotes the immediate reward received at time $k$ for performing action $a$ at time $k-1$, and the system state moves from $s_{i}$ at time $k-1$ to state $s_{j}$ at time $k$, and the observation is $\theta$ at time $k$. Like the definition in MDP, we still use $q_{i}^{a}$ to denote the expected immediate reward:

$$
q_{i}^{a}=\sum_{j, \theta} p_{i j}^{a} b_{j \theta}^{a} r_{i j \theta}^{a}
$$

The POMDP model is shown in Figure 3.3. In each system state, the observation is made after an action is taken. The system states cannot be observed directly at each time instant, and only observations which give hints of the system states can be acquired, $k$ stands for time instant at which decision need to be made.

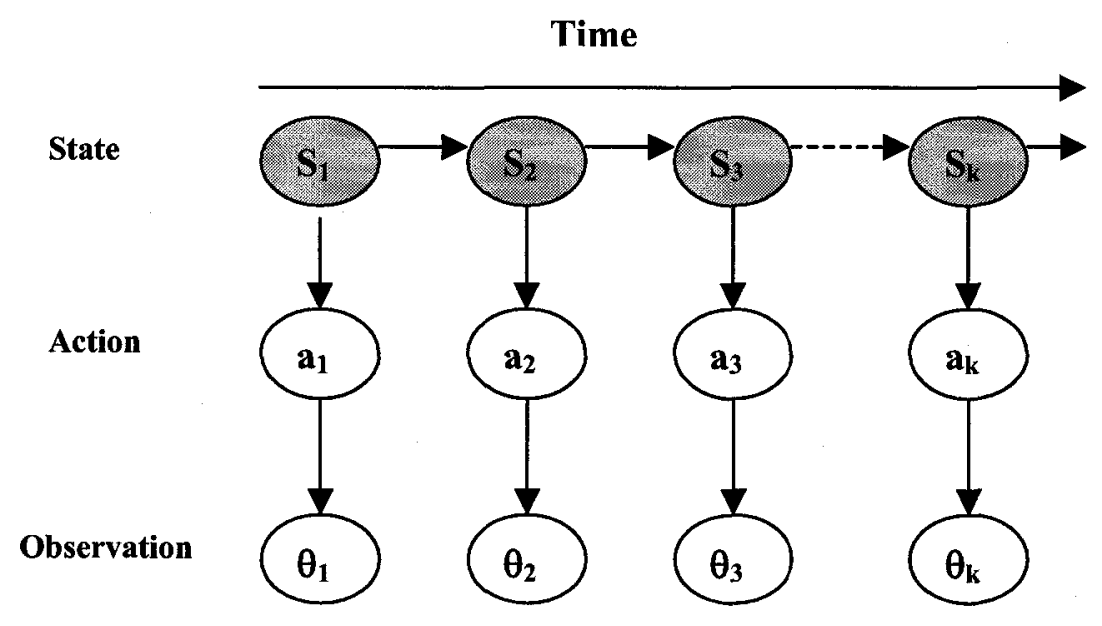

Fig. 3.3 POMDP model. 

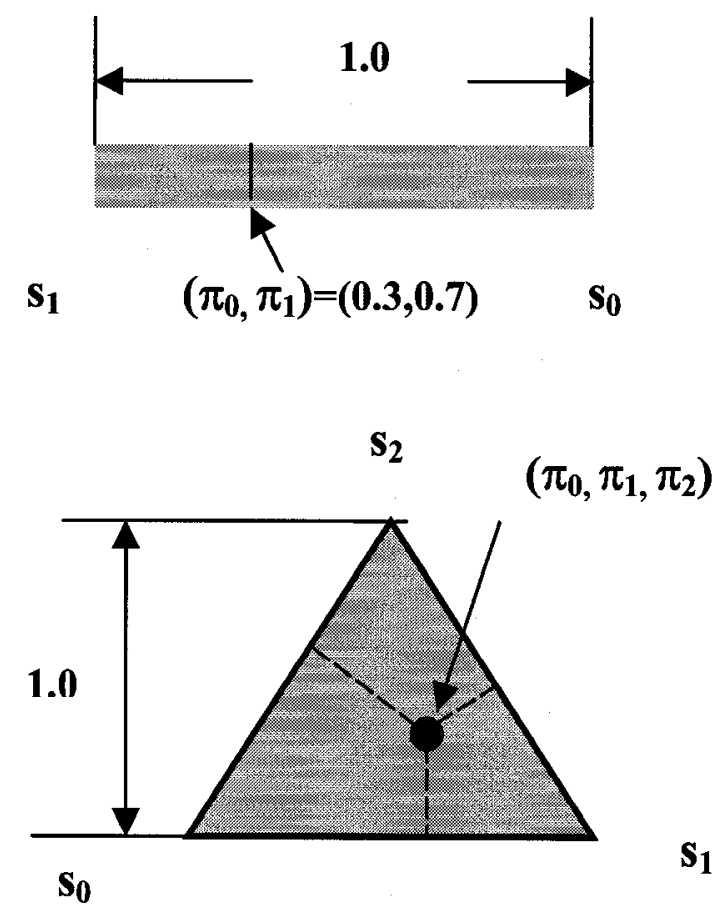

Fig. 3.4 Information space with 2 states and 3 states. 


\subsubsection{Information State}

In MDP, the optimal policy is defined based on the history of actions and system states. The properties of MDP provide the solution to the problem of making decision by finding a simple mapping from states to actions. But in POMDP, the states cannot be observed directly and making decision only based on the observations is arbitrarily poor. It is fortunate that we can derive a summary statistic for the entire history of a process with the definition of information state, which is used for decision making.

We will refer to a probability distribution over states as an information state and the entire probability space (the set of all possible probability distributions) as the information space. The information spaces with 2 and 3 states are shown in Figure 3.4. The corners are the Markov chain states $s_{0}, s_{1}$, and $s_{2}$. For a system with 2 states, its information space is a one-dimension line, the thickness of the line is only used for clarifying the explanations. The distance from the left axis is the first component $s_{0}$ and the distance from the right is the second component $s_{1}$; for the system with 3 states, its information space is a two-dimension triangle, the value of a point in the information space can be obtained by the perpendicular distance to the sides of the triangle. An information state is a sufficient statistic for the history, which means that decision making can be based on the information state, denoted by a vector $\pi^{k}=\left(\pi_{1}^{k}, \pi_{2}^{k}, \ldots, \pi_{|s|}^{k}\right)^{\prime}, 1_{S}^{\prime} \pi^{k}=1$, where $k$ stands for the time instant, $|S|$ denotes the number of states, $\pi_{i}$ denotes the probability that system is currently in state $s_{i}, 1_{S}$ represents an $S$-dimensional vector of ones.

One important property of the information state is that it can be easily updated with Bayes rule by incorporating one additional observation into the history.

$$
\pi_{j}=\frac{\sum_{i} \pi_{i}^{\prime} p_{i j}^{a} b_{j \theta}^{a}}{\sum_{i, j} \pi_{i}^{\prime} p_{i j}^{a} b_{j \theta}^{a}}
$$


The new belief state will be a vector of probabilities computed according to the above formula. The information states capture all the history information which is represented as $\left\{a^{0}, a^{1}, \ldots, a^{k-1}, \theta^{1}, \theta^{2}, \ldots, \theta^{k}\right\}$ at time $k$. Therefore, we can save all the past actions and observations by constantly updating the information state $\pi$, and it is reasonable to make decisions according to the information state. In fact, we can convert a continuous POMDP problem into a discrete space CO-MDP problem. A model of dynamic POMDP is shown in Figure 3.5. The action is generated as a function of information state and the system state is observed after the action is taken. The information state will be updated with the latest observation.

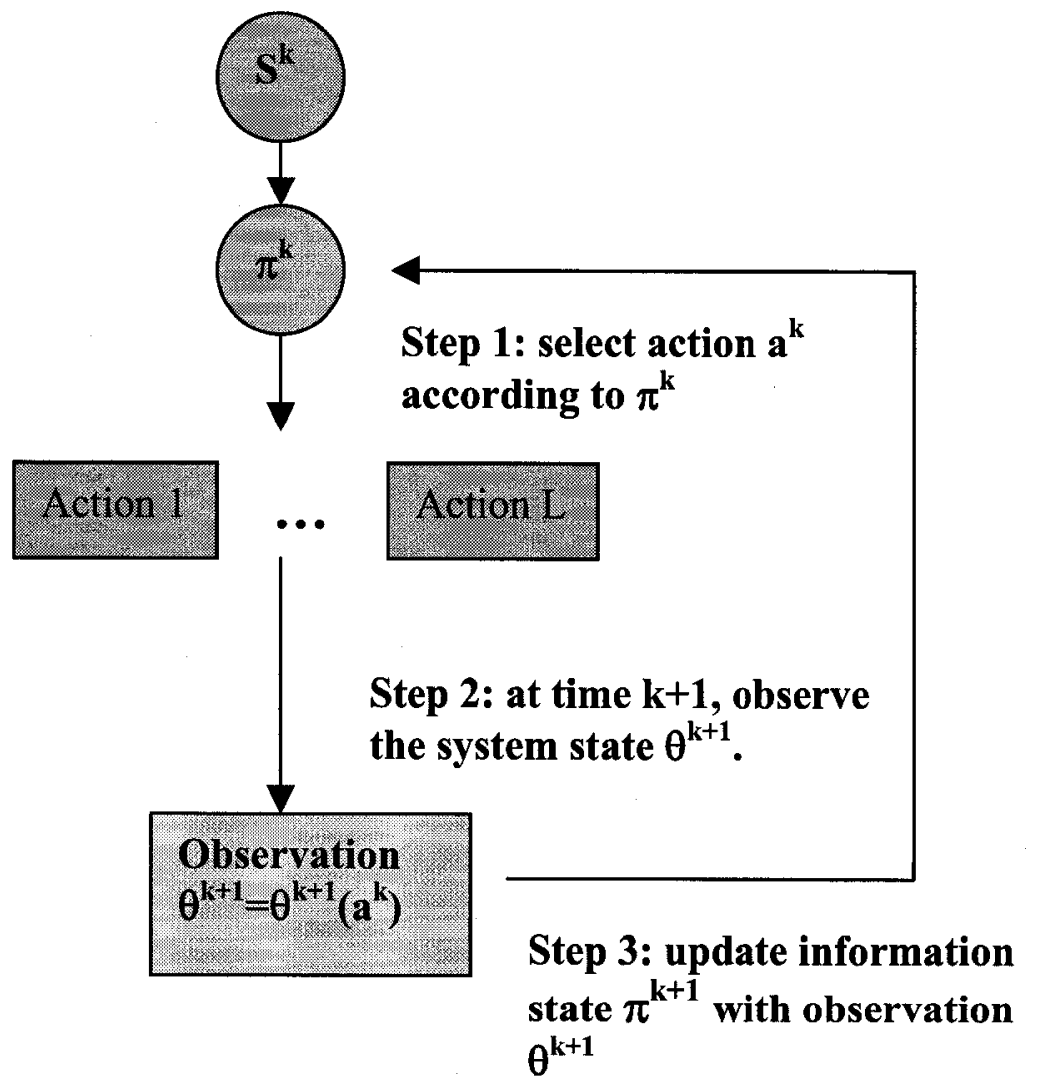

Fig. 3.5 POMDP dynamic model. 


\subsubsection{Policies and Value Function of POMDP}

\section{POMDP Policy}

In CO-MDP model, a policy is a mapping from states to actions. Since state space and actions are finite, the value function and policy are easy to calculate. But in POMDP, the underlying states cannot be observed directly, the continuous information statethe likelihood of being in each state is used instead to make decision. Our task is to compute a policy that obtains, based on the information state, the maximum expected reward for a single action. The POMDP policy can be derived from a value function which is defined over the entire information space.

\section{Value Functions}

For the $k$-horizon value function of POMDP, we need to consider more extra complications than MDP: (i) We need to consider the weighted average value over the information states. (ii) We need to consider the weighted average value over all possible observations. (iii) We need to consider the value that is based on the information state for the next step, and the information state for the next step is transformed from the current information state, action and observation. The value function can be defined as follows:

$$
V_{n}^{*}(\pi)=\max _{a \in A}\left[\sum_{i} \pi_{i} \sum_{j} p_{i j}^{a} \sum_{\theta} b_{j \theta}^{a}\left(r_{i j \theta}^{a}+\beta V_{n-1}^{*}\left(\pi_{\theta}^{a}\right)\right)\right], 0 \leq \beta \leq 1
$$

This equation can be simplified with substitution of (3.7) for the $q_{i}^{a}$ values:

$$
V_{n}^{*}(\pi)=\max _{a \in A} \sum_{i} \pi_{i} q_{i}^{a}+\beta \sum_{i, j, \theta} \pi_{i} p_{i j}^{a} b_{j \theta}^{a} V_{n-1}^{*}\left(\pi_{\theta}^{a}\right), 0 \leq \beta \leq 1
$$


Compared to the value function in MDP, the value function in POMDP is slightly complicated: $V_{n}^{*}(\pi)$ is the expected reward when observation $\theta$ is acquired after action $a$ is taken in state $\pi$ and when there are $n$ decisions remained.

\section{Properties of Finite POMDP}

Solving the value function of POMDP would get messy because the continuous information space is used. Fortunately, Sondik and Anthony showed that the optimal finite horizon value function is piecewise linear and convex $(P W L C)$ for any horizon $K$ [19] [20]. This means for each horizon $k$, the value function can be represented with a set of linear segments $n_{k}=\left\{n_{k}^{1}, n_{k}^{2}, \ldots, n_{k}^{H}\right\}$ :

$$
V_{k}^{*}(\pi)=\max _{h \in H} \sum_{i} \pi_{i} n_{i, k}^{h}
$$

here $i \in\{1,2, \ldots, S\}$ for $S$-state POMDP problem. For POMDP problem with two states, the relationship between PWLC value function and value function over information space is shown in Figure 3.6 and Figure 3.7. The value function shown in Figure 3.6 can be represented with the upper surface of 3 vectors $n^{1}, n^{2}$ and $n^{3}$ in Figure 3.7. For 3 or more states POMDP model, their value functions can be represented with hyper-plane through information space and each hyper-plane can be represented with a vector of numbers, which are the coefficients of the hyper-plane. The value of information point is simply the dot product of the two vectors shown as follows:

$$
\sum_{i} n_{i} \pi_{i}=n_{0} \pi_{0}+n_{1} \pi_{1}+\ldots+n_{S} \pi_{S}
$$


At each time instant $k$, the value function of the information space can be represented with a set of vectors, and to find the value of an information state, we only need to find the vector that has the largest dot product with the information state.

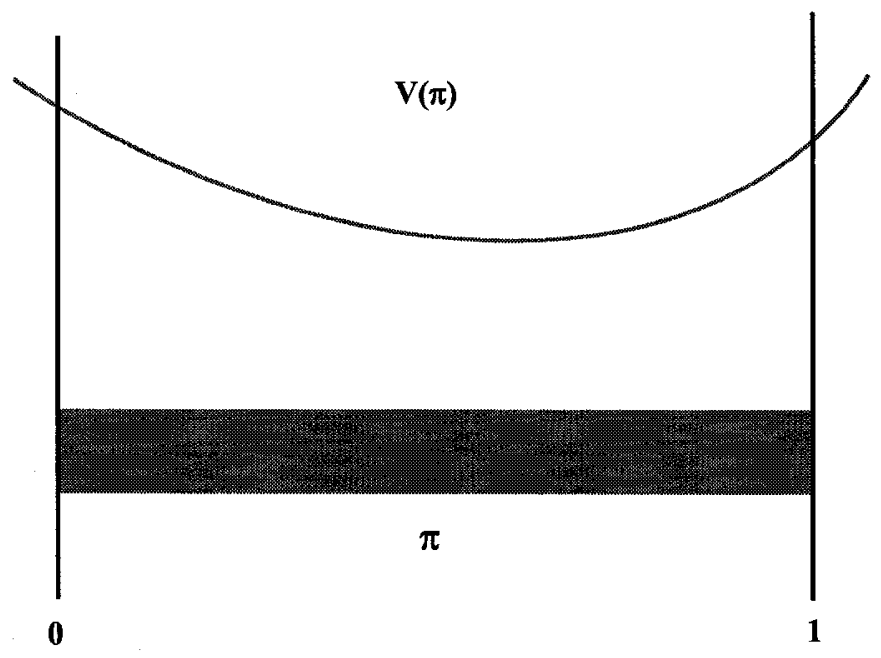

Fig. 3.6 Value function over information space.

From Figure 3.7, we can see that these 3 vectors partition the information space into 3 parts: For the left part, $n^{1}$ will acquire the largest value; for the middle part, $n^{2}$ will acquire the largest value and $n^{3}$ will get the largest value on the right part.

Another important thing is that each of the $n$-vectors for time step $k$ has an associated action that gave rise to it. When we construct value function by maximizing the value of dot products between vectors and information states, we also need to keep the track of the specific action $a$ which gave rise to it for each $n$ vector. This combination of vector-action pairs gives us a way to find the optimal policy for POMDP problem: Perform the maximization over all $n_{k}$, and take the associate action of that vector. 


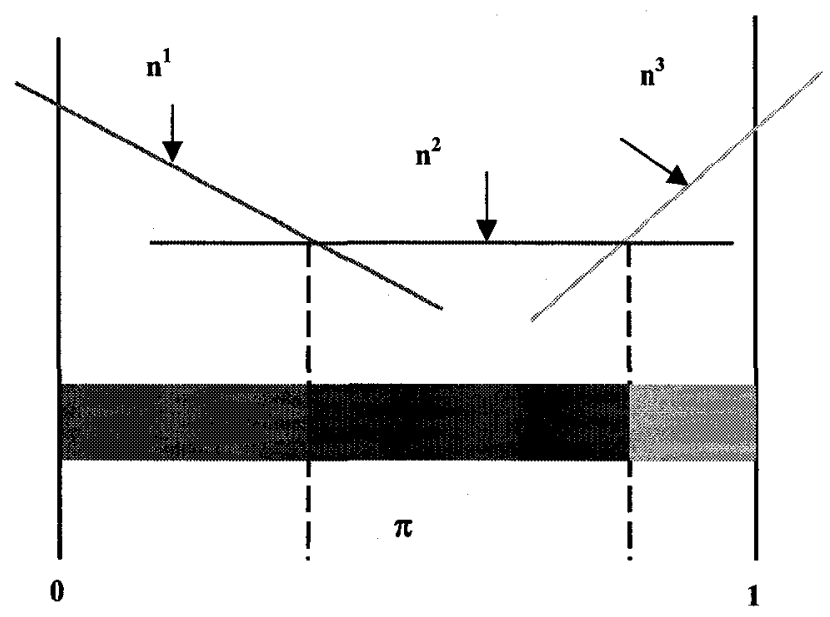

Fig. 3.7 PWLC value function.

\section{Infinite POMDP}

For infinite horizon POMDP, the value function can be expressed as:

$$
V^{*}(\pi)=\max _{a \in A} \sum_{i} \pi_{i} q_{i}^{a}+\beta \sum_{i, j, \theta} \pi_{i} p_{i j}^{a} b_{j \theta}^{a} V^{*}\left(\pi_{\theta}^{a}\right), 0 \leq \beta \leq 1
$$

Even though the optimal value function of finite POMDP is PWLC, and

$$
\lim _{n \rightarrow \infty}\left\|V_{n}^{*}(\cdot)-V^{*}(\cdot)\right\|=0
$$

it does not mean that all the value functions of infinite POMDP are piecewise linear [21]. Theoretically, for the piecewise and linear POMDP value function, $V^{*}(\cdot)$ can be approximated with large enough horizon $V_{n}(\cdot)$. In practise, non-linear value function can also be approximated with a piecewise linear function as closely as possible.

Despite the uncertainty about the value function of infinite POMDP, the $V^{*}(\cdot)$ is always convex [20]. 


\section{Algorithms Overview}

There are several linear programming based algorithms in the POMDP literature such as:

- Monahan's algorithm [20]: This algorithm is both easy to understand and easy to implement. However, it can only used to solve the problems with a few states.

- Sondik's algorithm [19] [21]: Sondik presented the first solution techniques to find optimal policies for general POMDP problems, and all the other algorithms described later are derived from it.

- Cheng's algorithms [22]: In Cheng's algorithms, the use of linear programming is eliminated and interior point method is used instead to find the corner points of the regions defined on the information space.

- Witness algorithm [20]: This algorithm is derived directly from Cheng's algorithm. There are two main differences from Cheng's algorithm: First, it doesn't deal directly with an approximation to the value function. Instead, it defines related value functions and then builds these functions up from approximations. Second, It uses the different ways that points are found to refine the approximation.

- Incremental pruning algorithm [23]: Like Witness, this algorithm breaks down the problem into constructing the $\Gamma_{n}^{a}$ sets individually and it constructs each vector in an incremental fashion which reduces the computational complexity significantly.

All algorithms have a few things in common: (i) They all are performed as a dynamic program. (ii) All of the algorithms try to find the set of vectors that define both the 
value functions and the optimal policy at each time step. (iii) Each of the vectors in $V_{t}^{*}$ will have a single control action $a$ associated with it. The only difference among these algorithms is the ways to compute a single dynamic programming step. The detail information and correspondent programming codes of these algorithms can be found in [20] and [24]. 


\subsection{Summary}

In this chapter we introduced Markov decision model and partially observable Markov decision model. We first formulated MDP as a simple quadruple $\langle S, A, P, R\rangle$, and the value function used to solve the MDP problem to get the optimal policy is introduced. Then we defined the POMDP as a hex-tuple $<S, A, P, \Theta, B, R>$ model. By adopting the definition of information space, the discrete POMDP problem can be converted into a continuous MDP problem. Therefore, all the theories and results used for MDP can be used for POMDP problems. The value function of POMDP is piecewise linear and convex (PWLC), and it is very useful because it allows the value function to be represented using finite resources. Finally, the common algorithms used to solve POMDP problems were introduced in this chapter. 


\section{Chapter 4}

\section{Proposed Authentication Schemes}

\subsection{Continuous User-to-device Authentication}

\subsubsection{Necessity of User-to-device Authentication in MANETs}

Most traditional authentication systems verify a user during initial login. However, for tactical MANETs in hostile environment where chances of node capture are high, it is important to verify the presence of the authentic user continuously during the lifetime of MANETs. The frequency of applying re-authentication depends on the severity of the environment, system security requirements and resource constraints $[3][17]$.

The experience in security of wireline networks indicates the importance of reauthentication because there are always some weak points in the system, no matter what is used for authentication. This is especially true for MANETs, given the low physical security of mobile devices.

After a re-authentication process, only authentic users can continue using the network resources and compromised users will be excluded [15].

In this Chapter, we formulate the whole state of the device as a partially observ- 


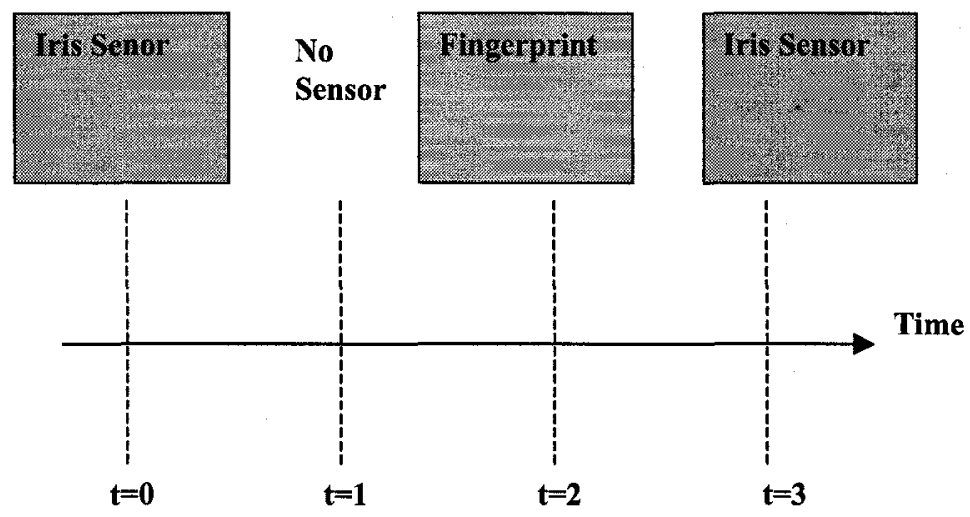

Fig. 4.1 Sensor scheduling.

able Markov decision process (POMDP) [20]. Each device is equipped with several biosensors which are used for re-authentication. Our scheme can automatically decide which biosensor will be used at each time instant. The optimal policy (the sensor scheduling is shown in Figure 4.1) can be acquired by solving POMDP with dynamic programming-based hidden Markov model scheduling algorithms.

\subsubsection{System Model}

In tactical MANETs, user and device are generally tightly coupled. If the user-todevice authentication fails, it means that the device is not in right hand and appropriate measures can be taken accordingly. Moreover, in a tactical operation, users intervention should be kept minimal [25]. The proposed model below applies multimodal biometrics to user-to-device authentication to address the above problems in tactical MANETs. In this model, the time axis is divided into slots of equal duration which corresponds to the time interval between two continuous authentications. Our biosensor selecting problem is assumed to evolve as a $S$-state POMDP. Under this model, the state of the device evolves according to a discrete-time, $S$-state first order 
Markov chain $\left\{X^{k}\right\}$, where $k$ denotes the authentication time instant. The state of the device at the time instant is $X^{k}$ with state space $\left\{e_{1}, \ldots, e_{S}\right\}$. Here, $e_{i}$ denotes the $S$-dimensional unit vector with 1 in the ith position and zeros elsewhere. The $S \times S$ transition probability matrix $P$ is defined as:

$$
\begin{aligned}
& P=\left[p_{i j}\right]_{S \times S}, \text { where } p_{i j}=\operatorname{Pr}\left(X^{k}=e_{j} \mid X^{k-1}=e_{i}\right) \\
& i, j \in\{1, \ldots, S\} .
\end{aligned}
$$

Assume we have $L$ biosensors to be used for continuous authentication and one biosensor or no biosensor will be chosen at each time instant. We can safely extend this scheme to several biosensors used at the same time. Let $a^{k} \in\{1, \ldots, L\}$ denotes the biosensor selected at time $k$, and $y^{k}\left(a^{k}\right)$ denotes the observation of this biosensor. The observations of the $l$ th biosensor belong to a finite set of symbols $\left\{O_{1}(l), O_{2}(l), \ldots, O_{M_{l}}(l)\right\}$ and $\left|M_{l}\right|$ denotes the number of possible observations of the $l t h$ biosensor. When system state is $e_{i}$, the $l t h$ biosensor is picked at time $k$, the probability of observation $m$ will be got from the $l t h$ biosensor is denoted as:

$$
\begin{aligned}
b_{i}\left(a^{k}\right. & \left.=l, y^{k}=O_{m}(l)\right)=\operatorname{Pr}\left(y^{k}\left(a^{k}\right)=O_{m}\left(a^{k}\right) \mid X^{k}\right. \\
& \left.=e_{i}, a^{k}=l\right), i=1,2, \ldots, S
\end{aligned}
$$

Define the observation matrix as: $B\left(a^{k}, O_{m}\left(a^{k}\right)\right)=$

$$
\left(\begin{array}{ccccc}
b_{1}\left(a^{k}, O_{m}\left(a^{k}\right)\right) & 0 & 0 & \cdots & 0 \\
0 & b_{2}\left(a^{k}, O_{m}\left(a^{k}\right)\right) & 0 & \cdots & \vdots \\
0 & 0 & \ddots & 0 & \vdots \\
\vdots & \vdots & 0 & \ddots & 0 \\
0 & \cdots & \cdots & 0 & b_{S}\left(a^{k}, O_{m}\left(a^{k}\right)\right)
\end{array}\right)
$$


It denotes the probabilities of the observation $m$ acquired when the biosensor $a^{k}$ is picked at time $k$ given each state of Markov chain. The possible observations from the biosensors could be acceptance if the result of authentication is accepted, rejection if the result of authentication is rejected, and nothing if no biosensor is applied. Note that the state of the device is not directly observed, thus the state of the device is a hidden Markov model.

There are costs associated with biosensor usage: the energy consumed for computation, the information stolen if a wrong authentication result is acquired, and etc.

\subsubsection{Information State}

According to the definition of information state in Chapter 3, we let $F$ denote the set of all information states $\pi$ and define $\pi^{k},(k$ denotes time instant) as:

$$
\begin{aligned}
\pi_{i}^{k} & =\operatorname{Pr}\left(X^{k}=e_{i} \mid Y^{k}\right), i=1,2, \ldots, S \\
F & =\left\{1_{S}^{\prime} \pi=1,0 \leq \pi_{i} \leq 1 .\right\}
\end{aligned}
$$

Where $Y^{k}=\left\{a^{1}, a^{2}, \ldots, a^{k}, y^{1}, y^{2}, \ldots, y^{k}\right\}$ represents the information available at time $k$. $1_{S}$ represents the $S$-dimensional vector of ones. The important thing of information state is that it can be easily updated after each state transition to incorporate one additional step information into history [26]:

$$
\pi^{k+1}=\frac{B\left(a^{k+1}, y^{k+1}\left(a^{k+1}\right)\right) P^{\prime} \pi^{k}}{1_{S}^{\prime} B\left(a^{k+1}, y^{k+1}\left(a^{k+1}\right)\right) P^{\prime} \pi^{k}}
$$


The initial probability vector of Markov chain is denoted as:

$$
\pi^{0}=\left[\pi_{i}^{0}\right]_{S \times 1}, \text { where } \pi_{i}^{0}=\operatorname{Pr}\left(X^{0}=i\right), i \in\{1, \ldots, S\}
$$

By using the connection between information state and device state, a biosensor can be picked based on the information state at each time instant rather than exact device state.

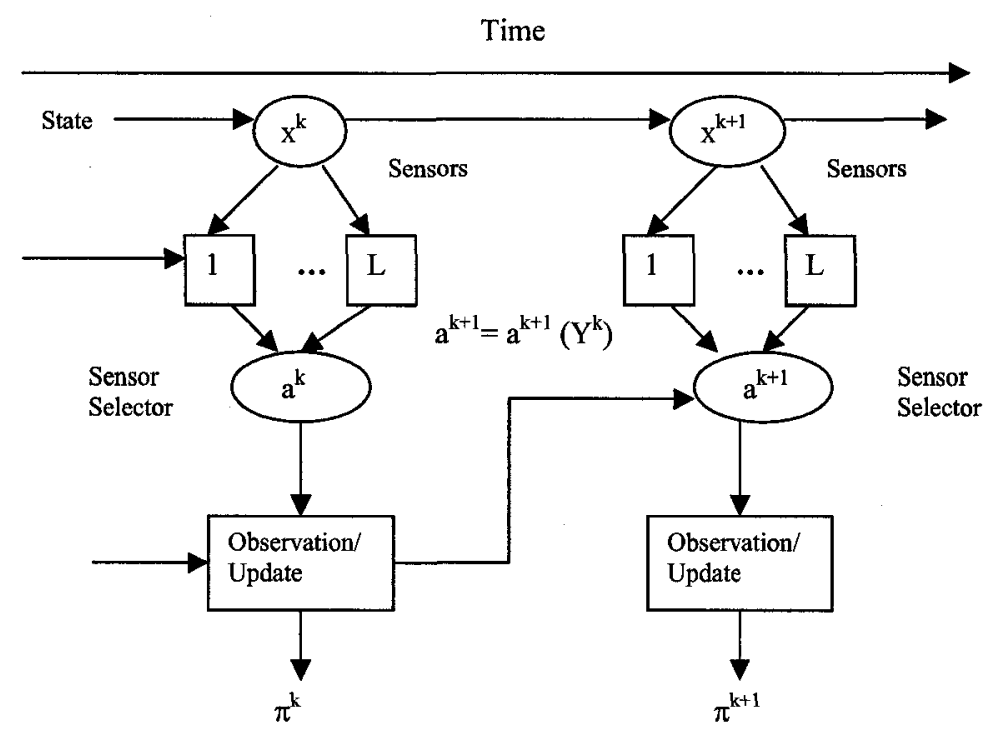

Fig. 4.2 HMM sensor scheduling and information state update.

\subsubsection{Sensor Scheduling Architecture}

With all information above, the sensor scheduling procedure can be briefly summarized as three steps shown in Figure 4.2[26]:

- Scheduling: Based on the history information $Y^{k}$, find the optimal biosensor $a^{k+1}$ that will be used at the next horizon. 
- Observation: Observe the output of the optimal biosensor $y^{k+1}\left(a^{k+1}\right)$ at next horizon.

- Update: Update the information state $\pi^{k+1}$ by using the latest observation $y^{k+1}$, this new information state will be used to judge the result of authentication.

With the above scheme, we can find the sensor scheduling sequence

$$
a=\left\{a^{1}, a^{2}, \cdots, a^{N}\right\}, N \text { denotes the time instant. }
$$

\subsubsection{Cost Definition}

In the previous introduction of POMDP, we use reward to build the value function. In this thesis, we consider the cost and use it to build our scheme value function. Since cost is the negative reward, we only need to minimize this value function to compute the optimal policy.

At time $k$, based on the history information $Y^{k}\left(a^{k}\right)$, the biosensor $a^{k+1}=l$ is selected. Then the instantaneous cost incurred at time $k$ is:

$$
\underbrace{u^{k}(l)\left\|X^{k}-\pi^{k}\right\|_{D}}_{\text {Part1 }}+\underbrace{c^{k}\left(X^{k}, l\right)}_{\text {Part2 }}
$$

Here $u^{k}(l), l=1,2, \ldots, L$ are positive scalar weights, the $D$ is a quantized norm. In this paper, we select $D=l_{2}$. Then Part 1 means the square error (Euclidean distance) in the state estimation at time $k$ during to choosing biosensors $a^{1}, a^{2}, \ldots, a^{k}$. In biometric-based authentication, the bigger the state estimation error is, the FRR or the FAR will be larger. Part 2 denotes the instantaneous cost of using the sensor $a^{k+1}=l$ when the device state is $X^{k}$. In MANETs, we consider this cost as energy consumption, information leaking, and etc. There are many ways to make the tradeoff 
between immediate cost and long term cost. Here we only consider the expected future discounted cost. The cumulated cost [26] from time 1 to $N$ can be expressed as:

$$
J_{a}=E\left\{\sum_{k=0}^{N-1} u^{k}\left(a^{k+1}\right)\left\|X^{k}-\pi^{k}\right\|_{D}+\sum_{k=0}^{N-1} c^{k}\left(X^{k}, a^{k+1}\right)+u^{N}\left\|x^{N}-\pi^{N}\right\|_{D}\right\}
$$

And for infinite horizon discounted cost, the cost can be expressed as:

$$
J_{a}=E\left\{\sum_{k=0}^{\infty} \beta^{k}\left[u\left(a^{k+1}\right)\left\|X^{k}-\pi^{k}\right\|_{D}+c\left(X^{k}, a^{k+1}\right)\right]\right\}
$$

Where the constraint $0 \leq \beta<1$ ensures that the expectation is bounded. What we need to do is to minimize this cost by finding the optimal sensor schedule (optimal policy).

Considering the information state incorporated into POMDP, and for convenience, we define the cost into a $S$-dimensional vector

$$
c^{k}\left(a^{k+1}\right)=\left[c^{k}\left(e_{1}, a^{k+1}\right), \ldots, c^{k}\left(e_{S}, a^{k+1}\right)\right]^{\prime}
$$

the cumulated cost above can be rewritten as:

$$
J_{a}=E\left\{\sum_{k=0}^{N-1} C^{k}\left(\pi^{k}, a^{k+1}\right)+C^{N}\left(\pi^{N}\right)\right\}
$$

where $a^{k+1}=a^{k+1}\left(\pi^{k}\right)$

$$
\begin{aligned}
& C^{N}\left(\pi^{N}\right)=u^{N} g^{\prime}\left(\pi^{N}\right) \pi^{N} \\
& C^{k}\left(\pi^{k}, a^{k+1}\right)=u^{k}\left(a^{k+1}\right) g^{\prime}\left(\pi^{k}\right) \pi^{k}+\left(c^{k}\left(a^{k+1}\right)\right)^{\prime} \pi^{k} \\
& k \in\{0, \ldots, N-1\} .
\end{aligned}
$$


In the above equation, the $g\left(\pi^{k}\right)$ denotes the $S$-dimensional estimation error vector:

$$
g\left(\pi^{k}\right)=\left[\left\|e_{1}-\pi^{k}\right\|_{D},\left\|e_{2}-\pi^{k}\right\|_{D}, \cdots,\left\|e_{S}-\pi^{k}\right\|_{D}\right]
$$

\subsubsection{Solving Continuous Authentication Problem}

\section{Dynamic Programming}

In order to calculate the equation (4.8) effectively, we will use dynamic programming to compute the optimal policy. In other words, compute this equation backward from time $T$ to time 0 . The value function (4.8) can be rewritten as:

$$
J^{N}(\pi)=C^{N}(\pi)
$$

and for $k=N-1, N-2, \ldots, 0$

$$
\begin{aligned}
J^{k}(\pi)= & \min _{a^{k+1} \in\{1, \ldots, L\}}\left[C^{k}\left(\pi, a^{k+1}\right)\right. \\
& +\sum_{m=1}^{M_{a} k+1} J^{k+1}\left(\frac{B\left(a^{k+1}, O_{m}\left(a^{k+1}\right)\right) P^{\prime} \pi}{1_{S}^{\prime} B\left(a^{k+1}, O_{m}\left(a^{k+1}\right)\right) P^{\prime} \pi}\right) \\
& \left.\times 1_{S}^{\prime} B\left(a^{k+1}, O_{m}\left(a^{k+1}\right)\right) P^{\prime} \pi\right] \\
& \text { for all } \pi \in F .
\end{aligned}
$$

The optimal finite horizon value function of standard POMDP problem is piecewise linear and convex (PWLC), which was proved by Sondik [19] and Cassandra [20]. The value function of infinite horizon POMDP is not always PWLC, but it can be approximated with the value function of a large enough finite horizon POMDP. The piecewise theory is useful since the value function can be represented by a finite set 
of vectors such as:

$$
J^{k}(\pi)=\min _{i \in \Gamma^{k}}\left(\gamma_{i}^{k}\right)^{\prime} \pi \text { for all } \pi \in F
$$

Where $\Gamma^{k}$ is a finite set of $S$-dimensional vectors $\gamma_{i}^{k}$.

\section{Piecewise Linear Cost}

In our case, from the equation (4.9), $C^{k}\left(\pi^{k}, a^{k+1}\right)=u^{k}\left(a^{k+1}\right) g^{\prime}\left(\pi^{k}\right) \pi^{k}+\left(c^{k}\left(a^{k+1}\right)\right)^{\prime} \pi^{k}$, $g^{\prime}(\pi) \pi$ is $l_{2}$ norm estimation error, not a linear function of $\pi$, which makes our problem different from the standard POMDP problems. Fortunately V. Krishnamurthy [26] has showed that this estimation error can be approximated uniformly and arbitrarily closely with piecewise linear costs:

$$
g^{\prime}(\pi) \pi=\min _{r \in 1,2, \ldots, R} g_{r}^{\prime} \pi
$$

Where $R$ denotes the number of the $S$-dimensional vectors those are used to approximate the estimation error. With this approximation, our biosensor scheduling problem turns into a standard POMDP problem. All of the algorithms used to solve standard POMDP can be used in our case.

In [26], the nonlinear term $l_{2}$ state estimation cost is expressed as:

$$
g^{\prime}(\pi) \pi=1-\pi^{\prime} \pi
$$

and there are two modes to approximate the estimation error: lower-bound using Freudenthal triangularization and upper-bound using tangents [27]. The relationships among them are shown in the Figure 4.3, and the quadratic cost is convex which is showed in [26]. In our simulations, the upper bound approximation is used. The $g_{r}$ 


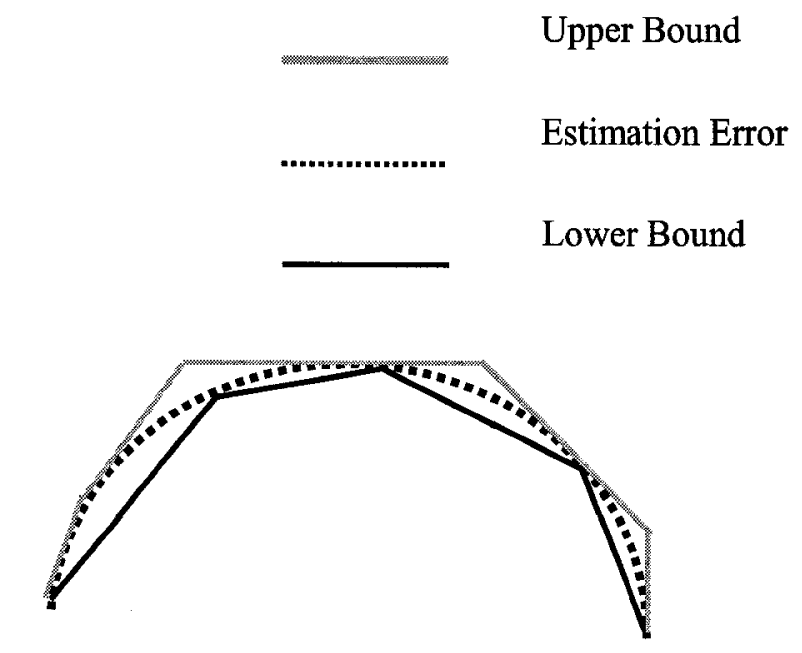

Fig. 4.3 Estimation error and its upper bound, lower bound approximations.

in equation (4.13) can be approximated by the tangents linear segment and the proof is shown as below:

$$
g_{r}=\left(1+\pi_{r}^{\prime} \pi_{r}\right) 1_{S}-2 \pi_{r}, \quad r=1,2, \ldots, R
$$

\section{Proof:}

Consider the hyperplane surface given by

$$
T=f\left(\pi_{1}, \pi_{2}, \ldots, \pi_{S}\right)=1-\pi^{\prime} \pi, 1_{S} \pi_{i}=1, i=1,2, \ldots, S
$$

and let $\pi_{r}=\left(\pi_{r, 1}, \pi_{r, 2}, \ldots, \pi_{r, S}\right)^{\prime}$ be any point on this surface, since this surface is strictly concave [26], then the $f\left(\pi_{1}, \pi_{2}, \ldots, \pi_{S}\right)$ is differentiable at point $\left(\pi_{r, 1}, \pi_{r, 2}, \ldots, \pi_{r, S}\right)^{\prime}$. The surface has a tangent plane at $\left(\pi_{r, 1}, \pi_{r, 2}, \ldots, \pi_{r, S}, T_{r}\right)^{\prime}$. The equation of the tangent plane at $\left(\pi_{r, 1}, \pi_{r, 2}, \ldots, \pi_{r, S}, T_{r}\right)^{\prime}$ is given as follows:

$$
f_{\pi_{1}}\left(\pi_{r, 1}, \pi_{r, 2}, \ldots, \pi_{r, S}\right)\left(\pi_{1}-\pi_{r, 1}\right)+f_{\pi_{2}}\left(\pi_{r, 1}, \pi_{r, 2}, \ldots, \pi_{r, S}\right)\left(\pi_{2}-\pi_{r, 2}\right)+\ldots
$$




$$
+f_{\pi_{S}}\left(\pi_{r, 1}, \pi_{r, 2}, \ldots, \pi_{r, S}\right)\left(\pi_{S}-\pi_{r, S}\right)+\left(T-T_{r}\right)=0
$$

where $f_{\pi_{i}}\left(\pi_{r, 1}, \pi_{r, 2}, \ldots, \pi_{r, S}\right)$ is the partial derivative of $f$ with respect to $\pi_{i}$ at point $\pi_{r}$.

Since $f\left(\pi_{1}, \pi_{2}, \ldots, \pi_{S}\right)=1-\pi^{\prime} \pi$, then

$$
f_{\pi_{i}}\left(\pi_{r, 1}, \pi_{r, 2}, \ldots, \pi_{r, S}\right)=2 \pi_{r, i}
$$

Substitute equation (4.17) into equation (4.16), we can get:

$$
\begin{aligned}
2 \pi_{r, 1}\left(\pi_{1}-\pi_{r, 1}\right) & +2 \pi_{r, 2}\left(\pi_{2}-\pi_{r, 2}\right)+\ldots \\
& +2 \pi_{r, S}\left(\pi_{S}-\pi_{r, S}\right)+\left(T-T_{r}\right)=0
\end{aligned}
$$

On this tangent surface,

for point $\pi=\left(1,0_{S-1}^{\prime}\right)^{\prime}$,

$$
T_{1}=T_{r}+2 \pi_{r, 1}^{2}+2 \pi_{r, 2}^{2}+\ldots+2 \pi_{r, S}^{2}-2 \pi_{r, 1},
$$

for point $\pi=\left(0,1,0_{S-2}^{\prime}\right)^{\prime}$,

$$
T_{2}=T_{r}+2 \pi_{r, 1}^{2}+2 \pi_{r, 2}^{2}+\ldots+2 \pi_{r, S}^{2}-2 \pi_{r, 2}
$$

and for point $\pi=\left(0_{S-1}^{\prime}, 1\right)^{\prime}$,

$$
T_{S}=T_{r}+2 \pi_{r, 1}^{2}+2 \pi_{r, 2}^{2}+\ldots+2 \pi_{r, S}^{2}-2 \pi_{r, S}
$$

Since $T_{r}=1-\pi_{r, 1}^{2}-\pi_{r, 2}^{2}-\ldots-\pi_{r, S}^{2}$, then 
for point $\pi=\left(1,0_{S-1}^{\prime}\right)^{\prime}$,

$$
T_{1}=1+\pi_{r, 1}^{2}+\pi_{r, 2}^{2}+\ldots+\pi_{r, S}^{2}-2 \pi_{r, 1}
$$

for point $\pi=\left(0,1,0_{S-2}^{\prime}\right)^{\prime}$,

$$
T_{2}=1+\pi_{r, 1}^{2}+\pi_{r, 2}^{2}+\ldots+\pi_{r, S}^{2}-2 \pi_{r, 2}
$$

and for point $\pi=\left(0_{S-1}^{\prime}, 1\right)^{\prime}$,

$$
T_{S}=1+\pi_{r, 1}^{2}+\pi_{r, 2}^{2}+\ldots+\pi_{r, S}^{2}-2 \pi_{r, S}
$$

And for any point $\pi=\left(\pi_{1}, \pi_{2}, \ldots, \pi_{S}\right)^{\prime}, 1_{S}^{\prime} \pi=1$ in information space, the value on the tangent plane can be expressed as:

$$
T=T_{1} \pi_{1}+T_{2} \pi_{2}+\ldots+T_{S} \pi_{S}=\left(\begin{array}{llll}
T_{1} & T_{2} & \cdots & T_{S}
\end{array}\right)\left(\begin{array}{c}
\pi_{1} \\
\pi_{2} \\
\vdots \\
\pi_{S}
\end{array}\right)
$$

Substitute equation (4.22), equation (4.23), equation (4.24) into equation (4.25), we get:

$$
T=\left(\left(1+\pi_{r}^{\prime} \pi_{r}\right) 1_{S}-2 \pi_{r}\right)^{\prime} \pi
$$

Therefore,

$$
g(\pi)^{\prime} \pi=\left(\left(1+\pi_{r}^{\prime} \pi_{r}\right) 1_{S}-2 \pi_{r}\right)^{\prime} \pi
$$




$$
g_{r}=\left(1+\pi_{r}^{\prime} \pi_{r}\right) 1_{S}-2 \pi_{r}, \quad r=1,2, \ldots, R
$$

\section{Optimal Algorithm}

As we described in Chapter 3, there are several algorithms to solve finite horizon POMDP such as Sondik's algorithm [19], incremental pruning, Cheng's linear support algorithm, the Witness algorithm and etc. The detail explanation and correspondent programming codes of these algorithms are presented in [24]. Any algorithm has the same basic framework and the only difference is the ways to compute a single dynamic programming step. The code of incremental pruning algorithm from [24] will be used in our examples. The desired solutions to POMDP are represented by a set of vectors, together with the optimal actions, and value function can be rewritten as:

$$
J^{k}(\pi)=\min _{i \in \Gamma^{k}}\left(\gamma_{i}^{k, *}\right)^{\prime} a_{i}^{k, *} \pi \text { for all } \pi \in F, \text { and } * \text { means optimal. }
$$

From this equation, each vector $\gamma$ is connected with an optimal sensor, a specific sensor for our problem. Therefore, we can solve our problem with two steps:

- Run off-line dynamic programming: Using any POMDP algorithm to compute the $\Gamma^{k}=\gamma_{i}^{k, *}$ together with the optimal biosensors $a_{i}^{k, *}$, where $i \in$ $1,2, \ldots,\left|\Gamma^{k}\right|$

- Run real time scheduling: Find the $\gamma_{i}^{k, *}$ for specific information state $\pi^{k}$ through equation (4.12), then the optimal biosensor is selected since each vector is connected with an optimal biosensor.

\subsubsection{Scheduling Algorithm with Security Requirement Constraint}

Different devices may have different security requirements. For some devices, it is desirable to guarantee FRR and FAR. In our formulation, the security requirement 
constraint is directly related to device security state estimation error. If the estimation error incurred by some biosensors exceeds the threshold, other biosensors with higher accuracy will be picked instead. Here we only consider local-in-time constraints (short term constraints) other than global constraints (long term constraints). The estimation error is specified as the expected estimation error. Our aim is to minimize the sensor usage cost subject to quadratic constraint on the expected estimation error. It is defined as:

$$
J_{a}=\min _{a} E\left\{\sum_{k=0}^{N-1}\left(c^{k}\left(a^{k+1}\right)\right)^{\prime} \pi^{k}\right\}
$$

subject to:

$$
\begin{array}{cc}
\sum_{m=1}^{M_{a}} \quad & u^{k+1}(l)\left(1-\frac{\pi^{\prime} P B^{2}\left(a, O_{m}(a)\right) P^{\prime} \pi}{\left(1_{S}^{\prime} B\left(a, O_{m}(a)\right) P^{\prime} \pi\right)^{2}}\right) \\
& \times\left(1_{S}^{\prime} B\left(a, O_{m}(a)\right) P^{\prime} \pi\right)<K_{l}, l \in \zeta_{c},
\end{array}
$$

where $\zeta_{c}$ denotes the set of sensors with constraints, $\zeta_{\bar{c}}$ denotes the set of sensors without constraints. and $\zeta=\{1, \ldots, L\}=\left\{\zeta_{c} \cup \zeta_{\bar{c}}\right\}$. Therefore, the problem with security requirement constraint can be solved in the following steps:

- Run off-line dynamic programming with action set $\zeta$ : Run this program with action set $\zeta$ to get the vectors $\gamma_{i}^{k, \zeta}$ and associated optimal biosensors $a_{i}^{k, \zeta, *}$.

- Run off-line dynamic programming with action set $\zeta_{\bar{c}}$ : Run this program with action set $\zeta_{\bar{c}}$ to get the vectors $\gamma_{i}^{k, \zeta_{\bar{c}}}$ and associated optimal biosensors $a_{i}^{k, \zeta \bar{c}, *}$.

- Run real time scheduling: Find the $\gamma_{i}^{k, \zeta}$ and $\gamma_{i}^{k, \zeta \bar{c}}$ for specific information state $\pi^{k}$ through equation (4.12). 
If $\pi^{k}$ satisfies equation (4.29), then biosensor $a_{i}^{k, \zeta, *}$ associated with vector $\gamma_{i}^{k, \zeta}$ will be chosen. Otherwise, biosensor $a_{i}^{k, \zeta_{\bar{c}}, *}$ associated with vector $\gamma_{i}^{k, \zeta_{\bar{c}}}$ will be selected instead.

\subsubsection{Scheduling Algorithm with System Resource Constraint}

Continuous authentication may consume extensive system resources. System resource constraints are important issues in MANETs. For example, the total energy available to the node is a scarce resource. Therefore, the total number of times using particular biosensor is constraint. For simplicity of the presentation, we assume that there are only constraints on the usage of biosensor 1: for $N$ horizon problem, biosensor 1 can only be used at most $N_{1}$ times.

Let $S_{1}=\left\{f_{1}, \ldots, f_{N_{1}+1}\right\}$ denote the set of $N_{1}+1$ dimensional unit vectors, where $f_{i}$ has 1 in the $i$ th position. We use process $z^{k}$ to denote the number of times biosensor 1 is used. Let $z^{k}$ be a $N_{1}+1$ state Markov chain with state space $S_{1}$. Let $z^{k}=f_{i}$ if biosensor 1 has been used $i-1$ times. The dynamics of $z^{k}$ are as follows. If biosensor 1 is used (i.e., $a^{k}=1$ ), $z^{k}$ jumps to state $f_{i+1}$. If any of other biosensors is used, $z^{k}$ remains unchanged. We can express $z^{k}$ as a deterministic Markov chain with dynamics given by

$$
z^{k}=Q^{\prime}\left(a^{k}\right) z^{k-1}, \quad z^{0}=e_{1}, z^{N}=e_{N+1}
$$


where the transition probability matrix $Q(\cdot)$ is defined as

$$
Q\left(a^{k}=1\right)=\left(\begin{array}{ccccc}
0 & 1 & 0 & \cdots & 0 \\
0 & 0 & 1 & \cdots & 0 \\
\vdots & \vdots & \vdots & \ddots & 1 \\
0 & 0 & 0 & \cdots & 1
\end{array}\right)
$$

and $Q\left(a^{k}\right)=I_{\left(N_{1}+1\right) \times\left(N_{1}+1\right)}$ if $a^{k} \neq 1$.

In order to use equation (4.11) to get the optimal biosensor scheduling policy with resource constraints, we can make the following coordinate exchange. Consider the augmented Markov chain $\left(X^{k}, z^{k}\right)$, which has transition probability matrix $\bar{P}=P \otimes Q$, information state of $\left(X^{k}, z^{k}\right)$ is $\bar{\pi}^{k}=\pi^{k} \otimes z^{k}$ and observation probability matrix $\bar{B}\left(a, O_{m}(a)\right)=B\left(a, O_{m}(a)\right) \otimes I_{N+1}$, where $\otimes$ denotes tensor (Kronecker product). Thus, the augmented information state $\bar{\pi}^{k}$ evolves according to the standard HMM filter with $P, B$ replaced by $\bar{P}, \bar{B}$. Define the value function

$$
\bar{J}^{k}=J^{k}(\pi, z), \bar{\pi}=\pi \otimes z
$$

Now we can use equation (4.11) to solve the above value function by substituting $J^{k}$ with $\bar{J}^{k}, \pi^{k}$ with $\bar{\pi}^{k}, P$ with $\bar{P}$, and $B$ with $\bar{B}$. 


\subsection{User to Network Authentication}

MANET has no fixed infrastructure and allows the nodes to change frequently. In such a self-organized network, each node can pass information and control packets from one neighbor node to another. This type of network is very useful in coalition operation in war fields where exchanging information is needed between soldiers, commanders, and allies. The security of this type of network is guaranteed only if the identities of the all the nodes can be verified. Authentication provides a way to confirm the identity claimed by the user, and any one of biometric-based authentication is sufficient to directly check an authorized user.

The type of user authentication is classified into either user-to-device or user-tonetwork authentication and the difference is the location of the biometric templates. In general, the user-to-network authentication is used to authenticate a user in wireless application while the user-to-device authentication is used to build the trust between a user and his device. The continuous authentication scheme we previously introduced is used to address the user-to-device authentication problem, which can be used to lock and unlock the device. But once the device is compromised, this device can be used by the intruder to access the network. Therefore, the user-to-network authentication is necessary in MANETs. However, there is no centralized server available in MANETs, biometric-based user-to-network authentication becomes a challenge. Here a new scheme is proposed to address the needs of biometric authentication for information security in MANETs and services.

\subsubsection{Decentralized Authentication Scheme}

Take coalition cooperation as an example. We assume that each soldier is equipped with a mobile device, which stores a personal profile that consists of personal ID 


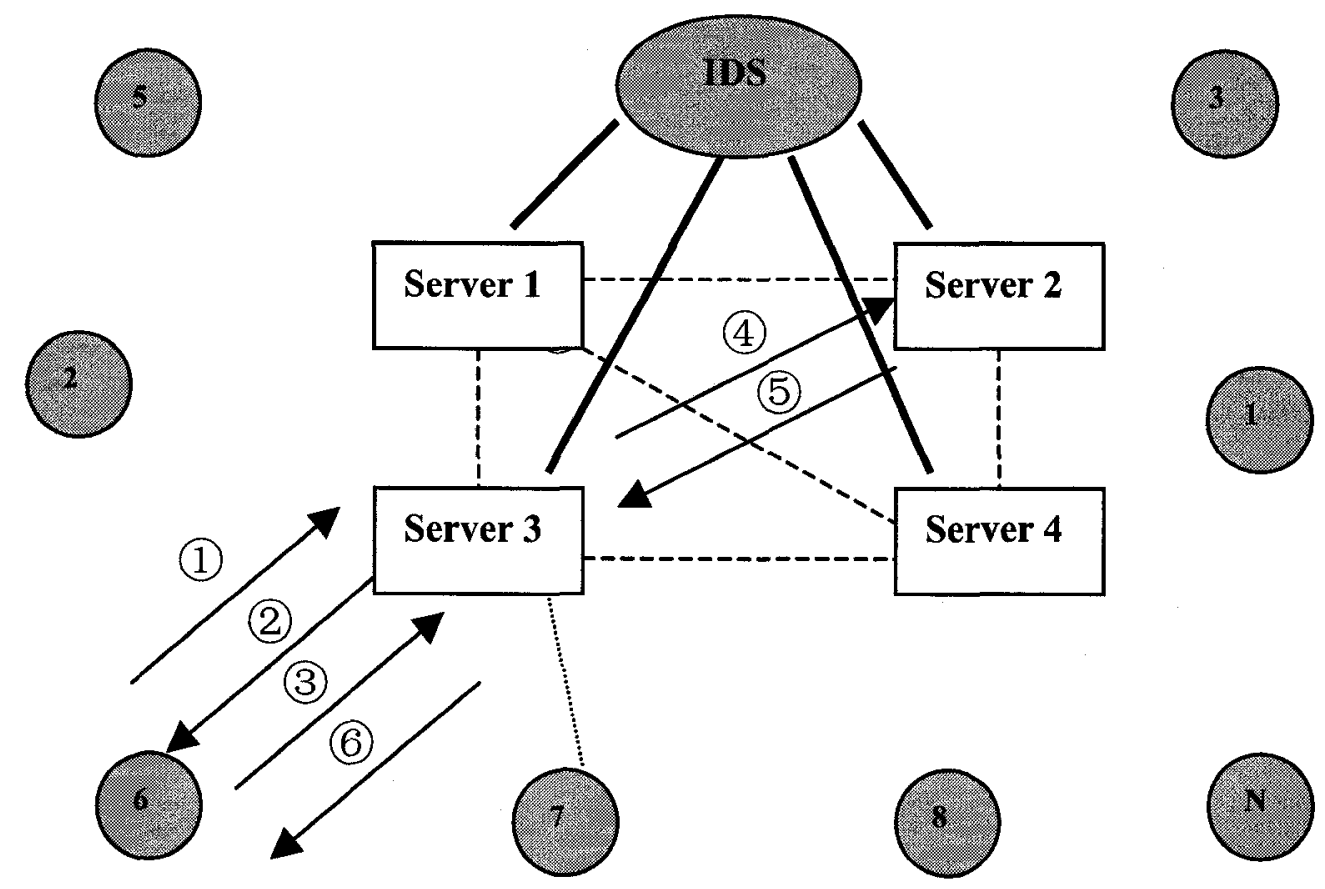

(1) Node 6 broadcasts the request to join the network.

(2) Server 3 receives this message, and requires node 6 to provide its identification.

(3) Node 6 sends its fingerprint to server 3.

(4) Server 3 checks its record and knows that server 2 is responsible for authentication. Server 3 sends the fingerprint to Server 2.

(5) Server 2 authenticates node 6 , and sends the authentication result back to Server 3.

(6) Server 3 allows or rejects the node 6 to join the network.

Fig. 4.4 User authentication procedures. 
and some other information to help identification. Each mobile device is equipped with a biometric sensor (or some biometric sensors used for multi-modal biometric authentication). We can apply more complicated but more accurate biometrics to user-to-network authentication. For the group leader, it is reasonable to assume that he/she is equipped with one powerful device (or a platform) that has strong computation capability, big storage and long battery life. This device (we call it server in this thesis) can be used to store all of the templates and conduct computations. Instead of a centralized authentication server, the group leaders act as distributed authentication servers, and there is one and only one server that will be responsible for authentication at each time instant. From now on, we call the server that acts as authentication server as active server, the other servers as passive servers.

A decentralized algorithm can be designed to decide which server will take this responsibility automatically. The cryptography key is used for communication between the servers. Since the servers storing the soldier templates are the critical points in MANETs, changing the authentication server with time improves the system security. The authentication procedures are illustrated in Figure 4.4 and operated as follows:

1. When a solider wants to join current network, he broadcasts a request message. This message consists of his personal ID. When a group leader (a server) receives this message, if he is currently responsible for authentication, he will ask the solider to send his biometrics. Once the authentication is successful, the soldier is allowed to join the network.

2. If the group leader (the server) is not currently responsible for network authentication, he will check his record and find which server is the authentication server. Then he requires the biometrics from the soldier and forwards this to the authentication server. The authentication result will be sent back to the access 
server and the soldier can join the network if the authentication is successful. If two or more servers receive the requests from the solider, the authentication server will select one server to send back the authentication result.

3. We can continuously authenticate the devices. The re-authentication procedure is similar to the first time authentication procedure described above.

\subsubsection{Authentication Server Scheduling Formulation}

In our proposed scheme, group leaders (or servers) are the critical points in MANETs since they store the soldier templates and are responsible for authentication. When a server acts as the authentication server, more traffic will flow to it compared to other passive servers, and accordingly, it is easier to be detected by the enemy. We assume that each server can take some countermeasures to decrease the information exposure to the enemy. The cumulative information emission radiated from the authentication server and detected by the enemy directly affects the threat posted to the servers. We assume that this threat level posed to a server can be indirectly measured by the IDS in MANETs and any server can access the IDS at any time instant. In order to improve the security of these servers, we present decentralized algorithms to reduce the detectability of servers to the enemy, in other words, we control the information emission to the enemy by scheduling the authentication servers. Motivated by [28], we use multi-armed bandit algorithms to dynamically manage and control the emission of multiple servers in combat situations. With multi-armed bandits algorithms, the optimal server scheduling policy can be found by a so-called Gittins index rule [29]. That means at each time instant, it is optimal to use the server with the highest Gittins index as the authentication server to minimize the overall threat level to all servers. Since the threat level is not directly available to our system, we use the value 
acquired from the IDS as the observation of the threat level, which is a probabilistic function of the unobserved finite state Markov chain.

\subsubsection{Multi-armed Bandit Problem}

Consider $L$ independent servers, enumerated by $l=1,2, \ldots, L$. Assume each server has a finite number of $N_{l}$ states. Let $s^{k}(l)$ denote the emission level of the server $l$ at the time instant $1,2, \ldots, k . s^{k}(l)$ is modeled as a Markov chain. At each time instant, there is only one server acts as the authentication server. If server $l$ works at time instant $k$, an instantaneous reward $\beta^{k} R\left(s^{k}(l), l\right)$ is accrued (in our case it is cost and is a negative value), and $R\left(s^{k}(l), l\right) \geq 0$ is assumed finite (if any $C(i, l)$ is negative, we simply set $R(i, l)=-C(i, l))$. Here $0 \leq \beta \leq 1$ denotes the discount factor. The state $s^{k}(l)$ evolves according to an $N_{l}$-state Markov chain with transition probability matrix:

$$
P(l)=\left(a_{i j}\right)_{i, j \in N_{l}}=\operatorname{Pr}\left(s^{k+1}(l)=j \mid s^{k}(l)=i\right)
$$

if server $l$ works as the authentication server at time $k$. The states of all other servers $L-1$ are unaffected. That is, $s^{k+1}(l)=s^{k}(l)$, if server $l$ is not authentication server at time instant $k$.

The threat level of the authentication server can be indirectly observed by the output $y^{k+1}(l)$ of IDS when the authentication server stays in state $s^{k+1}(l)$. Assume that there is a finite $M_{l}$ observation set indexed by $m(l)=1,2, \ldots, M_{l}$. We define $Y^{k}=\left(y^{1}\left(a^{0}\right), \ldots, y^{k}\left(a^{k-1}\right)\right)$ as the observation history for time instant $k$. Let $B(l)=$ $\left(b_{i m}(l)\right)_{i \in N_{l}, m \in M_{l}}$ denote the observation probability matrix of the HMM, where each 
element

$$
b_{i m}(l)=\operatorname{Pr}\left(y^{k+1}(l)=m \mid s^{k+1}(l)=i, a^{k}=l\right),
$$

and $a^{k} \in\{1,2, \ldots, L\}$ denotes that server $l$ is the authentication server at time instant $k$. Let the policy $a$ denote the server sequence as $\left\{a^{k}, k=1,2, \ldots, k\right\}$. The total expected discounted reward over an infinite time horizon is defined as:

$$
J_{a}=E\left[\sum_{k=0}^{\infty} \beta^{k} R\left(s^{k}\left(a^{k}\right), a^{k}\right)\right] .
$$

The aim is to find the optimal policy that produces the maximum reward.

For each server $l, x^{k}(l)$ is the information state at time $k$ and is denoted as:

$$
x^{k}(l)=\left(x_{i}^{k}(l)\right), i=1,2, \ldots, N_{l}, x_{i}^{k}(l)=\operatorname{Pr}\left(s^{k}(l)=i \mid Y^{k}, a^{k-1}=l\right)
$$

where $Y^{k}$ and $a^{k-1}$ are observation history and server which works on time instant $k$. Let $\chi(l)$ denote the state space of information states $x_{i}(l)$, which is a $l-1$ dimension simplex, denoted as:

$$
\chi(l)=\left\{x(l) \in \Re^{N_{l}}: 1_{N_{l}}^{\prime} x(l)=1,0 \leq x_{i}(l) \leq 1, \text { for all } i \in 1, \ldots, N_{l}\right\} .
$$

With the new observation $y^{k+1}(l)$, the information state $x^{k}(l)$ can be recursively updated by the HMM state filter that is known as forward algorithm. If server $l$ is the authentication server at time instant $k$,

$$
x^{k+1}(l)=\frac{B\left(l, y^{k+1}(l)\right) P^{\prime}(l) x^{k}(l)}{1_{N_{l}}^{\prime} B\left(l, y^{k+1}(l)\right) P^{\prime}(l) x^{k}(l)},
$$


where if $y^{k+1}(l)=m$, then

$$
B(l, m)=\left(\begin{array}{cccc}
b_{1 m}(l) & \ldots & 0 & 0 \\
0 & b_{2 m}(l) & \ldots & 0 \\
\vdots & 0 & \ddots & 0 \\
0 & 0 & \cdots & b_{N_{l} m}(l)
\end{array}\right)
$$

The states of the other servers (passive servers) keep unaffected:

$$
x^{k+1}(q)=x^{k}(q) \text {, if server } q \text { is not the authentication server, } q \in\{1, \ldots, L\}, q \neq l \text {. }
$$

We denote the reward $R_{a^{k}}$ as $N_{a^{k}}$ dimensional vector $R\left(a^{k}\right)$ :

$$
\left.\left[R\left(s^{k}(l)=1, a^{k}\right)\right), \ldots, R\left(s^{k}(l)=N_{a^{k}}, a^{k}\right)\right]^{\prime}
$$

Substitute (4.38) into (4.33), the total expected discounted reward over an infinite time horizon is expressed as:

$$
J_{a}=E\left[\sum_{k=0}^{\infty} \beta^{k} R^{\prime}\left(a^{k}\right) x^{k}\left(a^{k}\right)\right]
$$

The optimal policy has an index rule: For each server, there is a function $\gamma^{k}\left(l, x^{k}(l)\right)$ called Gittins index, which is the function of server $l$ and its information state $x^{k}(l)$. The server with the biggest Gittins index at time instant $k$ acts as the authentication server, i.e.,

$$
\text { Activate server } q \text { where } q=\max _{l \in 1, \ldots, L} \gamma\left(l, x^{k}(l)\right) \text {. }
$$

Thus, computing Gittins index is the key requirement to solve multi-armed bandits 
(multi-server scheduling) problem.

\subsubsection{Value Iteration Algorithm for Computing Gittins Index}

The Gittins index for each server $l$ can be solved with dynamic programming formulation, and this computation is off-line, independent of the other $L-1$ servers.

For each server $l$, let $M(l)$ denote a positive real number such that

$$
0 \leq M(l) \leq \bar{M}(l), \bar{M}(l)=\max _{i \in N_{l}} \frac{R\left(s^{k}(l)=i, a^{k}=l\right)}{1-\beta}
$$

We omit the $l$ in $M(l)$ and $\bar{M}(l)$ and the superscript $k$ in $x^{k}(l)$ for simplification, the Gittins index of server $l$ with information state $x(l)$ can be defined as:

$$
\gamma(l, x(l))=\min \left\{M: V^{l}(x(l), M)=M\right\}
$$

where $V^{l}(x(l), M)$ is the value function for server $l$ and satisfies the functional Bellman's recursion

$$
\begin{aligned}
& V^{l}(x(l), M)=\max \left\{R^{\prime}(l) x(l)\right. \\
& \left.+\beta \sum_{m=1}^{M_{l}} V^{l}\left(\frac{B(l, m) P^{\prime}(l) x(l)}{1_{N_{l}}^{\prime} B(l, m) P^{\prime}(l) x(l)}, M\right) 1_{N_{l}}^{\prime} B(l, m) P^{\prime}(l) x(l), M\right\}
\end{aligned}
$$

where $M$ denotes the parameterized retirement reward.

For the finite time horizon $N$, the value iteration algorithm $k=0, \ldots, N-1$ :

$$
\begin{aligned}
& V^{l, k+1}(x(l), M)=\max \left\{R^{\prime}(l) x(l)\right. \\
& \left.+\beta \sum_{m=1}^{M_{l}} V^{l, k}\left(\frac{B(l, m) P^{\prime}(l) x(l)}{1_{N_{l}}^{\prime} B(l, m) P^{\prime}(l) x(l)}, M\right) 1_{N_{l}}^{\prime} B(l, m) P^{\prime}(l) x(l), M\right\}
\end{aligned}
$$

where $V^{N}(x(l), M)$ is the value function of an $N$-horizon dynamic programming 
recursion. Let $\gamma^{N}(l, x(l))$ denote the approximate Gittins index computed via (4.44), i.e.,

$$
\gamma^{N}(l, x(l))=\min \left\{M: V^{l, N}(x(l), M)=M\right\}
$$

The finite horizon Gittins index can be made arbitrarily accurate by choosing the horizon $N$ large enough [29].

\subsubsection{Finite Dimensional Characterization of Gittins Index}

The value iteration recursion (4.44) cannot be solved with practical methodologies. But it can be translated into a standard POMDP value function with a different coordinate basis [29]. Since the value function of standard POMDP is piecewise linear and convex, the Gittins index can be computed with these piecewise linear segments.

In order to find a solution of computing Gittins index, a fictitious retirement information state is added. Once the information state reaches this value, it remains there for all time and accruing no reward. Define the $\left(N_{l}+1\right)$ dimensional augmented information state

$$
\bar{x} \in\left\{\left[x^{\prime}, 0\right]^{\prime},\left[0_{N_{l}}^{\prime}, 1\right]^{\prime}\right\}, \quad x \in \chi(l),
$$

where $\bar{x}^{k}=\left[0_{N_{l}}^{\prime}, 1\right]^{\prime}$ denotes the retirement information state. The augmented observation process is defined as $y^{k} \in\left\{1, \ldots, M_{l}+1\right\}$, and the observation $M_{l}+1$ corresponds to a fictitious observation which causes the information state jumps into the retirement state. The $\left(N_{l}+1\right) \times\left(N_{l}+1\right)$ dimensional transition matrix observation 
probability matrices are defined as:

$$
\begin{aligned}
& P_{1}(l)=\left(\begin{array}{cc}
P(l) & 0_{N_{l}} \\
0_{N_{l}}^{\prime} & 1
\end{array}\right), \\
& B_{1}(l)=\left(\begin{array}{cc}
B(l) & 0_{N_{l}} \\
0_{N_{l}}^{\prime} & 1
\end{array}\right), \\
& P_{2}(l)=\left(\begin{array}{cc}
0_{N_{l} \times N_{l}} & 1_{N_{l}} \\
0_{N_{l}}^{\prime} & 1
\end{array}\right), \\
& B_{2}(l)=I_{\left(N_{l}+1\right) \times\left(N_{l}+1\right)}, \\
& B_{1}(l, m)=\operatorname{diag}\left(\operatorname{column} m \text { of } B_{1}(l)\right), \\
& B_{2}(l, m)=\operatorname{diag}\left(\operatorname{column} m \text { of } B_{2}(l)\right), m \in\left\{1, \ldots, M_{l}+1\right\} .
\end{aligned}
$$

We will use coordinate transformation to construct a standard POMDP, and its value function is $V^{l}(x(l), M)$. Since $0 \leq M \leq \bar{M}$, define the pseudo-information state

$$
z=\left(\begin{array}{c}
M / \bar{M} \\
1-M / \bar{M}
\end{array}\right), 0 \leq M \leq \bar{M} \text {. }
$$

Define the information state $\pi$ and following coordinate transformation (where $\otimes$ denotes Kronecker product),

$$
\begin{aligned}
& \pi=z \otimes \bar{x}, \\
& \bar{P}_{1}=I_{2 \times 2} \otimes P_{1}=\left(\begin{array}{cc}
P_{1} & 0 \\
0 & P_{1}
\end{array}\right), \\
& \bar{P}_{2}=I_{2 \times 2} \otimes P_{2}=\left(\begin{array}{cc}
P_{2} & 0 \\
0 & P_{2}
\end{array}\right), \\
& \bar{B}_{1}(l, m)=I_{2 \times 2} \otimes B_{1}(l, m),
\end{aligned}
$$




$$
\begin{aligned}
& \bar{B}_{2}(l, m)=I_{2 \times 2} \otimes B_{2}(l, m), \\
& \bar{R}_{1}(l)=\left(\begin{array}{llll}
R^{\prime}(l) & 0 & R^{\prime}(l) & 0
\end{array}\right)^{\prime} \\
& \bar{R}_{2}(l)=\left(\begin{array}{llll}
\bar{M} 1_{N_{l}}^{\prime} & 0 & 0_{N_{l}}^{\prime} & 0
\end{array}\right)^{\prime} .
\end{aligned}
$$

Information state $\pi(l)$ is a $2\left(N_{l}+1\right)$ dimensional vector and belongs to $\Pi(l)$ where

$$
\Pi(l)=\left\{\pi: 1_{2\left(N_{l}+1\right)}^{\prime} \pi(l)=1, \text { and } \pi_{i}(l) \geq 0, i=1,2, \ldots, 2\left(N_{l}+1\right)\right\}
$$

Finially define the control variable $v^{k} \in\{1,2\}$ at each time instant, $v^{k}=1$ means continue and $v^{k}=2$ means retire.

With the definition of transition matrix $\bar{P}(l)$, observation matrix $\bar{B}(l)$, reward matrix $\vec{R}(l)$ of two valued control $\left(v^{k} \in\{1,2\}\right)$ in equation (4.49) and objective

$$
\max _{v} E\left[\sum_{k=0}^{N} \beta^{k} \bar{R}_{v^{k}}^{\prime}(l) \pi^{k}\right]
$$

we define a standard POMDP. Its information state $\pi(l)$ is updated according to

$$
\begin{array}{r}
\pi^{k+1}(l)=\frac{\bar{B}_{v^{k}}\left(l, \bar{y}^{k+1}\right)\left(\bar{P}_{v^{k}}(l)\right)^{\prime} \pi^{k}(l)}{1_{2\left(N_{l}+1\right)}^{\prime} \bar{B}_{v_{k}}\left(l, \bar{y}^{k+1}\right)\left(\bar{P}_{v_{k}}(l)\right)^{\prime} \pi^{k}(l)} \\
v^{k} \in\{1,2\}, \bar{y}^{k+1} \in\left\{1, \ldots, M_{l}+1\right\}
\end{array}
$$

The value iteration recursion for optimizing this POMDP over the finite horizon $N$ is given by

$$
\begin{aligned}
& \bar{V}^{k+1}(l, \pi(l))=\max \left\{\bar{R}_{1}^{\prime}(l) \pi(l)\right. \\
& +\beta \sum_{m=1}^{M_{l}+1} \bar{V}^{k}(l)\left(\frac{\bar{B}_{1}(l, m)\left(\bar{P}_{1}(l)\right)^{\prime} \pi^{k}(l)}{1^{\prime} \bar{B}_{1}(l, m)\left(\bar{P}_{1}(l)\right)^{\prime} \pi^{k}(l)}\right) 1^{\prime} \bar{B}_{1}(l, m)\left(\bar{P}_{1}(l)\right)^{\prime} \pi^{k}(l),
\end{aligned}
$$




$$
\begin{aligned}
& \left.\bar{R}_{2}^{\prime}(l) \pi(l)+\beta \sum_{m=1}^{M_{l}+1} \bar{V}^{k}(l)\left(\frac{\bar{B}_{2}(l, m)\left(\bar{P}_{2}(l)\right)^{\prime} \pi^{k}(l)}{1^{\prime} \bar{B}_{2}(l, m)\left(\bar{P}_{2}(l)\right)^{\prime} \pi^{k}(l)}\right) 1^{\prime} \bar{B}_{2}(l, m)\left(\bar{P}_{2}(l)\right)^{\prime} \pi^{k}(l)\right\}, \\
& k=1,2 \ldots, N, \\
& \bar{V}_{0}(l, \pi)=\max \left\{\bar{R}_{1}^{\prime}(l) \pi(l), \bar{R}_{2}^{\prime}(l) \pi(l)\right\} .
\end{aligned}
$$

Here $\bar{V}^{k}(l, \pi)$ denotes the value function of the dynamic program,

$$
\left.\bar{V}^{k}(l, \pi)=\max E\left[\sum_{t=N-k}^{N} \beta^{t} \bar{R}_{v^{t}}^{\prime}(l)\right) \pi^{t} \mid \pi^{N-k}=\pi\right]
$$

In [29], V. Krishnamurthy shows that the value function in equation (4.53) has several characteristics:

- The value function in equation (4.44) is equal to the value function of the standard POMDP defined in equation (4.53).

- The value function $\bar{V}^{k}(l, \pi(l))$ is piecewise linear and convex and has the finite dimensional representation:

$$
\bar{V}^{k}(l, \pi(l))=\max _{\lambda_{i}^{k} \in \Lambda^{k}(l)}\left(\lambda_{i}^{k}\right)^{\prime} \pi(l)
$$

where each $2\left(N_{l}+1\right)$-dimension vector $\lambda_{i}^{k}$ is of the form

$$
\lambda_{i}^{k}=\left(\begin{array}{llll}
\left(\lambda_{i}^{k}(1)\right)^{\prime} & 0 & \left(\lambda_{i}^{k}(3)\right)^{\prime} & 0
\end{array}\right)^{\prime}, \text { where } \lambda_{i}^{k}(1), \lambda_{i}^{k}(3) \in \Re^{N_{l}}
$$

If all the elements of $R(l)$ are not equal, then there always exists a unique vector in $\Lambda^{k}(l)$ which we denote by $\lambda_{1}^{k}=\left(\begin{array}{cc}\bar{M} 1_{N_{l}}^{\prime} & 0_{N_{l}+2}^{\prime}\end{array}\right)^{\prime}$ with optimal control $v^{k}=2$. If all the elements of $R(l)$ are equal, then $\Lambda^{k}(l)$ compromises of a single vector $\lambda_{1}^{k}=\left(\begin{array}{llll}\bar{M} 1_{N_{l}}^{\prime} & 0 & \bar{M} 1_{N_{l}}^{\prime} & 0\end{array}\right)^{\prime}$. 
- For any information state $x(l) \in \chi(l)$ of server $l$, the Gittins index $\gamma^{N}(l, x(l))$ is given by the finite dimensional representation

$$
\gamma^{N}(l, x(l))=\max _{\lambda_{i}^{N} \in \Lambda^{N}} \frac{\bar{M}\left(\lambda_{i}^{N}(3)\right)^{\prime} x(l)}{\left(\lambda_{i}^{N}(3)-\lambda_{i}^{N}(1)\right)^{\prime} x(l)+\bar{M}}
$$

Therefore, we can calculate the Gittins index of server $l$ by using the value function of standard POMDP defined in equation (4.53).

\subsubsection{Solving the Authentication Server Scheduling Problem}

The complete authentication server scheduling problem based on the multi-armed bandits theory is given as follows:

\section{Off-line Computation of Gittins Index}

1. Input for each server $l=1,2, \ldots, L: P(l)\{$ Transition probability matrix $\}$, $B(l)\{$ Observation probability matrix $\}, R(l)\{$ Reward vector $\}, x^{0}(l)\{$ A priori state estimate at time 0$\}, N\{$ Horizon length $\}$, and $\beta\{$ Discount factor $\}$, then off-line compute finite set of vectors $\Lambda^{N}(l)$.

2. At time $k=0$, compute $\gamma^{N}\left(l, x^{0}(l)\right)$ according to equation (4.57).

\section{Real-time Server Scheduling over Horizon $N$}

Assume that any time instant $k$, every server stores the $L$-dimensional vector $\left(a^{k}, \gamma\right)$, where $a^{k}$ denotes the authentication server, $\gamma$ is the vector of Gittins indices of the $L-1$ servers, arranged in descending order, i.e.,

$$
\gamma=\left(\gamma\left(1, x^{k}(1)\right), \gamma\left(2, x^{k}(2)\right), \ldots, \gamma\left(L-1, x^{k}(L-1)\right)\right)
$$


where $\gamma\left(1, x^{k}(1)\right)$ is the passive server that has the highest Gittins index, and $\gamma(L-$ $\left.1, x^{k}(L-1)\right)$ is the passive server with the lowest Gittins index.

Then the real-time steps for server scheduling are:

1. Let server $l=\max _{l \in 1, \ldots, L}\{\gamma(l, x(l))\}$ be the authentication server.

2. Obtain the threat level measurement $y^{k+1}(l)$ from IDS.

3. Update the threat level estimation of the lth server using the HMM filter (4.36).

4. Compute $\gamma^{N}\left(l, x^{k+1}(l)\right)$ of server $l$ according to equation (4.57).

5. For the other servers $q=1,2, \ldots, L, q \neq l$, the Gittins index keep unchanged $\gamma^{N}\left(q, x^{k+1}(q)\right)=\gamma^{N}\left(q, x^{k}(q)\right)$

6. If $\gamma^{N}\left(l, x^{k+1}(l)\right)>\gamma^{N}\left(1, x^{k}(1)\right)$, server $l$ will continue to be the same authentication server $l$. Else, if $\gamma^{N}\left(l, x^{k+1}(l)\right)<\gamma^{N}\left(1, x^{k}(1)\right)$, server $l$ will broadcast $\gamma^{N}\left(l, x^{k+1}(l)\right)$ and become passive server.

7. On receiving this broadcast, server 1 that has the highest Gittins index acts as the authentication server.

8. All servers update the vector $\left(a^{k+1}, \gamma\right)$ where now $\gamma^{N}\left(l, x^{k+1}(l)\right)$ is one of the elements of $\gamma$. 


\subsection{Summary}

User-to-device and user-to-network are two classes of user authentication in MANETs. Normally user-to-device authentication is used to lock and unlock devices by authenticating a user to a device, while user-to-network authentication provides a way to authenticate user in networked applications. When biometrics is used for authentication, the main difference between them is where the users' biometric templates are kept.

In this chapter, we first proposed a framework of continuous authentication in MANETS. In this framework, multimodal biometrics is used for continuous authentication, and we formulated the whole system as a partially observed Markov decision process. We used dynamic programming-based hidden Markov model scheduling algorithms to derive the optimal schemes for continuous authentication. The current proposed user-to-device authentication scheme can also satisfy the security requirement constraint and system resource constraint separately. Future research needs to be conducted to consider these two constraints together.

Then we proposed a new decentralized user-to-network authentication scheme in MANETs. In this new decentralized authentication scheme, the users' templates are stored in several servers, which are controlled by group leaders (commanders); The device carried by soldiers authenticates themselves by broadcasting requests. At each time instant, only one server acts as the authentication server, while the other servers keep passive, which means that they are only responsible for transmitting the message between the authentication server and devices. The servers also keep all the other servers' biometric templates, and they cryptographically communicate with each other.

There is an emission level connected to each server. The authentication server 
will emit some information to the enemy when it authenticates a device every time because of the traffic increase. The threat level will decrease if the server takes some countermeasures to protect itself. The IDS in MANETs provides the measurement of the threat level for each server. When the server acts as the authentication server, its emission lever involves as a POMDP process. The passive server's emission level keeps unchanged. In order to minimize the total emission level, we used multi-armed bandits theory to build an optimal policy to decide which server will act as the authentication server at a certain time. The basic idea is: Gittins index is computed for each server and the server with the highest Gittins index will act as the authentication server. The off-line and real-time algorithms were described in this chapter. Numerical examples will be given in Chapter 5 . 


\section{Chapter 5}

\section{Numerical Examples and}

\section{Simulation Results}

In this section, we illustrate the performance of the proposed schemes by simulations. Three scenarios are considered. The first scenario involves a MANET that uses iris biosensor for user-to-device continuous authentication. In this scenario, we model it as a two-state HMM problem with two sensors. The first one is an iris biosensor. For the other one, no sensor will be used, and we estimate the device security state by using the HMM state predictor. Here we call it prediction sensor.

The second scenario involves two biosensors (Iris biosensor and fingerprint biosensor) in the MANET. In this scenario, we can model it as a two-sate HMM problem with three sensors. The first sensor is an iris biosensor. The second one is a prediction sensor, which is the same as that used in scenario 1. The fingerprint is treated as the third sensor.

The third scenario involves two types of servers used in the decentralized authentication scheme. In this scenario, the multi-server scheduling problem is formulated into a two-state POMDP model with multi-armed bandits structure. Gittins indices 
are computed for each type of server.

All simulations are run on Redhat Linux: 3.0G CPU, 512M memory, and the Kernel version is $2.4 .20-31.9$.

\subsection{Scenario 1 - Optimal Continuous Authentication}

\subsubsection{Parameters}

State Space: The state space is the status of the device: $X_{1}=$ safe and $X_{2}=$ compromised. We use the following transition probability matrix of $X^{k}$ :

$$
P=\left(\begin{array}{ll}
0.7 & 0.3 \\
0.1 & 0.9
\end{array}\right)
$$

Here, we assume that the safe device could be compromised with probability 0.3 and the compromised device could be snatched back with low probability 0.1 .

Sensors: Assume that two sensors are used, i.e., $L=2$.

- Iris sensor: This iris biosensor acts as a HMM filter and yields the authentication result of the device.

- Prediction sensor: No sensor is used, and the state of the device is predicted. Here the prediction sensor is an HMM state predictor.

Observation Symbols: When using the iris biosensor, the observation symbols from the iris biosensor at each time $k$ consist of the result $O_{1}=$ safe or $O_{2}=$ compromised. Since the prediction sensor will incur nothing, we will add one more observation symbol $O_{3}=$ nothing to denote that no observation is made. We define $\hat{B}\left(a^{k}\right)=\left[\hat{B}_{i j}\left(a^{k}\right)\right]=P_{r}\left\{y^{k}\left(a^{k}\right)=O_{j} \mid X^{k}=e_{i}\right\}$. So we can assign the observation 
matrix $\hat{B}\left(a^{k}\right)$ as:

$$
\hat{B}\left(a^{k}=\text { iris }\right)=\left(\begin{array}{ccc}
0.9 & 0.1 & 0 \\
0.1 & 0.9 & 0
\end{array}\right),
$$

where we assume that $\mathrm{FRR}=\mathrm{FAR}=0.1$.

$$
\hat{B}\left(a^{k}=\text { prediction }\right)=\left(\begin{array}{ccc}
0 & 0 & 1 \\
0 & 0 & 1
\end{array}\right) .
$$

Cost Function: There are two components in the cost function. The first component is the sensor costs.

$$
\begin{aligned}
& c\left(X^{k}=e_{i}, a^{k+1}=\text { iris }\right)=\rho^{\text {iris }}+r^{\text {iris }} \\
& c\left(X^{k}=e_{i}, a^{k+1}=\text { prediction }\right)=\rho^{\text {prediction }}+r^{\text {prediction }} .
\end{aligned}
$$

We assume $\rho^{\text {iris }}=10, \rho^{\text {prediction }}=7$, which means that the cost of using the iris biosensor is higher than the prediction sensor. $r$ denotes the information leakage by using a sensor. For example, if the current information state is $[0.2,0.8]$, it means that we $80 \%$ believe that the device is compromised. In this situation, the sensor with higher accuracy will be preferred for re-authentication. Otherwise, FAR would be higher, and more information will be captured by the attackers. We use the following values.

$$
\begin{aligned}
& r^{\operatorname{iris}}\left(X^{k}=0\right)=0.5, r^{\text {iris }}\left(X^{k}=1\right)=2, \\
& r^{\text {predcition }}\left(X^{k}=0\right)=1.2, r^{\text {prediction }}\left(X^{k}=1\right)=5.2,
\end{aligned}
$$


where 0 means the safe state, and 1 means the compromised state.

Estimation error: For the $l_{2}$ norm estimation error,

$$
g^{\prime}\left(\pi^{k}\right) \pi^{k}=u^{k}\left[1-\left(\pi^{k}\right)^{\prime} \pi^{k}\right]
$$

where we use $u^{k}=3$ in our simulations. In order to reduce the computational complexity, we use the Lovejoy's [27][30] upper bound approximation. The basis of this approximation is: Randomly pick a number of points in information state simplex S, and approximate the estimation error with the piecewise linear interpolation compromising of tangents to $g^{\prime}(\pi) \pi$ at these points. From the previous chapter, we know that the tangent at point $\pi_{r}$ is the linear segment:

$$
g_{r}^{\prime}\left(\pi_{r}\right)=\left(1+\pi_{r}^{\prime} \pi_{r}\right)\left(\begin{array}{cc}
1 & 1
\end{array}\right)^{\prime}-2 \pi_{r}, r=1,2, \ldots, R
$$

In our simulation, we compared the result of $R=36$ points with that of $R=64$ points and the results are almost same. Therefore, we safely choose $R=36$ points for approximation. This comparison is shown in result 2 .

With the above setups, we use the POMDP program available from the website [24] to optimally solve the HMM sensor scheduling problem. The "Incremental Pruning" algorithm is used in our simulations. We consider the infinite horizon with discounted cost function $\beta=0.9$.

\subsubsection{Results}

\section{Result 1 - Approximate infinite horizon with horizon 200}

The infinite horizon cost function can be approximated by finite horizon cost function with large enough horizon $N$. In Figure 5.1, the cost over different horizons is shown. 


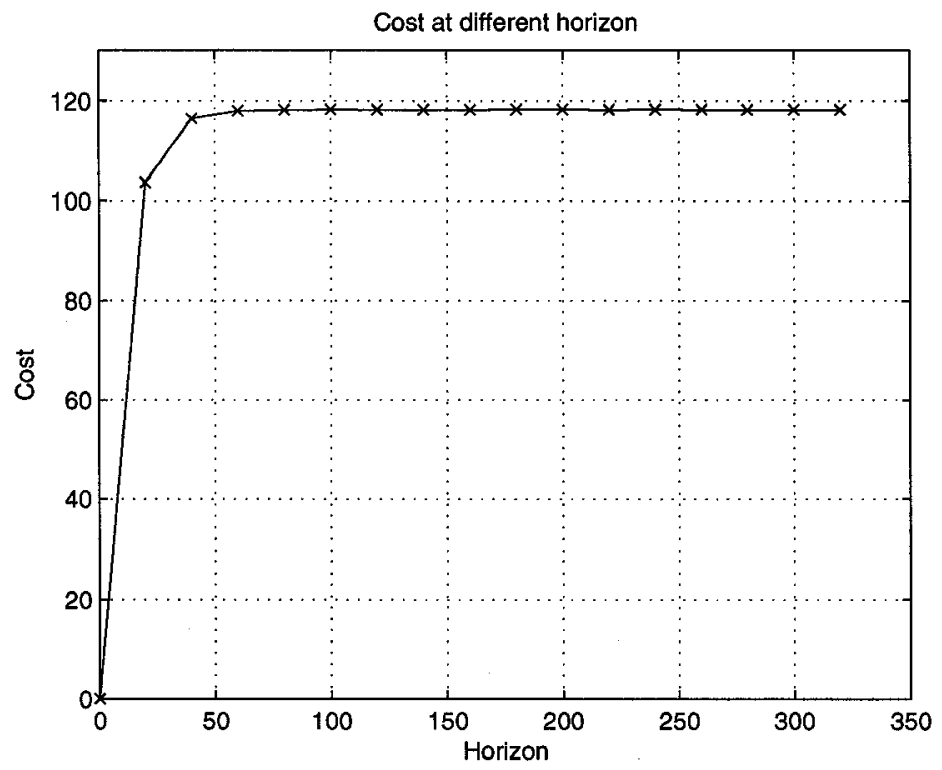

Fig. 5.1 Cost at different horizon.

We can see that the cost changes little after horizon $=100$. Therefore, it is reasonable to use horizon $=200$ to approximate our problem with infinite horizons.

\section{Result 2 - Choose $R=36$ for Lovejoy's approximation:}

Figure 5.2 and Figure 5.3 show the cost incurred versus $1-F A R$ (or $1-F R R$ ) if only the iris biosensor or prediction sensor is used, and also the cost of optimal sensor scheduling. We can see that the results of $R=64$ and $R=36$ are almost the same. Therefore, we safely choose $R=36$ for Lovejoy's approximation which can decrease the computation complexity.

\section{Result 3 - Optimal sensor scheduling always keeps the lowest cost:}

Figure 5.2 shows the costs incurred for the optimal sensor scheduling versus the probability $p=1-F R R=1-F A R$. Here we assume that $F R R=F A R$. The costs of using prediction sensor or using iris biosensor alone are also shown. It can be inferred 


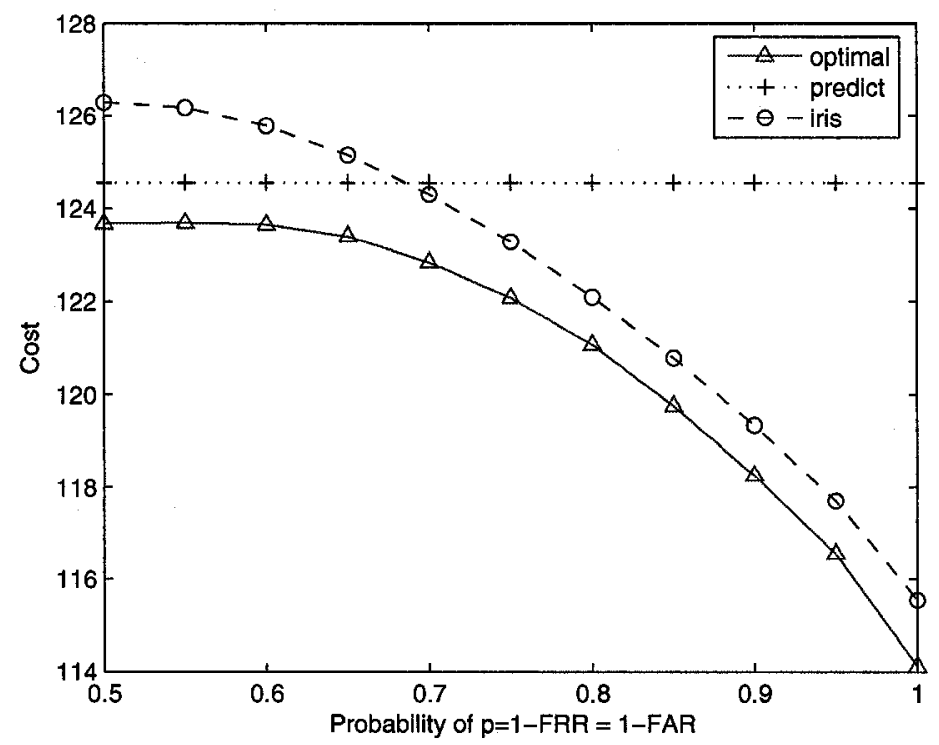

Fig. 5.2 Cost over infinite horizon with approximation points $\mathrm{R}=36$.

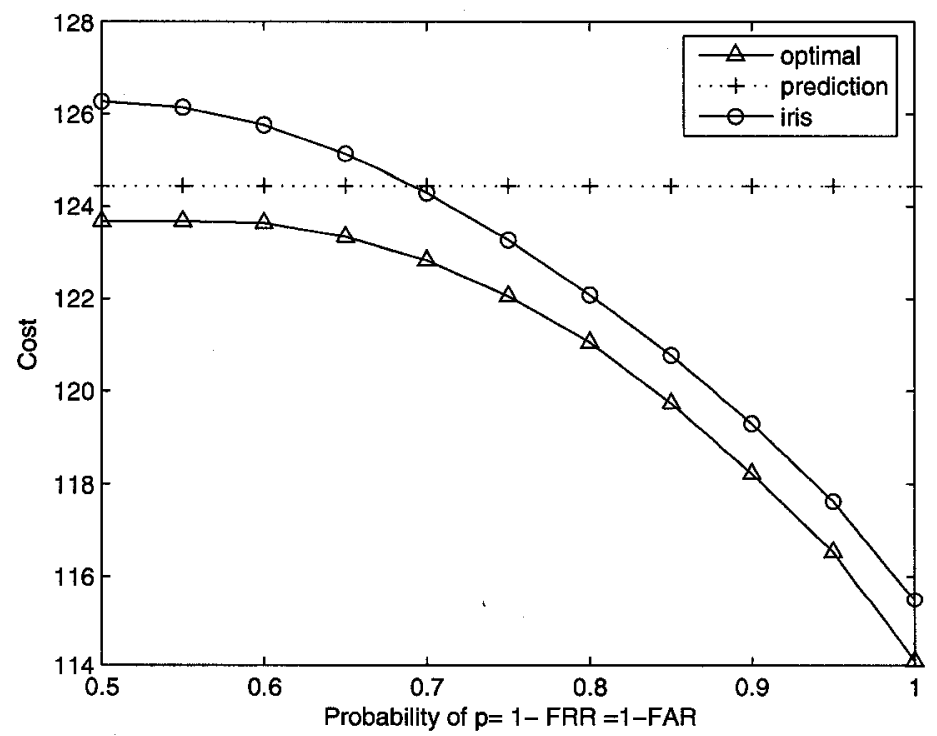

Fig. 5.3 Cost over infinite horizon with approximation points $\mathrm{R}=64$. 
that when the probability $p$ of iris biosensor is low (i.e., $0.5<p<0.67$ ), using prediction sensor is better than using iris biosensor. The reason is that the iris biosensor with low $p$ will incur higher estimation error. But as the probability $p$ increases, the cost of iris biosensor will decrease since the estimation error incurred will be reduced. Our optimal sensor schedule always has the lowest cost.

\section{Result 4-Optimal stationary policy over the information state:}

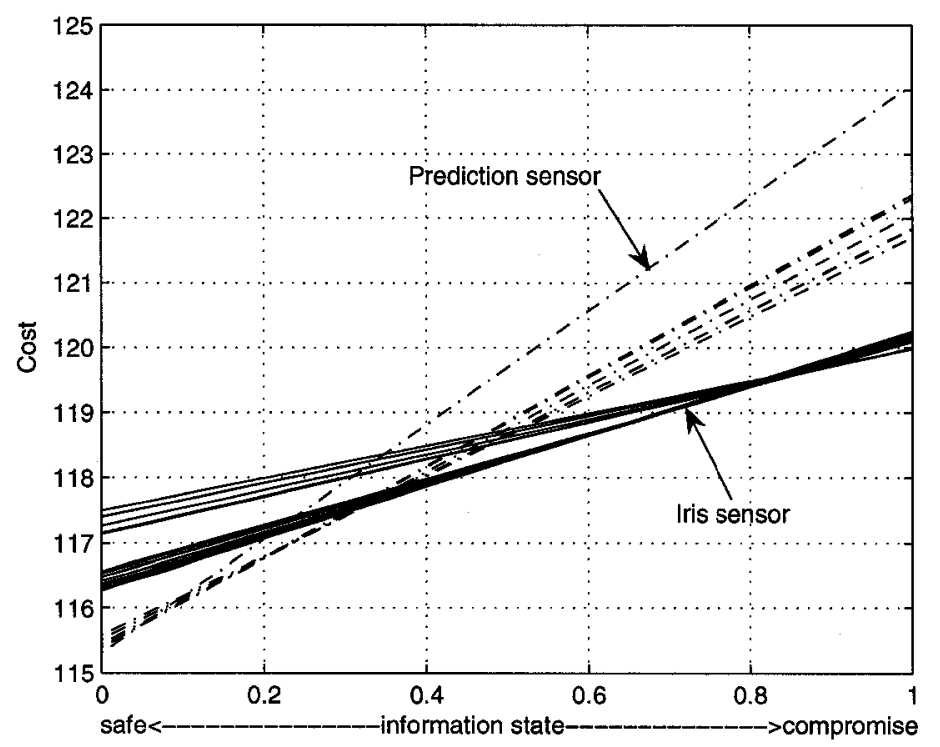

Fig. 5.4 Policy over information state with $\mathrm{FAR}=\mathrm{FRR}=0.1$.

Since the cost over the information space is piecewise and convex, the value function can be represented by a finite set of vectors such as:

$$
J^{k}(\pi)=\min _{i \in \Gamma^{k}}\left(\gamma_{i}^{k}\right)^{\prime} \pi \text { for all } \pi \in F
$$

where $\Gamma^{k}$ is a finite set of $S$-dimensional vectors $\left(\gamma_{i}^{k}\right)^{\prime}$. Figure 5.4 shows the vectors which represent the value function over the information space when the $F A R=0.1$. 
The value function is the lower surface of a finite number of linear segments. For system with two states, the linear segments is one-dimension lines, and for system with three or more states, the linear segments are hyper-planes through information space. Each hyper-plane or line can be represented with a vector of numbers using the coefficients of the equation of the hyper-plane or line. The value at any given information state is calculated by plugging the belief state into the hyper-plane or line equation. If we represent the hyper-plane or line with a vector with the equation coefficients, and the information state as a vector (the probability at each state) then the value of the information state is simply the dot product of the two vectors.

These lines in the Figure 5.4 completely specify the value function (over information space) that we desire. In this figure, each vector is a line connected with an action. Therefore, for each information state, we can acquire the optimal action once we compute which vector is used for representing the value function. In this figure, the solid line denotes using iris biosensor and the dashdot line denotes using the predictor sensor. We can easily see that for information space over [0.32 1], it is optimal to use iris biosensor, while for information space over [0 0.32$]$, using prediction sensor is better choice.

\section{Result 5 - Optimal stationary policy over the information state with dif- ferent FAR:}

Figure 5.5 shows the stationary scheduling policy on the information state simplex. For two-state HMM, the information simplex is a one-dimension line. The thickness of this line will serve only to help later clarification. The dark region denotes the information space $\pi$ for which it is optimal to use the iris biosensor, while the grey region denotes the prediction sensor will be optimally selected. In other words, no sensor will be used, and we only predict the device state with the HMM state predictor. 

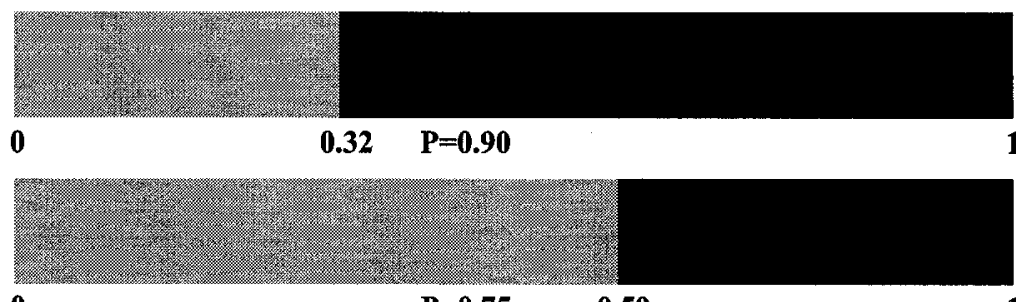

$\mathbf{0}$

$\mathbf{P}=\mathbf{0 . 7 5} \quad 0.59$ 1

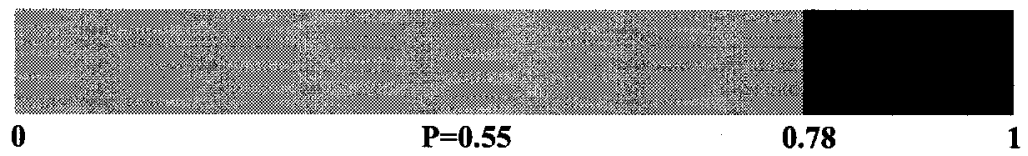

0 Safe $\leftarrow$

Information Space

$\rightarrow$ Compromised 1

Fig. 5.5 Sensor using over information space, $p=1-F A R=1-$ FRR.

The top line in Figure 5.5 shows that when iris biosensor genuine accept rate or genuine reject rate $p=0.90=1-F R R=1-F A R$, the iris biosensor is selected over more information space. It makes sense since higher accuracy sensor helps to distinguish the status of the device. With the increase of probability $p$, the numbers of using iris biosensor will grow accordingly. The reason is that the iris sensor is more accurate and the estimation error becomes lower than the case with $p=0.55$.

\section{Result 6 - Iris sensor using in different environments:}

Transition probability matrix $P$ defined in equation (5.1) denotes the safety status of the device. The larger the value of $P_{11}$, the safer the device. We simulated the cases with different transition probability and the results are shown in Figure 5.6. $\mathrm{Y}$ axis shows the average number of times that the iris biosensor is used over 30 horizons for five runs, and $\mathrm{X}$ axis shows the value of $P_{11}$. From this figure, we can see a trend that the number of times that the the iris biosensor is used decreases when $P_{11}$ becomes larger. It is reasonable since $P_{11}$ denotes the safety status of device. In safe environment, the frequency of using iris biosensor will be less than that in dangerous 


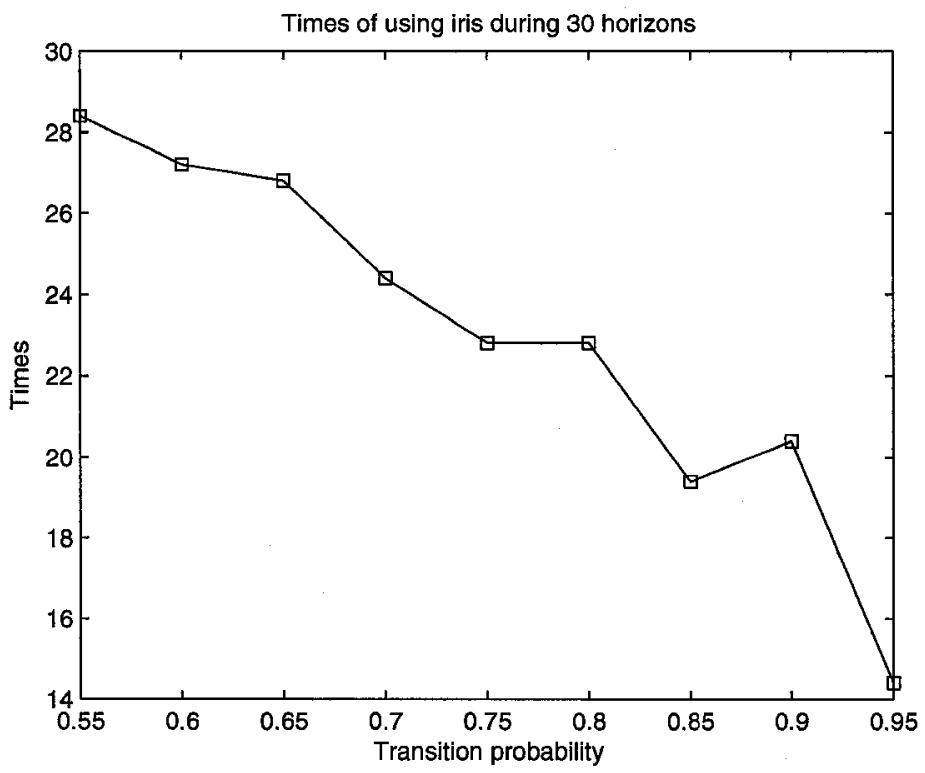

Fig. 5.6 Number of times using iris biosensor over 30 horizons with different transition probability.

environment.

\section{Result 7 - Estimation Error Constraints:}

We then consider quadratic constraints in estimation error, which are defined in equation (4.29). Since the observation matrix of the prediction sensor is:

$$
B\left(a^{k}=\text { prediction, } y^{k}=1\right)=\left(\begin{array}{ccc}
1 & 0 & 0 \\
0 & 1 & 0 \\
0 & 0 & 1
\end{array}\right)
$$

equation (4.29) can be rewritten as:

$$
u^{k+1} \text { (prediction) }\left(1-\pi^{\prime} P P^{\prime} \pi\right)<K_{\text {prediction }}
$$


In our simulations with constraints, $u^{k+1}$ (prediction) $=3, K_{\text {prediction }}=1.4$, and transition matrix $P$ is the same as equation (5.1). The observation matrix is:

$$
\hat{B}\left(a^{k}=\text { iris }\right)=\left(\begin{array}{ccc}
0.7 & 0.3 & 0 \\
0.3 & 0.7 & 0
\end{array}\right)
$$

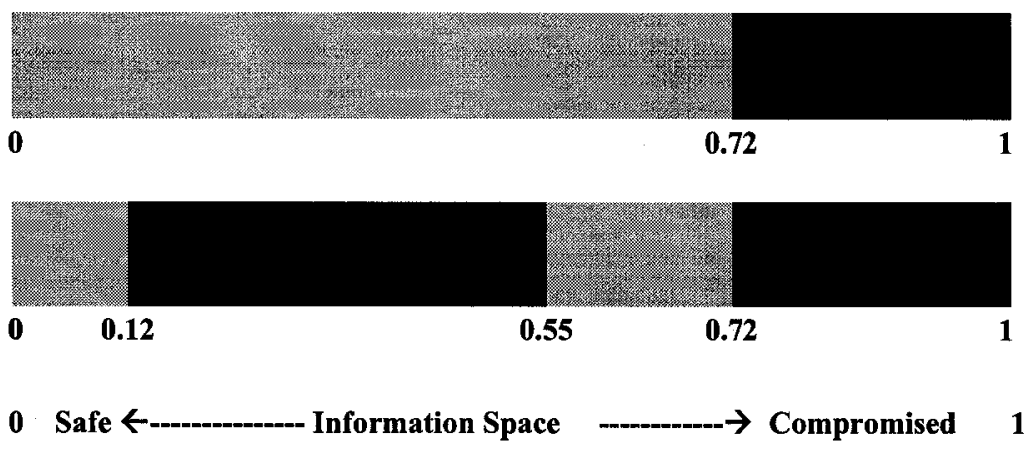

Fig. 5.7 Sensors usage over information space with and without constraints (top line - without constraints; bottom line - with constraints).

Figure 5.7 shows the information state simplex and the optimal stationary policy for the constraint and unconstraint cases. The dark region denotes the values of $\pi$ for which the iris biosensor is optimal, while the grey region denotes that the prediction sensor is optimal. The top line shows the sensor usage without estimation error constraints, while the bottom line shows the sensor usage with estimation error constraints. Compared with the top line, one more dark region over the information space is added to the bottom line. This region means that if the prediction sensor is selected at the next time instant, the estimation error incurred by this sensor will exceed the threshold. Therefore, the more accurate iris biosensor will be picked instead. 


\section{Result 8 - Resource constraints:}

We consider the resource constraints. Assume that the MANET is power-limited, and the iris biosensor can only be used at most 5 times. According to the definition in equation (4.30), $N=5$. There are 12 information states for this Markov process. 0 means that the device stays in the safe state and the iris biosensor has never been used. $1,2,3,4,5$ mean that the device stays in the safe state and the iris biosensor has been used once, twice, 3 times, 4 times, and 5 times, respectively. 6 means that the device stays in the compromised state and the iris biosensor has never been used. $7,8,9,10,11$ mean that the device stays in the compromised state and the iris biosensor has been used once, twice, 3 times, 4 times, and 5 times, respectively.

For the transition matrix $\bar{P}$, if the iris biosensor is selected,

$$
P=P \otimes Q=\left(\begin{array}{cccccccccccc}
0 & 0.7 & 0 & 0 & 0 & 0 & 0 & 0.3 & 0 & 0 & 0 & 0 \\
0 & 0 & 0.7 & 0 & 0 & 0 & 0 & 0 & 0.3 & 0 & 0 & 0 \\
0 & 0 & 0 & 0.7 & 0 & 0 & 0 & 0 & 0 & 0.3 & 0 & 0 \\
0 & 0 & 0 & 0 & 0.7 & 0 & 0 & 0 & 0 & 0 & 0.3 & 0 \\
0 & 0 & 0 & 0 & 0 & 0.7 & 0 & 0 & 0 & 0 & 0 & 0.3 \\
0 & 0 & 0 & 0 & 0 & 0.7 & 0 & 0 & 0 & 0 & 0 & 0.3 \\
0 & 0.1 & 0 & 0 & 0 & 0 & 0 & 0.9 & 0 & 0 & 0 & 0 \\
0 & 0 & 0.1 & 0 & 0 & 0 & 0 & 0 & 0.9 & 0 & 0 & 0 \\
0 & 0 & 0 & 0.1 & 0 & 0 & 0 & 0 & 0 & 0.9 & 0 & 0 \\
0 & 0 & 0 & 0 & 0.1 & 0 & 0 & 0 & 0 & 0 & 0.9 & 0 \\
0 & 0 & 0 & 0 & 0 & 0.1 & 0 & 0 & 0 & 0 & 0 & 0.9 \\
0 & 0 & 0 & 0 & 0 & 0.1 & 0 & 0 & 0 & 0 & 0 & 0.9
\end{array}\right),
$$


if the iris biosensor is not selected,

$$
\bar{P}=P \otimes I_{N+1}=\left(\begin{array}{cccccccccccc}
0.7 & 0 & 0 & 0 & 0 & 0 & 0.3 & 0 & 0 & 0 & 0 & 0 \\
0 & 0.7 & 0 & 0 & 0 & 0 & 0 & 0.3 & 0 & 0 & 0 & 0 \\
0 & 0 & 0.7 & 0 & 0 & 0 & 0 & 0 & 0.3 & 0 & 0 & 0 \\
0 & 0 & 0 & 0.7 & 0 & 0 & 0 & 0 & 0 & 0.3 & 0 & 0 \\
0 & 0 & 0 & 0 & 0.7 & 0 & 0 & 0 & 0 & 0 & 0.3 & 0 \\
0 & 0 & 0 & 0 & 0 & 0.7 & 0 & 0 & 0 & 0 & 0 & 0.3 \\
0.1 & 0 & 0 & 0 & 0 & 0 & 0.9 & 0 & 0 & 0 & 0 & 0 \\
0 & 0.1 & 0 & 0 & 0 & 0 & 0 & 0.9 & 0 & 0 & 0 & 0 \\
0 & 0 & 0.1 & 0 & 0 & 0 & 0 & 0 & 0.9 & 0 & 0 & 0 \\
0 & 0 & 0 & 0.1 & 0 & 0 & 0 & 0 & 0 & 0.9 & 0 & 0 \\
0 & 0 & 0 & 0 & 0.1 & 0 & 0 & 0 & 0 & 0 & 0.9 & 0 \\
0 & 0 & 0 & 0 & 0 & 0.1 & 0 & 0 & 0 & 0 & 0 & 0.9
\end{array}\right) .
$$


For the observation matrix $B$,

$$
\bar{B}(\mathrm{safe})=\left(\begin{array}{cccccccccccc}
0.9 & 0 & 0 & 0 & 0 & 0 & 0 & 0 & 0 & 0 & 0 & 0 \\
0 & 0.9 & 0 & 0 & 0 & 0 & 0 & 0 & 0 & 0 & 0 & 0 \\
0 & 0 & 0.9 & 0 & 0 & 0 & 0 & 0 & 0 & 0 & 0 & 0 \\
0 & 0 & 0 & 0.9 & 0 & 0 & 0 & 0 & 0 & 0 & 0 & 0 \\
0 & 0 & 0 & 0 & 0.9 & 0 & 0 & 0 & 0 & 0 & 0 & 0 \\
0 & 0 & 0 & 0 & 0 & 0.9 & 0 & 0 & 0 & 0 & 0 & 0 \\
0 & 0 & 0 & 0 & 0 & 0 & 0.1 & 0 & 0 & 0 & 0 & 0 \\
0 & 0 & 0 & 0 & 0 & 0 & 0 & 0.1 & 0 & 0 & 0 & 0 \\
0 & 0 & 0 & 0 & 0 & 0 & 0 & 0 & 0.1 & 0 & 0 & 0 \\
0 & 0 & 0 & 0 & 0 & 0 & 0 & 0 & 0 & 0.1 & 0 & 0 \\
0 & 0 & 0 & 0 & 0 & 0 & 0 & 0 & 0 & 0 & 0.1 & 0 \\
0 & 0 & 0 & 0 & 0 & 0 & 0 & 0 & 0 & 0 & 0 & 0.1
\end{array}\right),
$$




$$
\bar{B} \text { (compromised) }=\left(\begin{array}{cccccccccccc}
0.1 & 0 & 0 & 0 & 0 & 0 & 0 & 0 & 0 & 0 & 0 & 0 \\
0 & 0.1 & 0 & 0 & 0 & 0 & 0 & 0 & 0 & 0 & 0 & 0 \\
0 & 0 & 0.1 & 0 & 0 & 0 & 0 & 0 & 0 & 0 & 0 & 0 \\
0 & 0 & 0 & 0.1 & 0 & 0 & 0 & 0 & 0 & 0 & 0 & 0 \\
0 & 0 & 0 & 0 & 0.1 & 0 & 0 & 0 & 0 & 0 & 0 & 0 \\
0 & 0 & 0 & 0 & 0 & 0.1 & 0 & 0 & 0 & 0 & 0 & 0 \\
0 & 0 & 0 & 0 & 0 & 0 & 0.9 & 0 & 0 & 0 & 0 & 0 \\
0 & 0 & 0 & 0 & 0 & 0 & 0 & 0.9 & 0 & 0 & 0 & 0 \\
0 & 0 & 0 & 0 & 0 & 0 & 0 & 0 & 0.9 & 0 & 0 & 0 \\
0 & 0 & 0 & 0 & 0 & 0 & 0 & 0 & 0 & 0.9 & 0 & 0 \\
0 & 0 & 0 & 0 & 0 & 0 & 0 & 0 & 0 & 0 & 0.9 & 0 \\
0 & 0 & 0 & 0 & 0 & 0 & 0 & 0 & 0 & 0 & 0 & 0.9
\end{array}\right) .
$$

Figure 5.8 and Figure 5.9 show the simulation results of using the iris biosensor with resource constraints and without resource constraints, respectively. $p$ in the figures stands for the genuine acceptance rate and genuine rejection rate of the iris biosensor. An arrow means that the iris biosensor is used at that time instant. From these two figures, we can see that the iris biosensor will be used for much more times if there is no resource constraints. On the other hand, if there are resource constraints in the system (the iris biosensor can be used for at most 5 times), our scheme can guarantee resource constraints. We also observe that the iris biosensor is used for more times when $p=0.9$ compared to the case when $p=0.8$. 

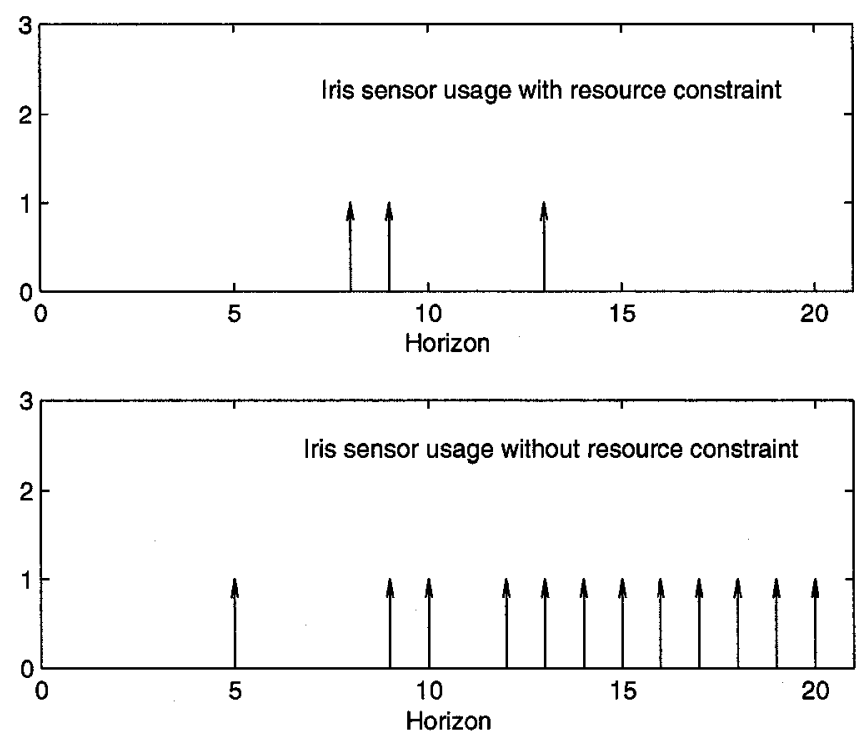

Fig. 5.8 Times of using iris biosensor when $\mathrm{p}=1$ - FRR $=1$-FAR $=0.8$.
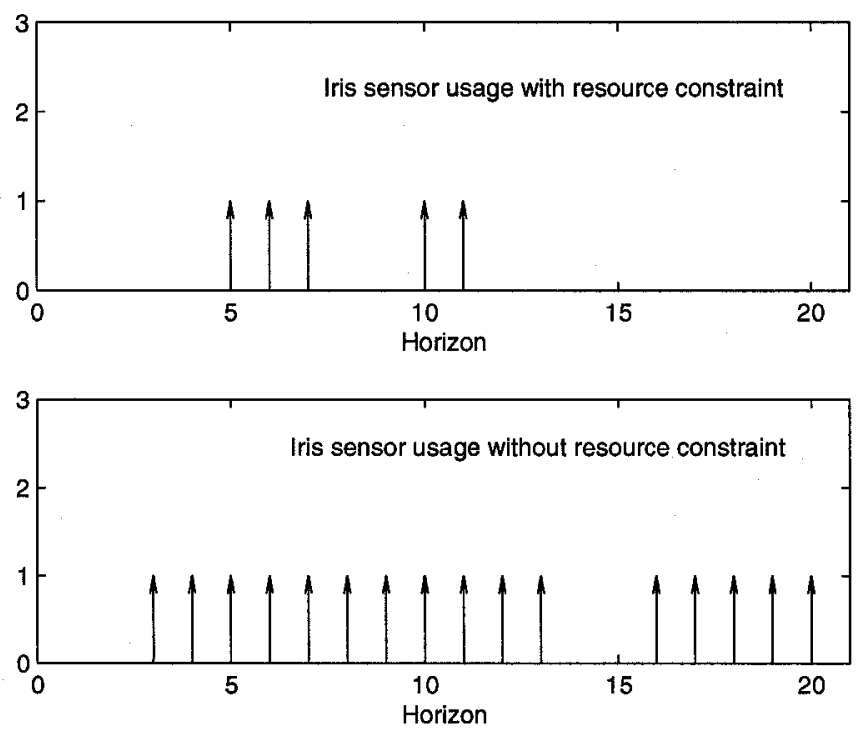

Fig. 5.9 Times of using iris biosensor when $\mathrm{p}=1-\mathrm{FRR}=1-\mathrm{FAR}=0.9$. 


\subsection{Scenario 2 - Multi-modal Biometric Continuous}

\section{Authentication}

\subsubsection{Parameters}

In this scenario, one more sensor will be added to simulate the multimodal biosensor based authentication.

State space: We take the same definition as Scenario 1. The state is the status of the device: safe or compromised. The transition probability matrix of $X^{k}$ is

$$
P=\left(\begin{array}{cc}
0.85 & 0.15 \\
0.1 & 0.9
\end{array}\right)
$$

Sensors: Three sensors will be used: one iris biosensor, one fingerprint biosensor, and one prediction sensor. The iris biosensor is more accurate than the fingerprint biosensor but the cost is higher than that of fingerprint biosensor.

Observation symbols: Three observation symbols will be used in this scenario, which are the same as those in scenario 1: acceptance, rejection, and nothing. The observation matrix is defined as:

$$
\begin{aligned}
& \hat{B}\left(a^{k}=\text { iris }\right)=\left(\begin{array}{lll}
0.95 & 0.05 & 0 \\
0.05 & 0.95 & 0
\end{array}\right), \\
& \hat{B}\left(a^{k}=\text { fingerprint }\right)=\left(\begin{array}{lll}
0.8 & 0.2 & 0 \\
0.2 & 0.8 & 0
\end{array}\right),
\end{aligned}
$$




$$
\hat{B}\left(a^{k}=\text { prediction }\right)=\left(\begin{array}{lll}
0 & 0 & 1 \\
0 & 0 & 1
\end{array}\right) \text {. }
$$

Cost function: There are two components in the cost function. The explanation of these parameters is the same as that in scenario 1 :

- Sensor costs:

$$
\begin{aligned}
& c\left(X^{k}=e_{i}, a^{k+1}=\text { iris }\right)=\rho^{\text {iris }}+r^{\text {iris }}, \\
& c\left(X^{k}=e_{i}, a^{k+1}=\text { prediction }\right)=\rho^{\text {prediction }}+r^{\text {prediction }}, \\
& c\left(X^{k}=e_{i}, a^{k+1}=\text { finger }\right)=\rho^{\text {finger }}+r^{\text {finger }},
\end{aligned}
$$

where $\rho^{\text {iris }}=11.5>\rho^{\text {finger }}=7>\rho^{\text {prediction }}=4.5$ means that the sensor cost of using iris is higher than using fingerprint, and the cost of using fingerprint is higher than that of using prediction sensor. We set the estimation error cost as:

$$
\begin{aligned}
& r^{\text {iris }}\left(X^{k}=0\right)=0.5, r^{\text {iris }}\left(X^{k}=1\right)=0.7 \\
& r^{\text {finger }}\left(X^{k}=0\right)=1.0, r^{\text {finger }}\left(X^{k}=1\right)=4.5, \\
& r^{\text {prediction }}\left(X^{k}=0\right)=1.5, r^{\text {prediction }}\left(X^{k}=1\right)=9 .
\end{aligned}
$$

Under the most possibly compromised situations, using the sensor with lowest accuracy will bring the highest information leaking.

- Estimation error: We use the same method defined in equation (5.4) to 
approximate the quadratic estimation error. Here we set $u^{k}=3$ and still use $R=36$ points for approximation.

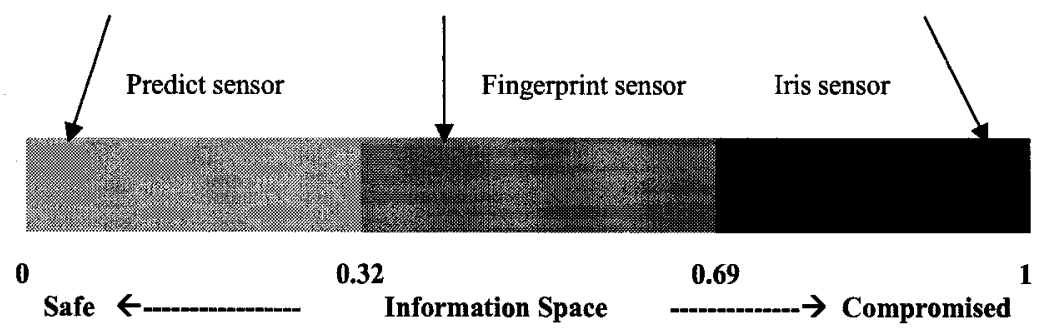

Fig. 5.10 Sensors usage over information space.

\subsubsection{Results}

Figure 5.10 shows the simulation results. The line in the figure shows the two-state information simplex. Dark region - the right hand part indicates the information $\pi$ for which the iris biosensor is optimal; Light grey region - the left hand part shows the information state $\pi$ for which the prediction sensor is optimal; In the middle of the information space, it is optimal to use the fingerprint biosensor. That makes the intuitive sense that right part indicates that the device is mostly compromised and the most accurate sensor will be helpful to distinguish the compromised device. While the left part of information space indicates that the device is in safe mode, so we do not need authenticate this device in short time. The middle part means that we are not certain about the status of the device. With the sensor usage costs, using the fingerprint biosensor will be the best choice.

\subsection{Scenario 3 - Multi-server Scheduling}

In this subsection, we present the numerical example for multi-server scheduling. 


\subsubsection{Parameters}

In this example, we assume that there are two types of servers that can serve as authentication server. For example, these two types of servers are controlled by group leaders with different ranks. The number of servers belonging to the same type depends on the MANET scale.

We consider that each server has two state information emission levels: $s^{k} \in$ $\{l o w$, high $\}$. Therefore, each server can be modeled as a two-state Markov chain, i.e., $N_{l}=2$. In all cases, each server can access the IDS at any time to get the threat level posted to each server.

\section{Parameters of Type 1 Server:}

$$
\begin{aligned}
& P^{(\text {Type1 })}=\left(\begin{array}{ll}
0.8 & 0.2 \\
0.1 & 0.9
\end{array}\right), \\
& B^{(\text {Type1 })}=\left(\begin{array}{ll}
0.8 & 0.2 \\
0.3 & 0.7
\end{array}\right), \\
& R^{(\text {Type1 })}=\left(\begin{array}{l}
9 \\
4
\end{array}\right) .
\end{aligned}
$$

The probability 0.8 in transition probability matrix means that the information emission level of type 1 server will keep low with $80 \%$ probability after one time horizon when it acts as the authentication server. The probability 0.1 means that if the type 1 server stays in high information emission level, the countermeasures are effective in mitigating the threat level to low with probability 0.1 . The observation matrix denotes the precision of IDS, which is used to evaluate the threat level posted to each server. Here $B_{12}=F P R$ and $B_{21}=F N R$ of 
IDS. The observed threat levels are a probabilistic function of the unobserved finite information emission levels. We set the cost $C(s(1)=l o w)=-9$, and $C(s(1)=h i g h)=-4$. For the reward value, we take the negative cost values as reward values. Therefore, we minimize the cost by maximizing the reward. When a server stays in high information emission level, the cost of being authentication server will be higher than that stays in low information emission level. This is reasonable because one more authentication activity could make the authentication server easily detected by the enemy when the server already stays in high threat level. We take negative cost as reward value, which is described before.

\section{Parameters of Type 2 Server:}

$$
\begin{aligned}
& P^{(\text {Type2 })}=\left(\begin{array}{cc}
0.85 & 0.15 \\
0.2 & 0.8
\end{array}\right), \\
& B^{(\text {Type2) }}=\left(\begin{array}{cc}
0.85 & 0.15 \\
0.2 & 0.8
\end{array}\right), \\
& R^{(\text {Type2 })}=\left(\begin{array}{c}
7.5 \\
5
\end{array}\right) .
\end{aligned}
$$

The definitions of parameters for server 2 are the same as those in server 1 . We choose the discount factor $\beta=0.8$ for both server 1 and server 2 .

\subsubsection{Results}

1. Off-line computation of Gittins index: The Gittins indices of the 2 types of servers are computed as follows: 


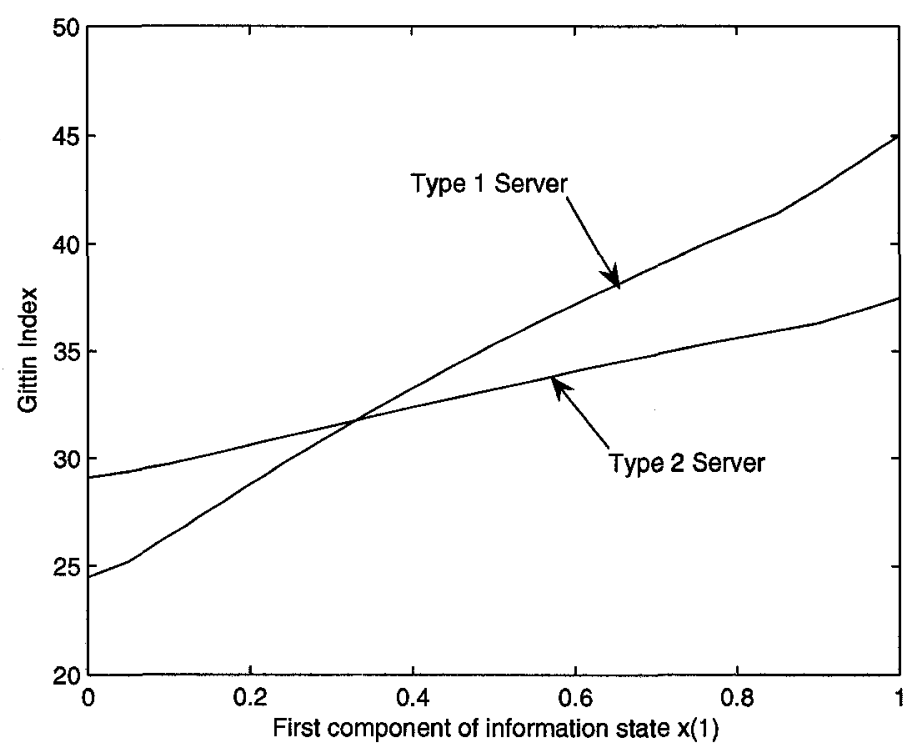

Fig. 5.11 Gittins indices for two types of servers.

First we use the POMDP program from the website [24] to compute the set of vectors $\Lambda^{N}(1), \Lambda^{N}(2)$ for horizon 20 . We choose the "Incremental Pruning" algorithm to solve the POMDP problem. The numerical resolution of $\epsilon=10^{-3}$ is selected. The Gittins indices for two types of servers $\gamma^{N}(1), \gamma^{N}(2)$ are plotted in Figure 5.11. Because $N_{l}=2$, and $x(1)+x(2)=1$, we plot $\gamma^{N}(l, x)$ versus $x(1)$.

2. Real-time Emission Level: After computing $\Lambda^{N}($.$) as described above, for$ all the servers, HMM filters can be implemented as outlined in section 4.2. 


\subsection{Summary}

In Subsections 5.1 and 5.2, we presented the numerical examples of user-to-device authentication in proposed sensor scheduling algorithms. The results show that the examples using optimal algorithms do better than using any biosensor alone. With the optimal biosensor scheduling algorithms, the continuous authentication process can select the biosensor dynamically at each time instant according to the system status. System security requirement constraints and resource constraints can be guaranteed. In Subsection 5.3, the example of computing the Gittins indices was illustrated. With our algorithms, the servers are indexable, and the network is decentralized and scalable. With minimum communication, the servers can dynamically regulate their information emission and hence decrease the total network threat level.

In the numerical examples and simulations, we used the assumption parameters we needed, such as transition probability matrix, observation probability matrix, and etc. The simulation results would be more realistic if these parameters can be obtained from actual experiments. 


\section{Chapter 6}

\section{Conclusions and Future Work}

In MANETs, there are two classes of user authentication: user-to-device and user-tonetwork authentication. For user-to-device authentication, one-time authentication is inadequate, especially in some tactical environments. Extra requirements such as little resource should be used for authentication schemes exist in MANETs. Multimodal biometrics provides the possibility to meet all the requirements of authentication in MANETs. In this thesis, we have formulated the user-to-device continuous authentication process as a POMDP problem: the device state cannot be observed directly and hence the decision will be made based on the result of using biosensors. Each biosensor is connected with some energy consumed and estimation errors. By using the POMDP algorithms, continuous authentication turns into an optimal biosensor scheduling problem. A tradeoff between biosensor cost and estimation error can be balanced by POMDP algorithms. Our examples using optimal algorithms do better than using any biosensor alone. With our optimal biosensor scheduling algorithms, the continuous authentication process can select the biosensor dynamically at each time instant according to the system status.

For user-to-network authentication in MANETs, decentralized authentication strate- 
gies are desirable since there is no centralized authentication server available. We presented decentralized authentication algorithms for reducing the detectability of authentication servers to the enemy. In our decentralized authentication scheme, the users' biometric templates are stored in several distributed servers, and each server is formulated as a POMDP model. All the servers are indexable with a Gittins index, meaning that at each time instant it is optimal to select the server with the highest Gittins index as the authentication server . Given this multi-armed bandit structure and indexable nature, our scheme is fully decentralized and easily scalable since the Gittins index is computed for each server separately of every other server. By taking into account the threat information acquired from the IDS, our scheme can dynamically select which server will act as the authentication server to minimize the threat level posted to the whole network.

In the proposed user-to-device scheme, the length of time interval can be changed according to the environment and security requirements of MANETs. Basically, for MANETs which require high security or stay in hostile environments, the time interval can be set shorter than those need low security or stay in safe environments. Moreover, the time interval should be longer than authentication time. It is important that the transition probability matrix needs to be changed accordingly if time interval length is changed. In the future, In the future, different time intervals and transition probabilities for various scenarios can be investigated.

The future work will also consider how to combine the user-to-device algorithms with some biosensor authentication schemes. In [3], the authors presented some new metrics to evaluate the performance of continuous authentication by incorporating the time factor. Therefore, in the future, we will investigate how to use these metrics to measure the performance of our algorithms. Another future research area is to investigate the possibility to apply our decentralized user-to-network algorithms to 
heterogeneous networks. Military networks usually compromise different types of devices and sophisticated platforms. The Gittins index approach can provides good solutions to the security problems in heterogeneous networks. 


\section{References}

[1] A. Weimerskirch and G. Thonet, "A Distributed Light-Weight Authentication Model for Ad-hoc Networks," Lecture Notes in Computer Science, vol. 2288, pp. 341-354, ISBN: 3-540-43319-8, 2001.

[2] Q. Xiao, "A Biometric Authentication Approach for High Security Ad-hoc Networks," in Proc. IEEE Info. Assurance Workshop, West Point, New York, June 2004.

[3] T. Sim, S. Zhang, R. Janakriaman and S. Kumar, "Continuous Verification Using Multimodal Biometrics," IEEE Trans. Patten Analysis and Machine Intelligence, vol. 29, no. 4, pp. 687-700, Apr. 2007.

[4] Y. Zhang, W. Lee, and Y. Huang, "Intrusion Detection Techniques for Mobile Wireless Networks," in ACM J. Wireless Net, vol. 9, no. 5, pp. 45-56, Sep. 2003.

[5] S. Gokhale and P. Dasgupta, "Distributed Authentication for Peer-to-Peer Networks," Proc. Applications and the Internet Workshops, pp. 347-353, ISBN: 07695-1873-7, Jan. 2003.

[6] C. Siva Ram Murthy and B. S. Manoj, Ad Hoc Wireless Networks: Architectures and Protocols. Prentice Hall, Upper Saddle River, NJ., 2004. 
[7] H. Yang, H. Luo, F. Ye, et al., "Security in Mobile Ad Hoc Networks: Challenges and Solutions," IEEE Wireless Communications, vol. 11, issue 1, pp. 38-47, Feb. 2004 .

[8] M. Guizani, Wireless Communications Systems and Networks. Kluwer Academic/Plenum Publishers, NY., 2004.

[9] J. Koreman, A. C. Morris, D. Wu, S. A. Jassim, et al, "Multi-modal Biometrics Authentication on the Securephone PDA," in Proc. Second Workshop on Multimodal User Authentication, Toulouse, France, May 2006.

[10] A. J. Klosterman and G. R. Ganger, "Secure Continuous Biometric-Enhanced Authentication," in CMU SCS Technical Report CMU-CS-00-134, May 2000.

[11] Document of BioID Company, [Online]. Available: http://www.bioid.com/sdk/docs/.

[12] A. Ross and A. K. Jain, "Multimodal Biometrics: An Overview," in Proc. of 12th European Signal Proc. Conf., Vienna, Austria, 2004.

[13] A. Ross and A. K. Jain, "Information Fusion in Biometrics," Pattern Recognition Letters, vol. 24, pp. 2115-2225, Sep. 2003.

[14] S. K. Das, A. Agah and K. Basu, "Security in Wireless Mobile and Sensor Networks," Wireless Communications Systems and Networks, pp. 531-557, ISBN: 0306-48190-1, 2004.

[15] A. Mishra, K. Nadkarni, and A. Patcha, "Intrusion Detection in Wireless Ad Hoc Networks," in IEEE Wireless Communications, vol. 11, no. 1, pp. 48-60, Feb. 2004. 
[16] M. Chappel, "Evaluating and Tuning an Intrusion-detection System," [Online]. Available:

http://searchsecurity.techtarget.com/tip/1,289483, sid14_ gci918619, 00.html?track=IDSLG.

[17] J. Muncaster and M. Turk, "Continuous Multimodal Authentication Using Dynamic Bayesian Networks," in Proc. Second Workshop on Multimodal User Authentication, Toulouse, France, May 2006.

[18] A. Altinok and M. Turk, "Temporal Integration for Continuous Multimodal Biometrics," Proc. Workshop Multimodal User Authentication, pp.131-137, Dec. 2003.

[19] R. D. Smallwood and E. J. Sondik, "Optimal Control of Partially Obervable Markov Processes over A Finite Horizon," in Oper. Res., vol. 21, no. 5, pp. 1071$1088,1973$.

[20] A. R. Cassandra, "Exact and Approximate Algorithms for Partially Observed Markov Decision Process," Ph. D. Dissertation, Department of Computer Science, Brown University, Province, RI, 1998.

[21] E. J. Sondik, "The Optimal Control of Partially Observable Markov Processes," PhD thesis, Stanford University, Stanford, Carlifornia, 1971.

[22] H. Cheng, "Algorithms for Partially Observed Markov Decision Processes," PhD thesis, University of British Columbia, British Columbia, Canada, 1998.

[23] N. L. Zhang and W. Liu, "Planning in stochastic domains: Problem characteristics and approximation," Technical Report HKUSTCS96-31, Department of Computer Science, Hong Kong University of Science and Technology, 1996. 
[24] A. R. Cassandra, "Tony's POMDP Webpage," [Online]. Available: http://www.cs.brown.edu/research/ai/pomdp/index.html.

[25] H. Tang, M. Salmanian, Q. Xiao, "Biometric-based User Authentication for Tactical Mobile Ad-Hoc Networks," Technical Note, 2007-100, Defense R\&D Canada, May 2007.

[26] V. Krishnamurthy, "Algorithms for Optimal Scheduling and Management of Hidden Markov Model Sensors," IEEE Trans. Signal Processing, vol. 50, no. 6, pp. 1382-1397, June 2002.

[27] W. S. Lovejoy, "Computationally Feasible Bounds for Partially Observed Markov Decision Processes," Oper. Res., vol. 39, no. 1, pp. 162-175, 1991.

[28] V. Krishnamurthy, "Emission Management for Low Probability Intercept Sensors in Network Centric Warfare," IEEE Trans. on Aerospace and Electronic Systems, vol. 41, no. 1, pp. 133-152, Jan. 2005.

[29] V. Krishnamurthy, "A Value Iteration Algorithm for Partially Observed Markov Decision Process Multi-armed Bandits," Mathematics of Operations Research, pp. 133-152, May 2005.

[30] W. S. Lovejoy, "A survey of algorithmic methods for partially observed Markov decision processes," Annals of Operations Research, vol. 28, pp. 47-66, 1991. 


\section{Appendix A: MATLAB Programs}

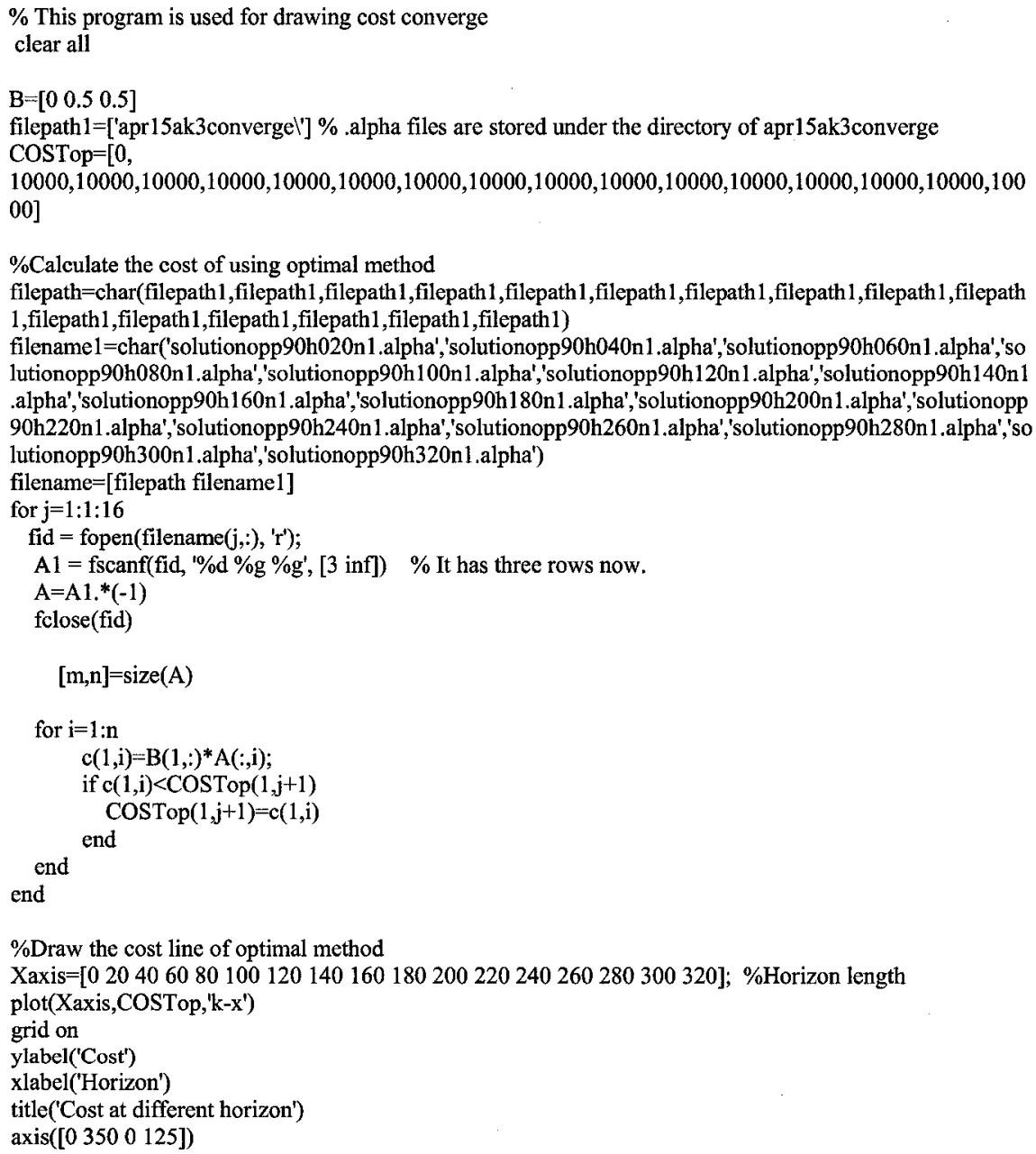

Fig. A-1 Matlab file for computing result 1 


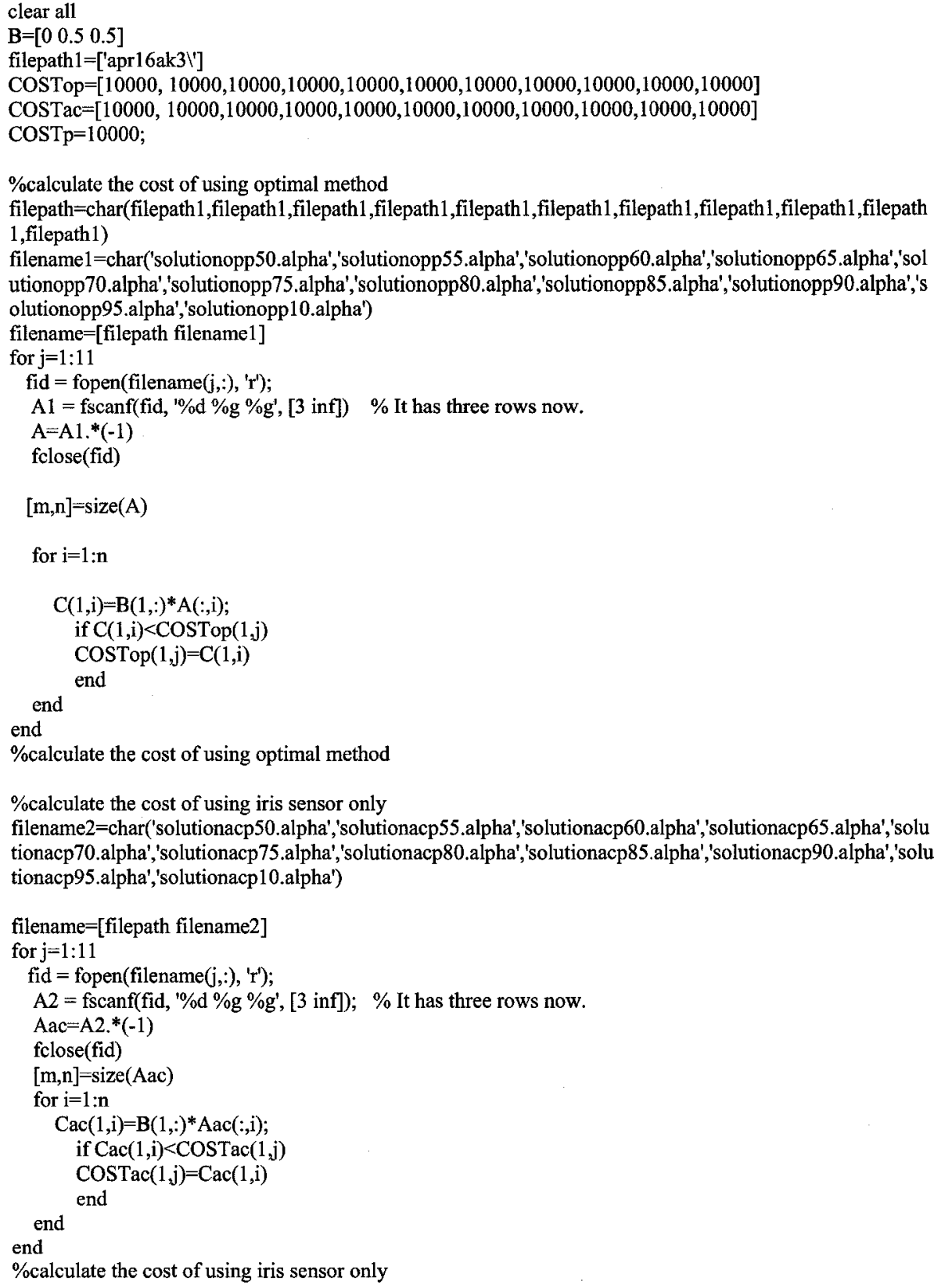

Fig. A-2 Matlab file for computing result 2 page 1 


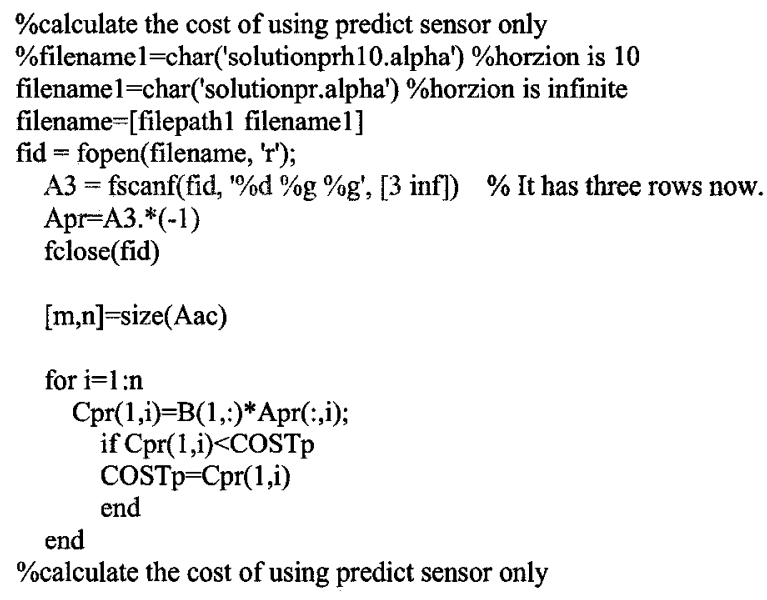

Xaxis=[ 0.50 .550 .60 .650 .700 .750 .80 .850 .90 .951 .0$]$; \%detection probility plot(Xaxis,COSTop,'r-x') \%draw the cost line of optimal method hold on

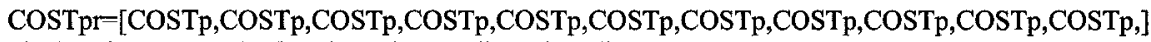
plot(Xaxis,COSTpr,'k:+') \%draw the cost line of predict sensor

hold on

plot(Xaxis,COSTac,'b--o') \%draw the cost line of iris sensor

hold off

$\mathrm{h}=$ legend('optimal','predict','iris',1);

set(h,'Interpreter','none')

Xlabel('Probability $\mathbf{P}=1-\mathrm{FRR}=1-\mathrm{FAP}$ ');

ylabel('Cost')

Fig. A-3 Matlab file for computing result 2 page 2 


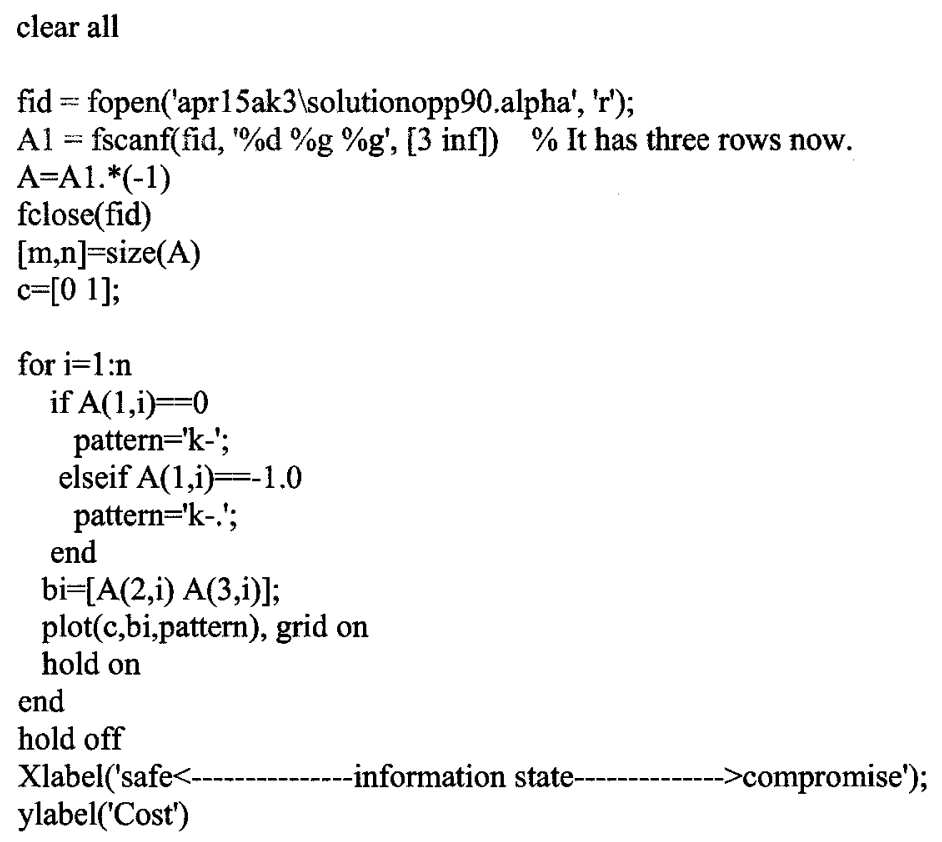

Fig. A-4 Matlab file for computing result 3 


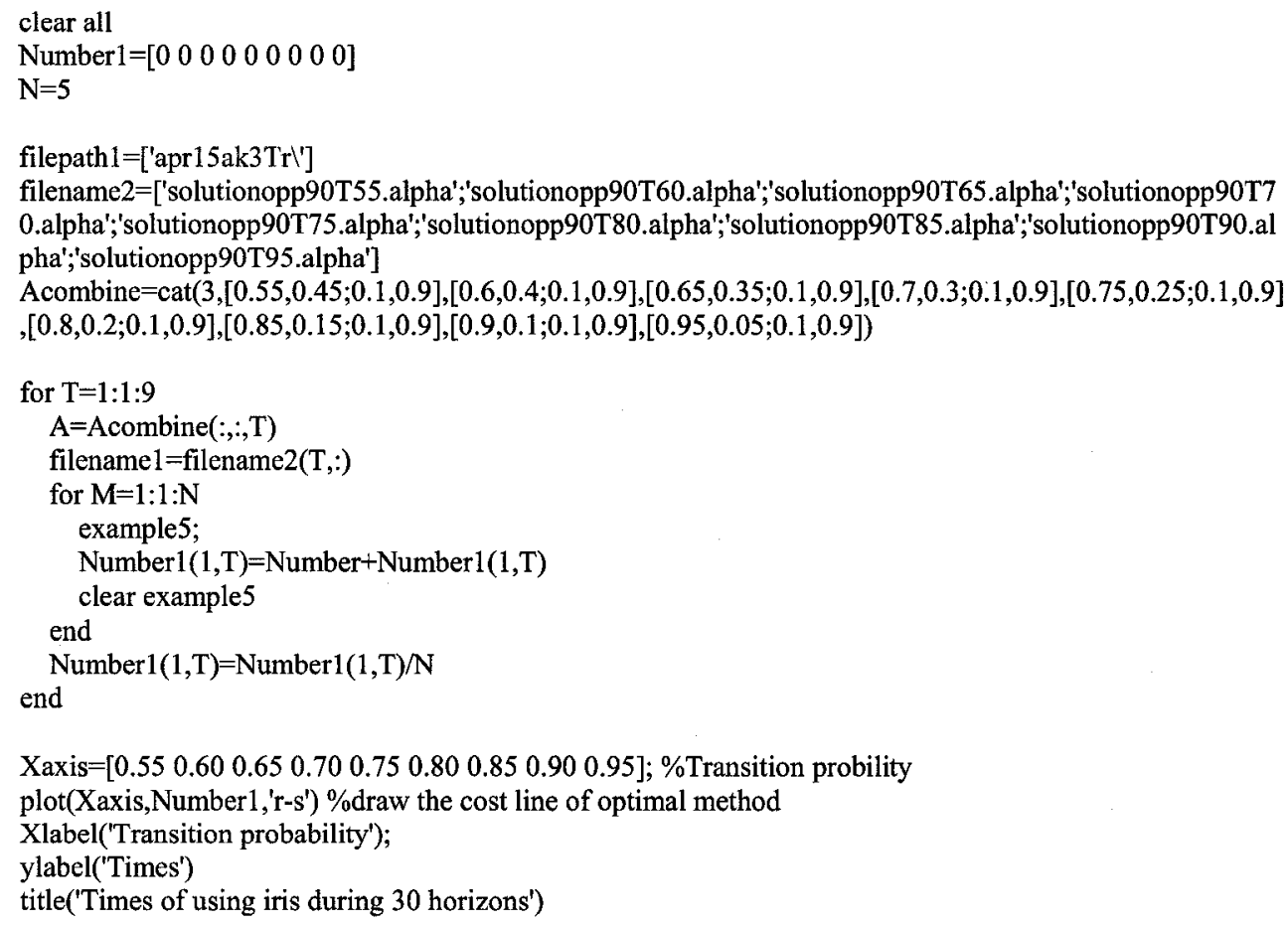

Fig. A-5 Matlab file for computing result 4 page 1 


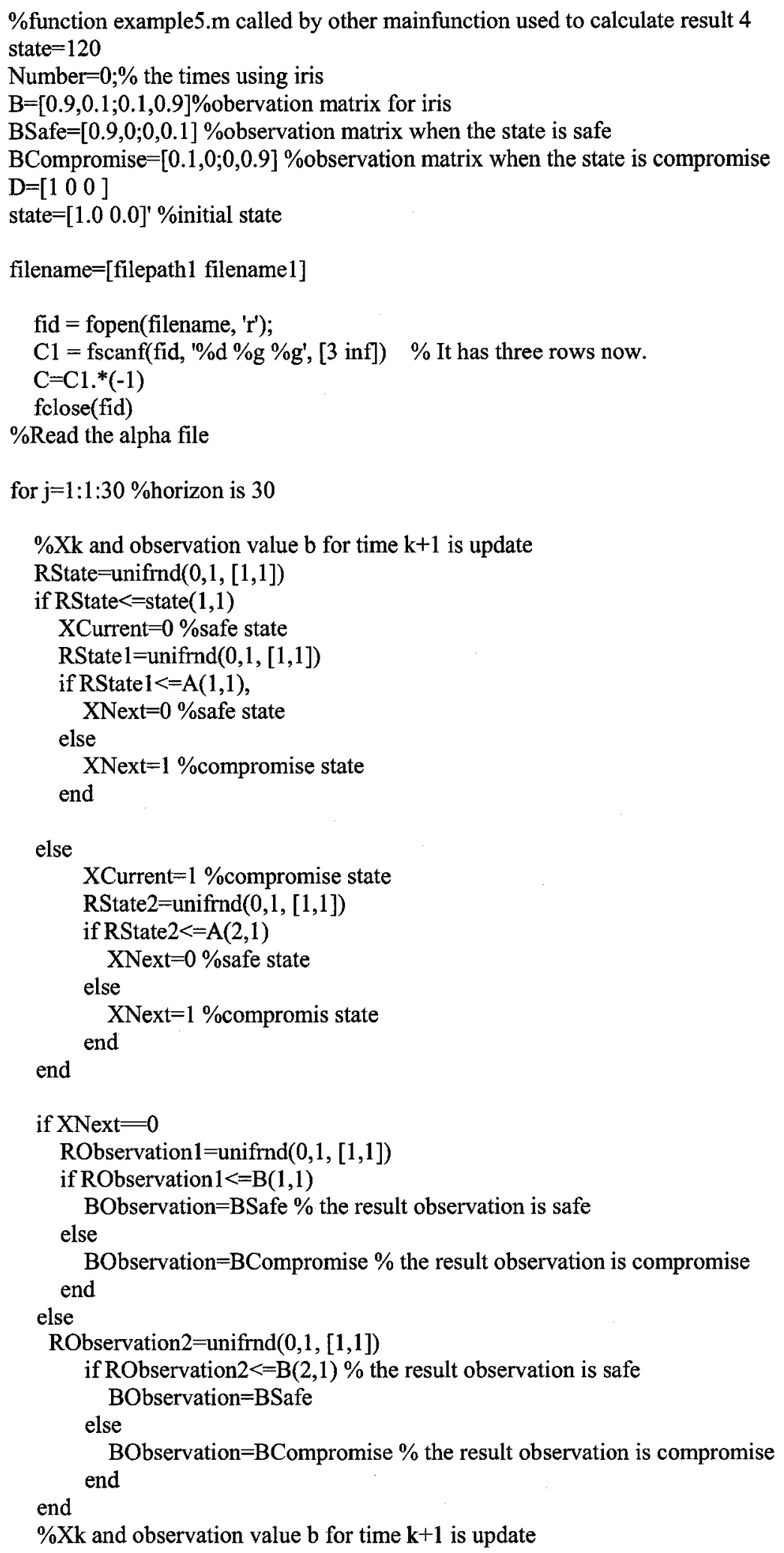

Fig. A-6 Matlab file for computing result 4 page 2 


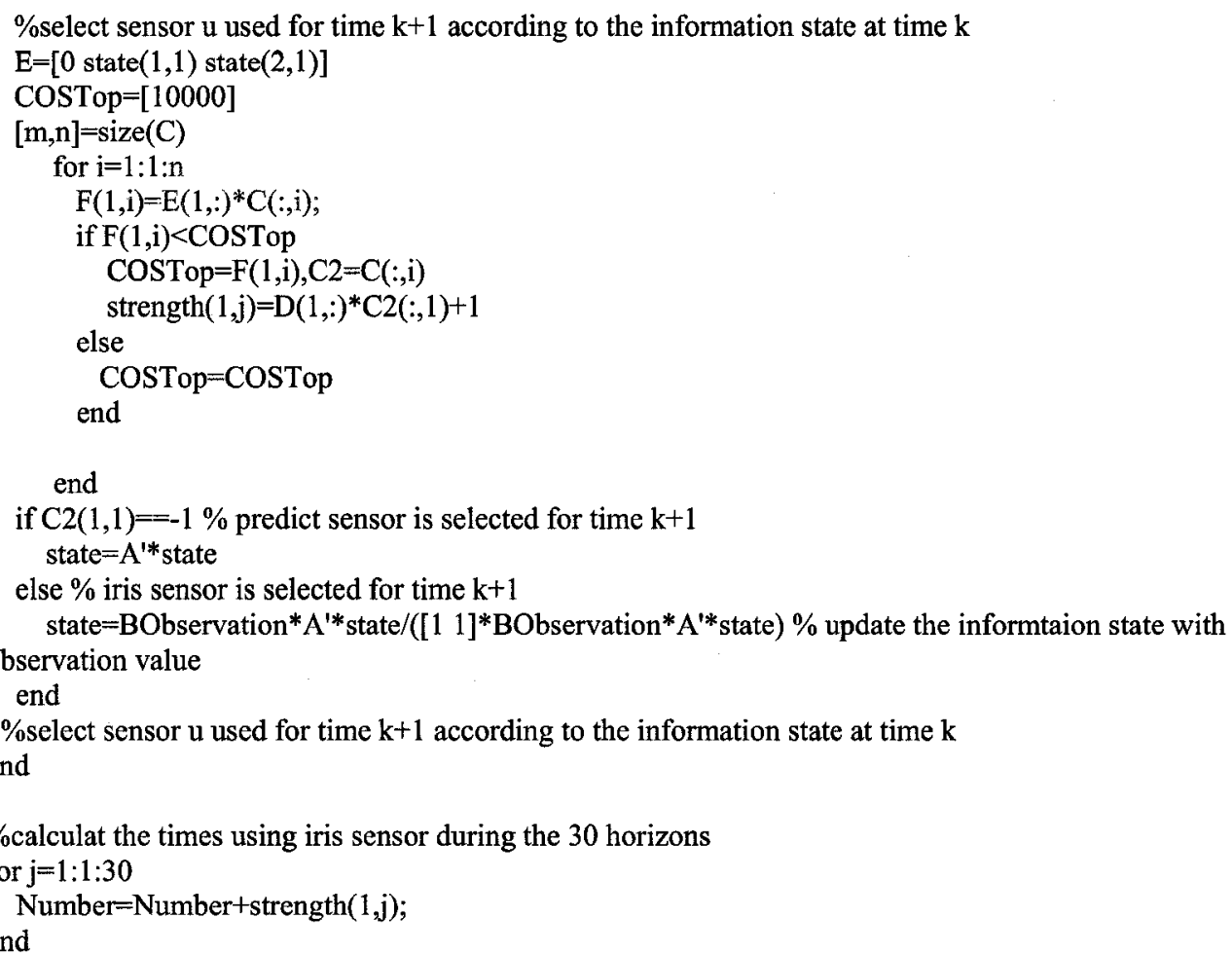

Fig. A-7 Matlab file for computing result 4 page 3 
clear all

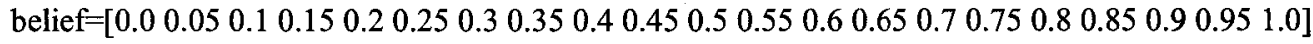

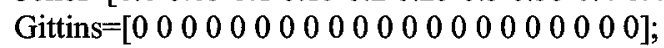

Gittins $1=\left[\begin{array}{lllllllllllllllllllll}0 & 0 & 0 & 0 & 0 & 0 & 0 & 0 & 0 & 0 & 0 & 0 & 0 & 0 & 0 & 0 & 0 & 0 & 0 & 0 & 0\end{array}\right]$

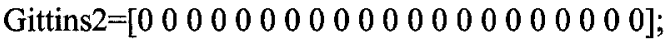

$\%$ Caculate the Gittins index for Server1

fid = fopen('mulbandlsolution4.alpha', 'r'); \% Alpha file stored under the directory of mulband.

$\mathrm{Al}=$ fscanf(fid, ${ }^{\circ} \% \mathrm{~d} \% \mathrm{~g} \% \mathrm{~g} \% \mathrm{~g} \% \mathrm{~g} \% \mathrm{~g} \% \mathrm{~g}$ ', [7 inf]) \% It has seven rows now.

fclose(fid)

$[\mathrm{m} 1, \mathrm{n} 1]=\operatorname{size}(\mathrm{A} 1)$

$\mathrm{n} 1=\mathrm{n} 1-1$

$\mathrm{M} 1=45 \% \mathrm{M}$ bar defined in Equation (4.41) $\mathrm{M} 1=9 /(1-0.8)$

for $\mathrm{i}=1: 21$

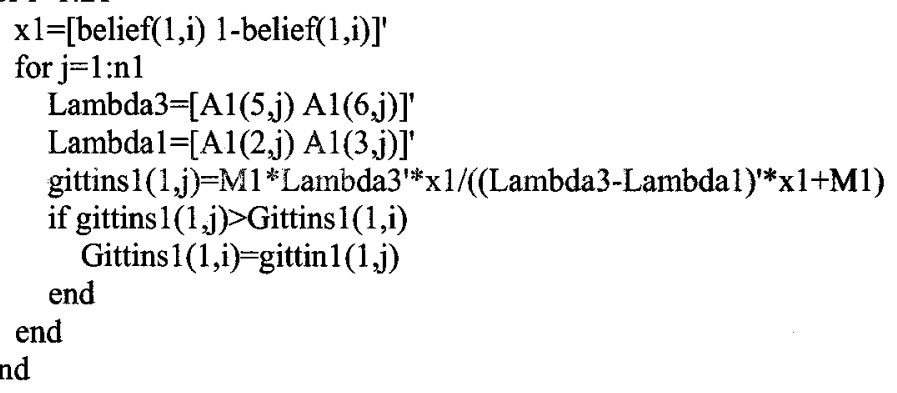

\%Calculate the Gittins index for Server 2

fid = fopen('mulband 'solution7.alpha', 'r');

A3 $=$ fscanf(fid, ${ }^{\circ} \% \mathrm{~d} \% \mathrm{~g} \% \mathrm{~g} \% \mathrm{~g} \% \mathrm{~g} \% \mathrm{~g} \% \mathrm{~g}$ ', [7 inf]) \% It has seven rows now.

fclose(fid)

$[\mathrm{m} 3, \mathrm{n} 3]=\operatorname{size}(\mathrm{A} 3)$

$\mathrm{n} 3=\mathrm{n} 3-1$

$\mathrm{M} 2=37.5 \% \mathrm{M}$ bar defined in Equation (4.41) $\mathrm{M} 1=7.5 /(1-0.8)$

for $\mathrm{i}=1: 21$

$\mathrm{x} 2=[\text { belief }(1, \mathrm{i}) \text { 1-belief }(1, \mathrm{i})]^{\prime}$

for $\mathrm{j}=1: \mathrm{n} 3$

LLLambda3 $=[\mathrm{A} 3(5, \mathrm{j}) \mathrm{A} 3(6, \mathrm{j})]^{\prime}$

LLLambda $1=[A 3(2, j) A 3(3, j)]^{\prime}$

Gittins $2(1, \mathrm{j})=\mathrm{M} 2 *$ LLLambda3 $^{* *} \times 2 /\left((\text { LLLambda3-LLLambda1 })^{\prime *} \times 2+\mathrm{M} 2\right)$

if gittins $2(1, \mathrm{j})>$ Gittins $2(1, \mathrm{i})$

Gittins $2(1, \mathrm{i})=\operatorname{gittin} 2(1, \mathrm{j})$

$$
\text { end }
$$

end

end

\% Plot the Gittins index

plot(belief,Gittin1,'k-.')

hold on

plot(belief,Gittin2,'r-.')

hold off

Xlabel('First component of information state $\left.\mathrm{x}(1)^{\prime}\right)$; ylabel('Gittin Index')

Fig. A-8 Gittins indices for Server type 1 and Server type 2 


\title{
Appendix B: POMDP Files
}

\author{
discount: 0.9 \\ values: reward \\ states: safe compromise \\ actions: iris fingerprint predict \\ observations: accept reject nothing \\ start: 0.80 .2 \\ T: iris \\ 0.850 .15 \\ 0.10 .9 \\ $\mathrm{T}$ : fingerprint \\ 0.850 .15 \\ 0.10 .9 \\ $\mathrm{T}$ : predict \\ 0.850 .15 \\ 0.10 .9 \\ $0:$ iris \\ $\begin{array}{lll}0.95 & 0.05 & 0.0\end{array}$ \\ $\begin{array}{lll}0.05 & 0.95 & 0.0\end{array}$ \\ $\mathrm{O}$ : fingerprint \\ $\begin{array}{lll}0.8 & 0.2 & 0.0\end{array}$ \\ $0.2 \quad 0.80 .0$ \\ $\mathrm{O}$ : predict \\ $\begin{array}{lll}0.0 & 0.0 & 1.0\end{array}$ \\ $\begin{array}{lll}0.0 & 0.0 & 1.0\end{array}$ \\ $\mathrm{R}$ : iris : safe : * : ${ }^{*}-12.0$ \\ $\mathrm{R}$ : iris : compromise : *: * -12.2 \\ $\mathrm{R}$ : fingerprint: safe : * * -8.5 \\ $\mathrm{R}$ : fingerprint: compromise : * : * -12.0 \\ R: predict : safe : * : ${ }^{*}-6.5$ \\ $\mathrm{R}$ : predict : compromise : * * -13.5
}

Fig. B-1 POMDP configuration file of Scenario 2 


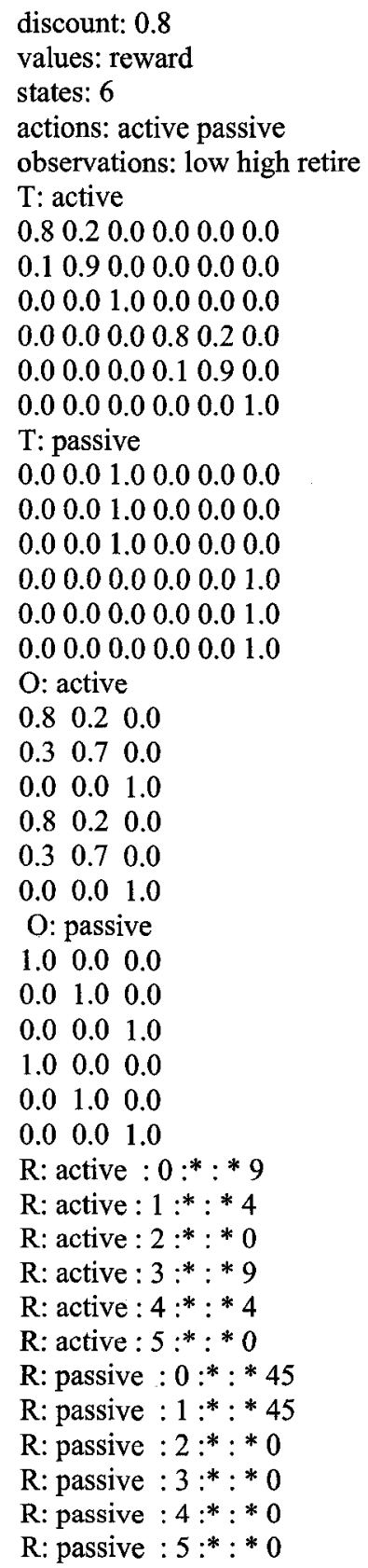

Fig. B-2 POMDP configuration file of Server type 1 


\author{
discount: 0.8 \\ values: reward \\ states: 6 \\ actions: active passive \\ observations: low high retire \\ $\mathrm{T}$ : active \\ 0.850 .150 .00 .00 .00 .0 \\ 0.20 .80 .00 .00 .00 .0 \\ 0.00 .01 .00 .00 .00 .0 \\ 0.00 .00 .00 .850 .150 .0 \\ 0.00 .00 .00 .20 .80 .0 \\ 0.00 .00 .00 .00 .01 .0 \\ $\mathrm{T}$ : passive \\ 0.00 .01 .00 .00 .00 .0 \\ 0.00 .01 .00 .00 .00 .0 \\ 0.00 .01 .00 .00 .00 .0 \\ 0.00 .00 .00 .00 .01 .0 \\ 0.00 .00 .00 .00 .01 .0 \\ 0.00 .00 .00 .00 .01 .0 \\ $\mathrm{O}$ : active \\ $\begin{array}{lll}0.8 & 0.2 & 0.0\end{array}$ \\ $\begin{array}{llll}0.3 & 0.7 & 0.0\end{array}$ \\ $\begin{array}{llll}0.0 & 0.0 & 1.0\end{array}$ \\ $\begin{array}{llll}0.8 & 0.2 & 0.0\end{array}$ \\ $\begin{array}{llll}0.3 & 0.7 & 0.0\end{array}$ \\ $\begin{array}{llll}0.0 & 0.0 & 1.0\end{array}$ \\ 0 : passive \\ $\begin{array}{llll}1.0 & 0.0 & 0.0\end{array}$ \\ $\begin{array}{llll}0.0 & 1.0 & 0.0\end{array}$ \\ $\begin{array}{llll}0.0 & 0.0 & 1.0\end{array}$ \\ $\begin{array}{llll}1.0 & 0.0 & 0.0\end{array}$ \\ $\begin{array}{llll}0.0 & 1.0 & 0.0\end{array}$ \\ $\begin{array}{llll}0.0 & 0.0 & 1.0\end{array}$ \\ $\mathrm{R}$ : active : $0: *$ : * 7.5 \\ $\mathrm{R}$ : active : $1: *: * 5$ \\ $\mathrm{R}$ : active : $2: *: * 0$ \\ $\mathrm{R}$ : active : $3: *: * 7.5$ \\ $\mathrm{R}$ : active : $4: *: * 5$ \\ $\mathrm{R}:$ active : $5: *:^{*} 0$ \\ $\mathrm{R}$ : passive : $0: *:^{*} 37.5$ \\ $\mathrm{R}$ : passive : $1: *$ : 37.5 \\ $\mathrm{R}$ : passive : $2: *: * 0$ \\ $\mathrm{R}$ : passive : $3: *:^{*} 0$ \\ $\mathrm{R}$ : passive : $4:^{*}: * 0$ \\ $\mathrm{R}$ : passive : $5:^{*}: * 0$
}

Fig. B-3 POMDP configuration file of Server type 2 